\title{
Hermite and Laguerre Symmetric Functions Associated with Operators of Calogero-Moser-Sutherland Type
}

\author{
Patrick DESROSIERS ${ }^{\dagger}$ and Martin HALLN $\ddot{A} S^{\ddagger}$ \\ † Instituto Matemática y Física, Universidad de Talca, 2 Norte 685, Talca, Chile \\ E-mail:Patrick.Desrosiers@inst-mat.utalca.cl \\ $\ddagger$ Department of Mathematical Sciences, Loughborough University, \\ Leicestershire, LE11 3TU, UK \\ E-mail: M.A.Hallnas@lboro.ac.uk
}

Received March 22, 2012, in final form July 25, 2012; Published online August 03, 2012

http://dx.doi.org/10.3842/SIGMA.2012.049

\begin{abstract}
We introduce and study natural generalisations of the Hermite and Laguerre polynomials in the ring of symmetric functions as eigenfunctions of infinite-dimensional analogues of partial differential operators of Calogero-Moser-Sutherland (CMS) type. In particular, we obtain generating functions, duality relations, limit transitions from Jacobi symmetric functions, and Pieri formulae, as well as the integrability of the corresponding operators. We also determine all ideals in the ring of symmetric functions that are spanned by either Hermite or Laguerre symmetric functions, and by restriction of the corresponding infinite-dimensional CMS operators onto quotient rings given by such ideals we obtain socalled deformed CMS operators. As a consequence of this restriction procedure, we deduce, in particular, infinite sets of polynomial eigenfunctions, which we shall refer to as super Hermite and super Laguerre polynomials, as well as the integrability, of these deformed CMS operators. We also introduce and study series of a generalised hypergeometric type, in the context of both symmetric functions and 'super' polynomials.
\end{abstract}

Key words: symmetric functions; super-symmetric polynomials; (deformed) CalogeroMoser-Sutherland models

2010 Mathematics Subject Classification: 05E05; 13J05; 81R12

\section{Contents}

1 Introduction $\quad \mathbf{2}$

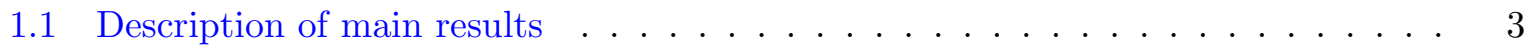

1.2 Notes . . . . . . . . . . . . . . . . . . . . . 4

1.3 Notation and conventions . . . . . . . . . . . . . . . . . 4

2 Symmetric functions $\quad \mathbf{5}$

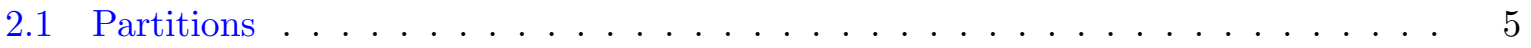

2.2 Symmetric functions . . . . . . . . . . . . . . . . . 6

2.3 Jack's symmetric functions . . . . . . . . . . . . . . . . 7

2.4 CMS operators on the symmetric functions $\ldots \ldots \ldots . \ldots$

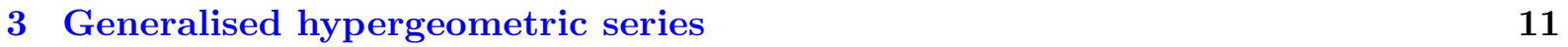

4 Hermite symmetric functions $\quad \mathbf{1 5}$

4.1 A duality relation . . . . . . . . . . . . . . . . . . 16 
4.2 A generating function $\ldots \ldots \ldots \ldots \ldots \ldots \ldots$

4.3 A limit from the Jacobi symmetric functions . . . . . . . . . . . . . . . 21

4.4 Structure of Pieri formulae and invariant ideals . . . . . . . . . . . . . . . . 22

$5 \quad$ Laguerre symmetric functions $\quad \mathbf{2 8}$

5.1 A symmetry property . . . . . . . . . . . . . . . . . 28

5.2 A duality relation . . . . . . . . . . . . . . . . . . . . . . 29

5.3 A generating function . . . . . . . . . . . . . . . . . . . 29

5.4 Limit transition from the Laguerre to the Hermite symmetric functions . . . . . 30

5.5 A limit from the Jacobi symmetric functions . . . . . . . . . . . . . . 30

5.6 Structure of Pieri formulae and invariant ideals . . . . . . . . . . . . . 31

6 Deformed CMS operators and super polynomials 33

6.1 Super Jack polynomials . . . . . . . . . . . . . . . . . . . . . 33

6.2 Deformed CMS operators . . . . . . . . . . . . . . . 36

6.3 Super Hermite polynomials . . . . . . . . . . . . . . . . . . . 38

6.4 Super Laguerre polynomials . . . . . . . . . . . . . . . . . . . . . . . 40

A Differential operators on the symmetric functions 41

B CMS operators on the symmetric functions $\quad 44$

$\begin{array}{ll}\text { C Proof of Theorem } 4.15 & 48\end{array}$

$\begin{array}{ll}\text { References } & 50\end{array}$

\section{Introduction}

The main purpose of this paper is to introduce and study two (non-homogenous) bases for the graded ring of symmetric functions $\Lambda$, which we shall refer to as Hermite and Laguerre symmetric functions due to their relations with the corresponding classical orthogonal polynomials.

One of the main themes in the theory of symmetric functions is the description of various homogeneous bases for $\Lambda$. Important examples include the Schur symmetric functions and, more generally, Jack's and Macdonald's symmetric functions, which are one- and twoparameter deformations thereof; see, e.g., Macdonald [29]. These symmetric functions arise as (inverse) limits with respect to the number of variables $n$ of corresponding symmetric polynomials.

In the early 1990s, Lassalle [24, 25, 26] and Macdonald [28] independently introduced natural $n$-variable generalisations of the classical orthogonal polynomials (of Hermite, Laguerre and Jacobi), depending on one 'extra' parameter $\alpha .{ }^{1}$ For the special value $\alpha=2$ they are naturally realised as functions on the $n \times n$ real symmetric matrices, and as such had been introduced already in work of James [16] in the Hermite, Herz [14] and Constantine [8] in the Laguerre, and James and Constantine [17] in the Jacobi case; see also Muirhead [31]. In contrast to the Schur polynomials, these $n$-variable generalisations of the classical orthogonal polynomials do not posses limits as $n$ goes to infinity.

We shall circumvent this problem by introducing an additional (formal) parameter $p_{0}$, which can be viewed as a zeroth-order power sum symmetric function, and consider symmetric functions over the field $\mathbb{Q}\left(p_{0}\right)$. The Hermite and Laguerre symmetric functions are then the unique

\footnotetext{
${ }^{1}$ The generalised Jacobi polynomials were previously introduced by Debiard [9], and independently by Heckman and Opdam [13] (see also [36]). However, the authors in [9, 13] do not explicitly make use of Jack polynomial theory, which contrasts with the approach of Lassalle and Macdonald [25, 28].
} 
elements in $\Lambda$ such that if we set $p_{0}=n \in \mathbb{N}$, and restrict to $\Lambda_{n}$, the ring of symmetric polynomials in $n$ variables, then we recover the corresponding $n$-variable polynomials of Lassalle and Macdonald.

\subsection{Description of main results}

1. The Hermite and Laguerre symmetric functions will be indexed by partitions, and defined as eigenfunctions of certain infinite-dimensional analogues of partial differential operators of Calogero-Moser-Sutherland (CMS) type; see Definitions 4.1 and 5.1. When restricted to a finite number of variables, they reduce to precisely such partial differential operators, a fact that directly yields the relation to the multivariable Hermite and Laguerre polynomials introduced by Lassalle and Macdonald.

2. In Propositions 4.5 and 5.4 we establish particular duality relations, which are not present at the level of symmetric polynomials. For Jack's symmetric functions the corresponding duality is well-known, and amounts to taking the conjugate of the labelling partition and sending $\alpha \rightarrow$ $1 / \alpha$; see Section VI.10 in Macdonald [29].

3. We show that a number of well-known and important results on, and properties of, the $n$-variable Hermite and Laguerre polynomials generalise in a straightforward manner to the symmetric functions setting. These include generating functions, limit transitions from Jacobi symmetric functions, recently introduced by Sergeev and Veselov [41], as well as higher-order eigenoperators.

4. We also establish Pieri formulae: the expansion of products of algebraic generators and linear basis elements of $\Lambda$ in the same basis elements. They are exhibited in Propositions 4.18 and 5.8. In this case, the algebraic generators are the elementary symmetric functions. In the $n$-variable case such formulae were obtained by van Diejen [46]. However, his formulae do not directly lift to the level of symmetric functions, since they depend in a non-trivial manner on $n$.

5. These Pieri formulae allow us to completely describe the set of ideals in $\Lambda$ that are invariant under the full algebra of eigenoperators of either the Hermite or the Laguerre symmetric functions. In the Hermite case we show that such an ideal exists only if we set $p_{0}=n-\alpha m$ for some $n, m \in \mathbb{N}_{0} \equiv \mathbb{N} \cup\{0\}$. In that case the ideal is unique, and we give a basis in terms of Hermite symmetric functions; see Theorem 4.22. In the Laguerre case such an ideal exists also for $p_{0}=n+1-\alpha(m+a+1)$, which again is unique, and we provide a basis in terms of Laguerre symmetric functions; see Theorem 5.11.

6 . The restriction of a differential operator on $\Lambda$ onto a given quotient ring $\Lambda / I$ is possible if and only if the ideal $I$ is invariant under the operator in question. For $p_{0}=n-\alpha m$, we give an explicit realisation of the restrictions of the eigenoperators of both the Hermite and the Laguerre symmetric functions onto the corresponding quotient ring. More precisely, in Sections 6.3 and 6.4 we show that they are given by particular partial differential operators of so-called deformed CMS type. The operators in question were previously considered by Feigin [11], Hallnäs and Langman [12]. They also appeared, in a disguised form, in the work of Guhr and Kohler [21], and more recently in the context of Random Matrix Theory [10]. For $n=0$ or $m=0$ these operators reduce to ordinary CMS operators.

7. Through this restriction procedure our previous results in the paper immediately yield corresponding results for these deformed CMS operators. In particular, the Hermite and Laguerre symmetric functions restrict to corresponding eigenfunctions that are polynomials, which, following previous results in the literature, we shall refer to as super Hermite and super Laguerre polynomials, respectively.

8. In order to establish a wider context for our results, we also discuss the notion of differential operators on the ring of symmetric functions in Appendix A. In particular, we establish an 
explicit description of such operators in terms of their action on power sum symmetric functions. This will make it clear that the infinite-dimensional CMS operators mentioned above are indeed differential operators on the symmetric functions. In Appendix B we isolate certain results that hold true not only in the Hermite and Laguerre cases. This includes the fact that a generic infinite-dimensional CMS operator of second order has a complete set of eigenfunctions in the ring of symmetric functions.

One of our main motivations for this paper, and perhaps its most important consequence, is that lifting multivariable Hermite and Laguerre polynomials to the level of symmetric functions unifies the CMS operators in question with their corresponding deformed analogues, as mentioned under item (6). In particular, this provides a conceptual understanding of the latter operators, and many of their key properties are thus inherited from the undeformed case. A further appraisal of this point of view can be found in a paper by Sergeev and Veselov [44].

\subsection{Notes}

The trick of lifting a family of (non-stable) symmetric polynomials to symmetric functions by introducing an additional formal parameter, which represents the dependence on the number of variables $n$, has previously been used by Rains [38] and Sergeev and Veselov [41]. Rains deals with the Koornwinder polynomials, whereas Sergeev and Veselov consider Jacobi symmetric polynomials.

In addition, this trick was recently used by Olshanski [35] (see also [34]) to introduce Laguerre and Meixner symmetric functions. His notion of Laguerre symmetric functions is a special case of ours, corresponding to $\alpha=1$. There is certain overlap between Olshanski's paper and the present one, but they are to a large extent complementary. Indeed, he addresses a number of problems that are not considered here. For example, orthogonality of the Laguerre symmetric functions, as well as a corresponding infinite-dimensional diffusion process. On the other hand, Olshanski does not discuss the Hermite case, the classification of invariant ideals in the ring of symmetric functions, and 'super' polynomials.

The generalised Hermite and Laguerre polynomials in $n$-variables are, up to an overall (groundstate) factor, eigenfunctions of Schrödinger operators that define integrable quantum $n$-body systems. For the Hermite case the corresponding system is essentially the one originally considered by Calogero [5], and the system corresponding to the Laguerre polynomials appear in Section 11 of Olshanetsky and Perelomov [33], as a generalisation of Calogero's system corresponding to the root system $B_{n}$. A detailed discussion of this relationship between multivariable Hermite and Laguerre polynomials on the one hand and integrable quantum many-body systems on the other can be found in Baker and Forrester [2] and van Diejen [46]. These papers also contain a number of important results on such polynomials, as well as references to further related results in the literature.

\subsection{Notation and conventions}

We conclude this introduction with a few remarks on notation. In particular, on the parameter $\alpha$ : in the context of integrable systems it is typically replaced by its inverse $1 / \alpha$, denoted by a number of different letters, e.g., $-k$ in $[41,42]$ or $\theta$ in $[19,32,43]$; and in literature related to Random Matrix Theory $\beta=2 / \alpha$ is often used (as, e.g., in Baker and Forrester [2]). In an attempt to minimise confusion we shall throughout this paper only make use of the parameter $\alpha$. Regarding the natural numbers, we shall require both the set including and the set excluding the element zero. For that reason, we make use of the conventions $\mathbb{N}_{0} \equiv\{0,1,2, \ldots\}$ and $\mathbb{N} \equiv\{1,2, \ldots\}$. 


\section{Symmetric functions}

This section is largely a brief review of definitions and results from the theory of symmetric functions that we shall make use of. This review is intended to serve two purposes: firstly, to fix our notation, and secondly we hope that it will make the paper accessible to a somewhat wider audience. Throughout this section we shall in most cases adhere to the notation in Macdonald's book [29], to which the reader is referred for further details.

\subsection{Partitions}

A partition $\lambda=\left(\lambda_{1}, \lambda_{2}, \ldots, \lambda_{i}, \ldots\right)$ is a sequence of non-negative integers $\lambda_{i}$ such that

$$
\lambda_{1} \geq \lambda_{2} \geq \cdots \geq \lambda_{i} \geq \cdots
$$

and only a finite number of the terms $\lambda_{i}$ are non-zero. The number of non-zero terms is referred to as the length of $\lambda$, and is denoted $\ell(\lambda)$. We shall not distinguish between two partitions that differ only by a string of zeros. The weight of a partition $\lambda$ is the sum

$$
|\lambda|:=\lambda_{1}+\lambda_{2}+\cdots
$$

of its parts, and its diagram is the set of points $(i, j) \in \mathbb{N}^{2}$ such that $1 \leq j \leq \lambda_{i}$. Reflection in the diagonal produces the conjugate partition $\lambda^{\prime}=\left(\lambda_{1}^{\prime}, \lambda_{2}^{\prime}, \ldots\right)$. We use the notation $e_{i}, i \in \mathbb{N}$, for the sequence defined by $\left(e_{i}\right)_{j}=\delta_{i j}$, where $\delta_{i j}$ is the Kronecker delta. In addition, we shall make use of the notation

$$
\lambda^{(i)}=\lambda+e_{i}, \quad \lambda_{(i)}=\lambda-e_{i} .
$$

The set of all partitions of a given weight are partially ordered by the dominance order: $\lambda \leq \mu$ if and only if $\sum_{i=1}^{k} \lambda_{i} \leq \sum_{i=1}^{k} \mu_{i}$ for all $k \in \mathbb{N}$. One easily verifies that $\lambda \leq \mu$ if and only if $\mu^{\prime} \leq \lambda^{\prime}$. We shall also require the inclusion order on the set of all partitions, defined by $\mu \subseteq \lambda$ if and only if $\lambda_{i} \leq \mu_{i}$ for all $i$, or equivalently, if and only if the diagram of $\mu$ is contained in that of $\lambda$.

To a partition $\lambda$ is associated the following product of $\alpha$-deformed hook lengths:

$$
h_{\lambda}=\prod_{(i, j) \in \lambda}\left(1+a_{\lambda}(i, j)+\frac{1}{\alpha} l_{\lambda}(i, j)\right),
$$

involving the arm-lengths and leg-lengths

$$
a_{\lambda}(i, j)=\lambda_{i}-j, \quad l_{\lambda}(i, j)=\lambda_{j}^{\prime}-i .
$$

Closely related is the following $\alpha$-deformation of the Pochhammer symbol:

$$
[x]_{\lambda}=\prod_{1 \leq i \leq \ell(\lambda)}\left(x-\frac{i-1}{\alpha}\right)_{\lambda_{i}}=\prod_{(i, j) \in \lambda}\left(x+a_{\lambda}^{\prime}(i, j)-\frac{1}{\alpha} l_{\lambda}^{\prime}(i, j)\right)
$$

with $(x)_{n} \equiv x(x+1) \cdots(x+n-1)$ the ordinary Pochhammer symbol, to which $[x]_{\lambda}$ clearly reduces for $\ell(\lambda)=1$, and where the second expression for $[x]_{\lambda}$ involves the co-arm-lengths and co-leg-lengths

$$
a_{\lambda}^{\prime}(i, j)=j-1, \quad l_{\lambda}^{\prime}(i, j)=i-1 .
$$




\subsection{Symmetric functions}

The ring of symmetric polynomials in $n$ indeterminants $x=\left(x_{1}, \ldots, x_{n}\right)$ with integer coefficients,

$$
\Lambda_{n}=\mathbb{Z}\left[x_{1}, \ldots, x_{n}\right]^{S_{n}},
$$

has a natural grading given by the degree of the polynomials:

$$
\Lambda_{n}=\bigoplus_{k \geq 0} \Lambda_{n}^{k}
$$

where $\Lambda_{n}^{k}$ is the submodule consisting of all homogeneous symmetric polynomials of degree $k$.

For a given $k$ and $n \in \mathbb{N}$, consider the homomorphism $\rho_{n, n-1}^{k}: \Lambda_{n}^{k} \rightarrow \Lambda_{n-1}^{k}$ defined by

$$
\left(\rho_{n, n-1}^{k} f\right)\left(x_{1}, \ldots, x_{n-1}\right)=f\left(x_{1}, \ldots, x_{n-1}, 0\right) .
$$

Let $\Lambda^{k}$ denote the module consisting of all sequences $\left(f_{1}, f_{2}, \ldots, f_{n}, \ldots\right)$ such that $f_{n} \in \Lambda_{n}^{k}$ and $\rho_{n, n-1}^{k} f_{n}=f_{n-1}$, and with the module structure given by term wise operations. The ring of symmetric functions can then be defined as the graded ring

$$
\Lambda=\bigoplus_{k \geq 0} \Lambda^{k}
$$

We note the restriction homomorphisms $\rho_{n}^{k}: \Lambda^{k} \rightarrow \Lambda_{n}^{k}$, which sends $f \in \Lambda^{k}$ to $f_{n}$, and $\rho_{n} \equiv$ $\oplus_{k \geq 1} \rho_{n}^{k}: \Lambda \rightarrow \Lambda_{n}$.

Given a (commutative) ring $A$ we will use the notation $\Lambda_{A}$ for the tensor product $A \otimes_{\mathbb{Z}} \Lambda$, and similarly for $\Lambda_{n}$. In this paper we shall mainly be concerned with either the fields

$$
\mathbb{F}=\mathbb{Q}(\alpha), \quad \mathbb{K}=\mathbb{Q}(a, \alpha),
$$

or the corresponding extensions generated by the indeterminate $p_{0}$,

$$
\mathbf{F}=\mathbb{F}\left(p_{0}\right), \quad \mathbf{K}=\mathbb{K}\left(p_{0}\right) .
$$

There are several important and useful generators of $\Lambda$. We shall make use of the elementaryand power sum symmetric functions, given by

$$
\begin{array}{ll}
e_{r}:=\lim _{\leftarrow} e_{r}(x), \quad e_{r}(x)=\sum_{1 \leq i_{1}<\cdots<i_{r} \leq n} x_{i_{1}} \cdots x_{i_{r}}, \\
p_{r}:=\lim _{\leftarrow} p_{r}(x), \quad p_{r}(x)=\sum_{i=1}^{n} x_{i}^{r},
\end{array}
$$

respectively. Here, $\lim _{\leftarrow} g_{r}$, where $g_{r}\left(x_{1}, \ldots, x_{n}\right) \in \Lambda_{n}^{r}$ for all $n \in \mathbb{N}$, denotes the sequence $\left(g_{r}\left(x_{1}\right), g_{r}\left(x_{1}, x_{2}\right), g_{r}\left(x_{1}, x_{2}, x_{3}\right), \ldots\right) \in \Lambda^{r}$, and $r$ is allowed to be any non-negative integer. To be precise, the $e_{r}$ generate $\Lambda$, and are algebraically independent over $\mathbb{Z}$; while, on the other hand, the $p_{r}$ generate $\Lambda_{\mathbb{Q}}$, but not $\Lambda$, and are algebraically independent over $\mathbb{Q}$. We recall the standard notation

$$
e_{\lambda}=e_{\lambda_{1}} e_{\lambda_{2}} \cdots, \quad p_{\lambda}=p_{\lambda_{1}} p_{\lambda_{2}} \cdots,
$$

where $\lambda$ is any partition. In addition, we shall make use of the monomial symmetric functions

$$
m_{\lambda}:=\lim _{\leftarrow} m_{\lambda}(x), \quad m_{\lambda}(x)=\sum_{P} x_{1}^{\lambda_{P(1)}} \cdots x_{n}^{\lambda_{P(n)}},
$$

where the sum extends over all distinct permutations $P$ of $\lambda$. As $\lambda$ runs through all partitions, the $e_{\lambda}$ and $m_{\lambda}$ form a basis for $\Lambda$ and the $p_{\lambda}$ for $\Lambda_{\mathbb{Q}}$. 


\subsection{Jack's symmetric functions}

Jack's symmetric functions form a further important, albeit more intricate, basis for $\Lambda_{\mathbb{F}}$, which will be a key ingredient in many constructions in this paper. In order to recall their definition we start from the CMS operator

$$
D_{n}=\sum_{i=1}^{n} x_{i}^{2} \frac{\partial^{2}}{\partial x_{i}^{2}}+\frac{2}{\alpha} \sum_{i \neq j} \frac{x_{i} x_{j}}{x_{i}-x_{j}} \frac{\partial}{\partial x_{i}} .
$$

It is important to note that this operator preserves $\Lambda_{\mathbb{F}, n}$. This follows from invariance under permutations of $x$ and the observation that, for $p \in \Lambda_{\mathbb{F}, n}$, the polynomial $\left(\partial / \partial x_{i}-\partial / \partial x_{j}\right) p$ is antisymmetric under the interchange of $x_{i}$ and $x_{j}$, and hence divisible by $x_{i}-x_{j}$. Moreover, it is stable with respect to $\rho_{n, n-1}$ :

$$
\rho_{n, n-1} \circ D_{n}=D_{n-1} \circ \rho_{n, n-1} .
$$

Hence, there is a unique operator $D$ on $\Lambda_{\mathbb{F}}$ such that $\rho_{n} \circ D=D_{n} \circ \rho_{n}$, given by the sequence $\left(D_{1}, D_{2}, \ldots, D_{n}, \ldots\right)$. Now, for a partition $\lambda$, the (monic) Jack's symmetric function $P_{\lambda}$ is the unique eigenfunction of this operator of the form

$$
P_{\lambda}=m_{\lambda}+\sum_{\mu<\lambda} c_{\lambda \mu} m_{\mu}, \quad c_{\lambda \mu} \in \mathbb{F}
$$

Note that $P_{\lambda}$ is homogeneous of degree $|\lambda|$. For further details see, e.g., Chapter VI of Macdonald [29].

It is a remarkable fact that to each so-called shifted symmetric function corresponds an eigenoperator of Jack's symmetric functions. We recall that the algebra of shifted symmetric polynomials $\Lambda_{\alpha, n} \equiv \Lambda_{\mathbb{F}, \alpha, n}$ consists of all polynomials $p\left(x_{1}, \ldots, x_{n}\right)$ (over the field $\mathbb{F} \equiv \mathbb{Q}(\alpha)$ ) that are symmetric in the shifted variables $x_{i}-i / \alpha$; see, e.g., Okounkov and Olshanski [32]. It has a natural filtration, given by the degree of the polynomials:

$$
\Lambda_{\alpha, n}^{0} \subset \Lambda_{\alpha, n}^{1} \subset \cdots \subset \Lambda_{\alpha, n}^{k} \subset \cdots,
$$

where $\Lambda_{\alpha, n}^{k}$ is the subspace of polynomials of degree at most $k$. As in the construction of the ring of symmetric functions, we can introduce linear spaces $\Lambda_{\alpha}^{k}$, and the algebra of shifted symmetric functions is then given as the filtered algebra

$$
\Lambda_{\alpha}=\bigcup_{k \geq 0} \Lambda_{\alpha}^{k}
$$

In particular, this algebra is freely generated by the shifted power sums

$$
\pi_{r, \alpha}:=\lim _{\leftarrow} \pi_{r, \alpha}(x), \quad \pi_{r, \alpha}(x)=\sum_{i=1}^{n}\left(\left(x_{i}-\frac{i}{\alpha}\right)^{r}-\left(-\frac{i}{\alpha}\right)^{r}\right) .
$$

Now, for any $f \in \Lambda_{\alpha}$, there is a unique operator $\mathcal{L}_{f}$ on $\Lambda_{\mathbb{F}}$ such that

$$
\mathcal{L}_{f} P_{\lambda}=f(\lambda) P_{\lambda}
$$

for all partitions $\lambda$. In particular, the operator $D$ can be obtained as a linear combination of $\mathcal{L}_{\pi_{1, \alpha}}$ and $\mathcal{L}_{\pi_{2, \alpha}}$. A construction of these operators using Cherednik-Dunkl operators [4, 7, 37] can be found in Section 4 of Sergeev and Veselov [43]. 
We recall the natural analogue of the specialisation $\left(x_{1}, \ldots, x_{n}\right)=(1, \ldots, 1)$ : for any $X \in \mathbb{F}$ define a homomorphism $\epsilon_{X}: \Lambda_{\mathbb{F}} \rightarrow \mathbb{F}$ by setting

$$
\epsilon_{X}\left(p_{r}\right)=X, \quad r \in \mathbb{N} .
$$

Stanley [45] (see also Section VI.10 in Macdonald [29]) has shown that the corresponding specialisation of Jack's symmetric functions is given by

$$
\epsilon_{X}\left(P_{\lambda}\right)=\prod_{(i, j) \in \lambda} \frac{X+\alpha a_{\lambda}^{\prime}(i, j)-l_{\lambda}^{\prime}(i, j)}{\alpha a_{\lambda}(i, j)+l_{\lambda}(i, j)+1},
$$

c.f. $(2.2)$ and (2.4).

\subsection{CMS operators on the symmetric functions}

The $n$-variable Hermite and Laguerre polynomials introduced by Lassalle and Macdonald can be defined as eigenfunctions of CMS operators of the form

$$
\mathcal{L}_{n}=\sum_{k=0}^{2} a_{k} D_{n}^{k}+\sum_{\ell=0}^{1} b_{\ell} E_{n}^{\ell}
$$

for some choice of coefficients $a_{k}$ and $b_{\ell}$, and where the 'building blocks'

$$
E_{n}^{\ell}=\sum_{i=1}^{n} x_{i}^{\ell} \frac{\partial}{\partial x_{i}}
$$

and

$$
D_{n}^{k}=\sum_{i=1}^{n} x_{i}^{k} \frac{\partial^{2}}{\partial x_{i}^{2}}+\frac{2}{\alpha} \sum_{i \neq j} \frac{x_{i}^{k}}{x_{i}-x_{j}} \frac{\partial}{\partial x_{i}} .
$$

More specifically, the Hermite and Laguerre cases correspond to the following choice of coefficients:

$$
\begin{array}{llrl}
\left(a_{2}, a_{1}, a_{0}\right)=(0,0,1), & \left(b_{1}, b_{0}\right)=(-2,0), & \text { Hermite } \\
\left(a_{2}, a_{1}, a_{0}\right)=(0,1,0), & \left(b_{1}, b_{0}\right)=(-1, a+1), & \text { Laguerre. }
\end{array}
$$

A generic operator $\mathcal{L}_{n}$ is not stable under restrictions of the number of variables, i.e., $\rho_{n, n-1} \circ \mathcal{L}_{n} \neq$ $\mathcal{L}_{n-1} \circ \rho_{n, n-1}$. Consequently, it does not directly lift to an operator on $\Lambda_{\mathbb{F}}$. In fact, only among the eigenoperators for the Jack polynomials can such stable CMS operators be found.

Nevertheless, by introducing a new indeterminate $p_{0}$, which effectively encodes the dependence on the number of variables $n$, to each CMS operator $\mathcal{L}_{n}$ we can in a natural manner assign an operator $\mathcal{L}$ on $\Lambda_{\mathbb{F}}$. In the Jacobi case $\left(\left(a_{2}, a_{1}, a_{0}\right)=(1,2,0)\right.$ and $\left(b_{1}, b_{0}\right)=$ $(-p-2 q+1,-2 p-2 q+1))$ this fact was demonstrated by Sergeev and Veselov [41]. Closely related is an earlier paper by Rains [38], which concerns a symmetric function analogue of the Koornwinder polynomials.

As a first step towards making these remarks precise, we shall rewrite the CMS operators (2.12), and (2.13) in a more convenient form. Fix $n \in \mathbb{N}$ and let $r=1, \ldots, n$. Then, we can define a differential operator $\partial^{(n)}\left(p_{r}\right)$ on $\Lambda_{\mathbb{F}, n}$ by requiring that $\partial^{(n)}\left(p_{r}\right) 1=0$ and

$$
\partial^{(n)}\left(p_{r}\right) p_{s}= \begin{cases}1, & r=s \\ 0, & r \neq s\end{cases}
$$

for $s=1, \ldots, n$. 
Lemma 2.1. Set $p_{0}=n$. Then, the differential operators $E_{n}^{\ell}$ and $D_{n}^{k}$ are given by

$$
E_{n}^{\ell}=\sum_{r=1}^{n} r p_{r+\ell-1} \partial^{(n)}\left(p_{r}\right)
$$

and

$$
\begin{aligned}
D_{n}^{k}= & \sum_{r, q=1}^{n} r q p_{r+q+k-2} \partial^{(n)}\left(p_{r}\right) \partial^{(n)}\left(p_{q}\right)+\sum_{r=2}^{n} r(r-1) p_{r+k-2} \partial^{(n)}\left(p_{r}\right) \\
& +\frac{1}{\alpha} \sum_{r=1}^{n} r \sum_{m=0}^{r+k-2}\left(p_{r+k-2-m} p_{m}-p_{r+k-2}\right) \partial^{(n)}\left(p_{r}\right),
\end{aligned}
$$

respectively.

Proof. We recall that $E_{n}^{\ell}$ are first-order differential operators, and that $\Lambda_{\mathbb{F}, n}$ is generated by the power sums $p_{r}(x)$ with $r=1, \ldots, n$. Hence, it is sufficient to compute their action on said power sums. This yields (2.14a).

We thus turn to the differential operators $D_{n}^{k}$, and observe that their first-order terms act on the power sums as follows:

$$
\begin{aligned}
2 \sum_{i \neq j} \frac{x_{i}^{k}}{x_{i}-x_{j}} \frac{\partial}{\partial x_{i}} p_{r}(x) & =\sum_{i \neq j} \frac{1}{x_{i}-x_{j}}\left(x_{i}^{k} \frac{\partial}{\partial x_{i}}-x_{j}^{k} \frac{\partial}{\partial x_{j}}\right) p_{r}(x)=\sum_{i \neq j} r \sum_{m=0}^{r+k-2} x_{i}^{r+k-m-2} x_{j}^{m} \\
& =r \sum_{m=0}^{r+k-2}\left(p_{r+k-2-m}(x) p_{m}(x)-p_{r+k-2}(x)\right) .
\end{aligned}
$$

For the second-order terms, it sufficient to know the action on the power sums $p_{r}$ and the products of two power sums, i.e., on the terms $p_{r} p_{q}$ with $r, q=1, \ldots, n$. If we allow $r, q=0$ and set $p_{r}(x) \equiv 0$ for $r<0$, then these cases are all included in the formula

$$
\begin{aligned}
\sum_{i=1}^{n} x_{i}^{k} \frac{\partial^{2}}{\partial x_{i}^{2}} p_{r}(x) p_{q}(x)= & r(r-1) p_{r+k-2}(x) p_{q}(x)+2 r q p_{r+q+k-2}(x) \\
& +q(q-1) p_{r}(x) p_{q+k-2}(x) .
\end{aligned}
$$

Combining these facts we obtain (2.14b).

Remark 2.2. These expressions for the differential operators $E_{n}^{\ell}$ and $D_{n}^{k}$ involve power sums $p_{r}\left(x_{1}, \ldots, x_{n}\right)$ with $r>n$. In principle, such terms can be rewritten in terms of power sums with $r \leq n$. However, we have refrained from doing so since this would lead to rather complicated expressions. In addition, we are ultimately interested in operators on the algebra of symmetric functions, and there are no non-trivial relations between the power sum symmetric functions $p_{r}$.

We now let $p_{0}$ be an indeterminate, and consider the field $\mathbf{F} \equiv \mathbb{F}\left(p_{0}\right)$. It is clear that we can not specialise all $f \in \mathbf{F}$ to $p_{0}=n$. Indeed, this is possible if and only if $f \in \mathbf{F}_{\left(p_{0}-n\right)}$ : the (local) algebra of rational functions $g / h$ in $p_{0}$ over $\mathbb{F}$ such that $h(n) \neq 0$. For simplicity of exposition, we shall make use of the short-hand notation $\mathbf{F}^{(n)} \equiv \mathbf{F}_{\left(p_{0}-n\right)}$. We can now introduce, for each $n \in \mathbb{N}$, the specialisation map $\phi_{n}: \mathbf{F}^{(n)} \rightarrow \mathbb{F}$ by setting

$$
\phi_{n}(f)=\left.f\right|_{p_{0}=n},
$$


and thereby the homomorphism $\varphi_{n}: \Lambda_{\mathbf{F}^{(n)}} \rightarrow \Lambda_{\mathbb{F}, n}$ by

$$
\varphi_{n}(f \otimes p)=\phi_{n}(f) \otimes \rho_{n}(p) .
$$

We note that $\varphi_{n}$ is surjective for all $n \in \mathbb{N}$.

On $\Lambda_{\mathbf{F}}$ we have obvious analogues $\partial\left(p_{r}\right)$ of the differential operators $\partial^{(n)}\left(p_{r}\right)$; see Appendix A. Moreover, in a natural sense, the former differential operators are of degree $-r$; see the discussion preceding Lemma A.2. Lemma 2.1 thus suggests the following definition of differential operators $E^{\ell}$ and $D^{k}$ on $\Lambda_{\mathbf{F}}$ :

Definition 2.3. Let $\ell, k \in \mathbb{N}_{0}$. We then define differential operators $E^{\ell}$ and $D^{k}$ on $\Lambda_{\mathbf{F}}$ by

$$
E^{\ell}=\sum_{r=1}^{\infty} r p_{r+\ell-1} \partial\left(p_{r}\right)
$$

and

$$
\begin{aligned}
D^{k}= & \sum_{r, q=1}^{\infty} r q p_{r+q+k-2} \partial\left(p_{r}\right) \partial\left(p_{q}\right)+\sum_{r=2}^{\infty} r(r-1) p_{r+k-2} \partial\left(p_{r}\right) \\
& +\frac{1}{\alpha} \sum_{r=1}^{\infty} r \sum_{m=0}^{r+k-2}\left(p_{r+k-2-m} p_{m}-p_{r+k-2}\right) \partial\left(p_{r}\right),
\end{aligned}
$$

respectively.

That this is a natural definition is confirmed by the following lemma:

Lemma 2.4. Fix $k, \ell \in \mathbb{N}_{0}$. Then, $E^{\ell}$ and $D^{k}$ are homogeneous differential operators on $\Lambda_{\mathbf{F}}$ of degree $\ell-1$ and $k-2$, respectively. Moreover, they are the unique operators on $\Lambda_{\mathbf{F}}$ such that the diagrams

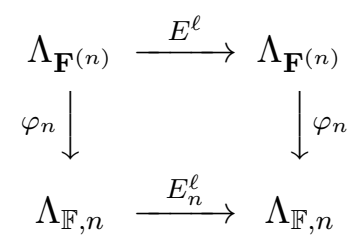

and

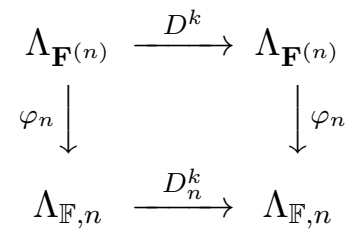

are commutative for all $n \in \mathbb{N}$.

Proof. The fact that both $E^{\ell}$ and $D^{k}$ are differential operators on $\Lambda_{\mathbf{F}}$ is a direct consequence of Proposition A.1. The stated homogeneity and degrees of $E^{\ell}$ and $D^{k}$ follows immediately from the observation that $\partial\left(p_{r}\right)$ and $p_{r}$ are homogeneous of degree $-r$ and $r$, respectively.

It follows from Definition 2.3 and (2.12) that

$$
\varphi_{n}\left(E^{\ell} p_{r}\right)=r p_{r+\ell-1}\left(x_{1}, \ldots, x_{n}\right)=E_{n}^{\ell}\left(\varphi_{n} p_{r}\right), \quad r \in \mathbb{N},
$$

where $p_{0}\left(x_{1}, \ldots, x_{n}\right) \equiv n$. We note that $E^{\ell} p_{0}=0$. Since $E^{\ell}$ and $E_{n}^{\ell}$ are first-order differential operators, and $\varphi_{n}$ a $\mathbb{F}$-algebra homomorphism, this implies (2.17a). We observe that (2.15) 
and (2.16) hold true for any $r, q \in \mathbb{N}$. Comparing these formulae with Definition 2.3 we find that $\varphi_{n}\left(D^{k} p_{r} p_{q}\right)=D_{n}^{k} \varphi_{n}\left(p_{r} p_{q}\right), r, q \in \mathbb{N}$. Commutativity of the diagram (2.17b) thus follows from the fact that $D^{k}$ and $D_{n}^{k}$ are differential operators of order two, and that $D^{k} p_{0}=0$.

There remains only to prove uniqueness. Suppose that $D, D^{\prime} \in \mathscr{D}\left(\Lambda_{\mathbf{F}}\right)$ are such that $\varphi_{n} \circ$ $\left(D-D^{\prime}\right)=0$ for all $n \in \mathbb{N}$. For any non-zero $p \in \Lambda_{\mathbf{F}}$ there exists $n \in \mathbb{N}$ such that $p \in \Lambda_{\mathbf{F}^{(n)}}$ and $\varphi_{n}(p) \neq 0$. Hence, $D=D^{\prime}$ and the statement follows.

From Lemma 2.4 we can immediately infer the following:

Proposition 2.5. Let

$$
\mathcal{L}=\sum_{k=0}^{\infty} a_{k} D^{k}+\sum_{\ell=0}^{\infty} b_{\ell} E^{\ell}
$$

for some coefficients $a_{k}, b_{\ell} \in \mathbf{F}$ such that only finitely many of them are non-zero. Moreover, let $\mathcal{L}_{n}$ stand for the operator defined in (2.11). Then, $\mathcal{L}$ is a differential operator on $\Lambda_{\mathbf{F}}$. Moreover, it is the unique operator on $\Lambda_{\mathbf{F}}$ such that the diagram

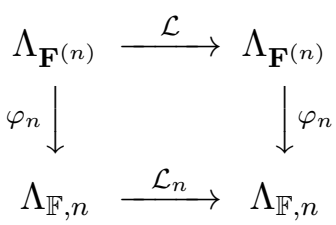

is commutative for all $n \in \mathbb{N}$.

\section{Generalised hypergeometric series}

In this section we define and study a natural analogue of hypergeometric series in the context of symmetric functions, given as formal series in Jack's symmetric functions. When restricted to a finite number of variables, these formal series coincide with (generalised) hypergeometric series studied, in particular, by Korányi [22], Yan [48], Kaneko [18], and Macdonald [28].

We shall first introduce an analogue of Macdonald's hypergeometric series in two sets of variables. For that, we require the graded algebra

$$
\Lambda_{\mathbf{F}} \otimes \Lambda_{\mathbf{F}}=\bigoplus_{k \geq 0}\left(\Lambda_{\mathbf{F}} \otimes \Lambda_{\mathbf{F}}\right)^{k}
$$

where

$$
\left(\Lambda_{\mathbf{F}} \otimes \Lambda_{\mathbf{F}}\right)^{k} \equiv\left\{p_{1} \otimes p_{2}: p_{i} \in \Lambda_{\mathbb{F}}^{k_{i}} \text { with } k_{1}+k_{2}=k\right\} ;
$$

c.f., (2.6). We consider the ideal

$$
U=\bigoplus_{k \geq 1}\left(\Lambda_{\mathbf{F}} \otimes \Lambda_{\mathbf{F}}\right)^{k} \subset \Lambda_{\mathbf{F}} \otimes \Lambda_{\mathbf{F}}
$$

and equip $\Lambda_{\mathbf{F}} \otimes \Lambda_{\mathbf{F}}$ with the structure of a topological ring by requiring that the sequence of ideals $U^{n}, n \in \mathbb{N}_{0}$, form a base of neighbourhoods of $0 \in \Lambda_{\mathbf{F}} \otimes \Lambda_{\mathbf{F}}$. The corresponding completion, hereafter denoted by $\Lambda_{\mathbf{F}} \hat{\otimes} \Lambda_{\mathbf{F}}$, can be identified with the algebra of formal power series

$$
\hat{p}=\sum_{\lambda, \mu} a_{\lambda \mu} p_{\mu} \otimes p_{\lambda}, \quad a_{\mu \lambda} \in \mathbf{F} .
$$

We are now ready to give the precise definition of the hypergeometric series in question. 
Definition 3.1. Fix $p, q \in \mathbb{N}_{0}$ and let $\left(a_{1}, \ldots, a_{p}\right) \in \mathbf{F}^{p}$ and $\left(b_{1}, \ldots, b_{q}\right) \in \mathbf{F}^{q}$ be such that $(i-1) / \alpha-b_{j} \notin \mathbb{N}_{0}$ for all $i \in \mathbb{N}_{0}$. We then define $\mathscr{F}_{q}\left(a_{1}, \ldots, a_{p} ; b_{1}, \ldots, b_{q} ; \alpha, p_{0}\right) \in \Lambda_{\mathbf{F}} \hat{\otimes} \Lambda_{\mathbf{F}}$ by

$$
p^{\mathscr{F}_{q}}\left(a_{1}, \ldots, a_{p} ; b_{1}, \ldots, b_{q} ; \alpha, p_{0}\right)=\sum_{\lambda} \frac{1}{h_{\lambda}} \frac{\left[a_{1}\right]_{\lambda} \cdots\left[a_{p}\right]_{\lambda}}{\left[b_{1}\right]_{\lambda} \cdots\left[b_{q}\right]_{\lambda}} \frac{P_{\lambda} \otimes P_{\lambda}}{\epsilon_{p_{0}}\left(P_{\lambda}\right)},
$$

where $h_{\lambda}$ and $[u]_{\lambda}$ are given by (2.1) and (2.3), respectively.

As in the finite variable case, ${ }_{2} \mathscr{F}_{1}$ satisfies a simple differential equation of second order. In order to make this remark precise, we first note that we can equip also $\Lambda_{\mathbf{F}}$ with the structure of a topological ring by starting from the ideal $U=\oplus_{k \geq 1} \Lambda_{\mathbf{F}}^{k}$. Then, any two continuous differential operators $D_{1}$ and $D_{2}$ on $\Lambda_{\mathbf{F}}$ yield a continuous differential operator $D_{1} \hat{\otimes} D_{2}$ on $\Lambda_{\mathbf{F}} \hat{\otimes} \Lambda_{\mathbf{F}}$ by

$$
D_{1} \hat{\otimes} D_{2}\left(\sum_{\lambda_{1}, \lambda_{2}} a_{\lambda_{1}, \lambda_{2}} p_{\lambda_{1}} \otimes p_{\lambda_{2}}\right) \equiv \sum_{\lambda_{1}, \lambda_{2}} a_{\lambda_{1}, \lambda_{2}}\left(D_{1} p_{\lambda_{1}}\right) \otimes\left(D_{2} p_{\lambda_{2}}\right) .
$$

It is important to note that differential operators that we consider $-E^{\ell}$ and $D^{k}$ for $\ell, k \in \mathbb{N}_{0}-$ are all continuous. For a simple way to see this fact see Lemma A.2 in Appendix A. With this fact in mind, we proceed to state and prove the following:

Proposition 3.2. Let $a, b, c \in \mathbf{F}$ be such that $(i-1) / \alpha-c \notin \mathbb{N}_{0}$ for all $i \in \mathbb{N}_{0}$. Then, ${ }_{2} \mathscr{F}_{1}\left(a, b ; c ; \alpha, p_{0}\right)$ is the unique solution of the differential equation

$$
\begin{aligned}
& \left(D^{1} \hat{\otimes} 1\right) F+\left(c-\frac{p_{0}-1}{\alpha}\right)\left(E^{0} \hat{\otimes} 1\right) F-\left(1 \hat{\otimes} D^{3}\right) F \\
& -\left(a+b+1-\frac{2\left(p_{0}-1\right)}{\alpha}\right)\left(1 \hat{\otimes} E^{2}\right) F=a b\left(1 \hat{\otimes} p_{1}\right) F
\end{aligned}
$$

that is of the form

$$
F=\sum_{\lambda} A_{\lambda} \frac{P_{\lambda} \otimes P_{\lambda}}{h_{\lambda} \epsilon_{p_{0}}\left(P_{\lambda}\right)}, \quad A_{\lambda} \in \mathbf{F}, \quad A_{0}=1 .
$$

Proof. The proof follows closely that of Proposition A.1 in Baker and Forrester [2]. Firstly, we observe that setting $k=2$ in (B.1d) yields

$$
D^{3}=\frac{1}{2}\left[D^{2}, E^{2}\right]+\left(\frac{p_{0}-1}{\alpha}-1\right) E^{2} .
$$

If we now take (3.2) as an ansatz for the solution $F$, then a straightforward, albeit somewhat lengthy, computation using Lemma B.4 shows that the differential equation (3.1) is satisfied if and only if the coefficients $A_{\lambda}$ solve the recurrence relation

$$
\left(c+\lambda_{i}-\frac{(i-1)}{\alpha}\right) A_{\lambda^{(i)}}=\left(a+\lambda_{i}-\frac{(i-1)}{\alpha}\right)\left(b+\lambda_{i}-\frac{(i-1)}{\alpha}\right) A_{\lambda} .
$$

Since we have fixed $A_{0}=1$ and assumed that $(i-1) / \alpha-c \notin \mathbb{N}_{0}$, it is clear that this recurrence relation has a unique solution. Moreover, it follows immediately from the relation

$$
[x]_{\lambda^{(i)}}=[x]_{\lambda}\left(x+\lambda_{i}-\frac{i-1}{\alpha}\right)
$$

that this solution is given by

$$
A_{\lambda}=\frac{[a]_{\lambda}[b]_{\lambda}}{[c]_{\lambda}},
$$

which clearly implies that the series $F$ is equal to ${ }_{2} \mathscr{F}_{1}\left(a, b ; c ; \alpha, p_{0}\right)$. 
The hypergeometric series ${ }_{1} \mathscr{F}_{1},{ }_{0} \mathscr{F}_{1}$ and $\mathscr{F}_{0}$ can be shown to satisfy analogous differential equations. Since we shall make use of this fact in later parts of the paper, we proceed to deduce these differential equations by exploiting suitable limit transitions from ${ }_{2} \mathscr{F}_{1}$. To consider such limits, requires a topology of term-wise convergence of formal power series. For reasons that will become evident below, we shall work with symmetric functions over the real numbers, i.e., with $\Lambda_{\mathbb{R}} \hat{\otimes} \Lambda_{\mathbb{R}}$. Consequently, whenever they occur, we assume that $\alpha, p_{0} \in \mathbb{R}_{+}$. The restriction to positive numbers is made in order to avoid potential singularities of Jack's symmetric functions and $\mathscr{F}_{q}$. However, it is important to note that, since both Jack's symmetric functions as well as all coefficients in ${ }_{p} \mathscr{F}_{q}$ are rational functions of $\alpha$ and $p_{0}$, and the differential operators that are involved are all of finite degree, the differential equations we deduce will hold true also in $\Lambda_{\mathbf{F}} \hat{\otimes} \Lambda_{\mathbf{F}}$.

In order to simplify the exposition somewhat, we shall write $\bar{\lambda}$ to indicate that $\bar{\lambda}=\left(\lambda^{(1)}, \lambda^{(2)}\right)$ for some partitions $\lambda^{(1)}$ and $\lambda^{(2)}$. It will also be convenient to use the corresponding shorthand notation $p_{\bar{\lambda}}=p_{\lambda^{(1)}} \otimes p_{\lambda^{(2)}}$. To each such 'double-partition' $\bar{\lambda}$ we associate a function $C_{\bar{\lambda}}: \Lambda_{\mathbb{R}} \hat{\otimes} \Lambda_{\mathbb{R}} \rightarrow \mathbb{R}$ by the expansion

$$
f=\sum_{\bar{\lambda}} C_{\bar{\lambda}}(f) p_{\bar{\lambda}}, \quad f \in \Lambda_{\mathbb{R}} \hat{\otimes} \Lambda_{\mathbb{R}}
$$

We note that any such function $C_{\bar{\lambda}}$ defines a semi-norm $|\cdot|_{\bar{\lambda}}$ on $\Lambda_{\mathbb{R}} \hat{\otimes} \Lambda_{\mathbb{R}}$ by

$$
|f|_{\bar{\lambda}}=\left|C_{\bar{\lambda}}(f)\right|, \quad f \in \Lambda_{\mathbb{R}} \hat{\otimes} \Lambda_{\mathbb{R}}
$$

where $|\cdot|$ in the right hand side denotes the standard (absolute value) norm on $\mathbb{R}$. The topology of term-wise convergence on $\Lambda_{\mathbb{R}} \hat{\otimes} \Lambda_{\mathbb{R}}$ is now the corresponding natural topology, defined as the weakest topology in which all of these semi-norms, along with addition, are continuous. We note that, equipped with this topology, $\Lambda_{\mathbb{R}} \hat{\otimes} \Lambda_{\mathbb{R}}$ becomes a complete and metrisable locally convex vector space - a so-called Fréchet space. It is important to note that this topology of term-wise convergence does not depend on our specific choice of basis - in the discussion above $p_{\bar{\lambda}}$ with $\bar{\lambda}$ running through all pairs of partitions $\left(\lambda^{(1)}, \lambda^{(2)}\right)$. These latter facts are all easy to infer from the general theory of locally convex vector spaces; see, e.g., Sections V.1-2 in Reed and Simon [39].

We proceed to briefly consider the relation to the $\hat{U}$-adic topology introduced at the beginning of this section. In particular, we observe that, for a sequence $\left\{p_{n}\right\}$ of elements $p_{n} \in \Lambda_{\mathbb{R}} \hat{\otimes} \Lambda_{\mathbb{R}}$, convergence in the $\hat{U}$-adic topology implies term-wise convergence. Moreover, we have the following lemma:

Lemma 3.3. If a differential operator $D$ on $\Lambda_{\mathbb{R}} \hat{\otimes} \Lambda_{\mathbb{R}}$ is continuous in the $\hat{U}$-adic topology, then it is continuous in the topology of term-wise convergence.

Proof. Let $\left\{q_{n}\right\}$ be a sequence of elements $q_{n} \in \Lambda_{\mathbb{R}} \hat{\otimes} \Lambda_{\mathbb{R}}$ such that $q_{n} \rightarrow 0$ term-wise. Fix a 'double'-partition $\bar{\mu}$. By assumption, $D$ is continuous in the $\hat{U}$-adic topology. It follows that there exists $m \in \mathbb{N}_{0}$ such that

$$
D \hat{U}_{m} \subset \hat{U}_{|\bar{\mu}|+1}
$$

We can thus deduce that

$$
\left|D q_{n}\right|_{\bar{\mu}}=\left|\sum_{\bar{\lambda}} C_{q_{n}}(\bar{\lambda}) D p_{\bar{\lambda}}\right|_{\bar{\mu}}=\left|\sum_{|\bar{\lambda}|<m} C_{q_{n}}(\bar{\lambda}) \sum_{\bar{\lambda}^{\prime}} C_{D p_{\bar{\lambda}}}\left(\bar{\lambda}^{\prime}\right) p_{\bar{\lambda}^{\prime}}\right|_{\bar{\mu}} \leq \sum_{|\bar{\lambda}|<m}\left|C_{q_{n}}(\bar{\lambda})\right|\left|C_{D p_{\bar{\lambda}}}(\bar{\mu})\right| .
$$

Hence, the fact that the latter sum is finite implies that $\left|D q_{n}\right|_{\bar{\mu}} \rightarrow 0$. 
We continue by considering limit transitions from the hypergeometric series ${ }_{2} \mathscr{F}_{1}$. For $\gamma \in \mathbb{R}$, let $\sigma_{\gamma}: \Lambda_{\mathbb{R}} \rightarrow \Lambda_{\mathbb{R}}$ be the automorphism given by

$$
\sigma_{\gamma}\left(p_{r}\right)=\gamma^{r} p_{r}, \quad r \in \mathbb{N} .
$$

Since $1 \otimes \sigma_{\gamma}$ is degree preserving, it is continuous, and extends uniquely to a homomorphism $1 \hat{\otimes} \sigma_{\gamma}$ on $\Lambda_{\mathbb{R}} \hat{\otimes} \Lambda_{\mathbb{R}}$. In particular, we have that

$$
\left(1 \hat{\otimes} \sigma_{1 / b}\right)_{2} \mathscr{F}_{1}\left(a, b ; c ; \alpha ; p_{0}\right)=\sum_{\lambda} \frac{[a]_{\lambda}[b]_{\lambda}}{b^{|\lambda|}[c]_{\lambda}} \frac{P_{\lambda} \otimes P_{\lambda}}{\epsilon_{p_{0}}\left(P_{\lambda}\right) h_{\lambda}} .
$$

In the sense of term-wise convergence, this implies the limit

$$
\lim _{b \rightarrow \infty}\left(1 \hat{\otimes} \sigma_{1 / b}\right)_{2} \mathscr{F}_{1}\left(a, b ; c ; \alpha, p_{0}\right)={ }_{1} \mathscr{F}_{1}\left(a ; c ; \alpha, p_{0}\right) .
$$

Consider now the differential equation (3.1) for $F={ }_{2} \mathscr{F}_{1}$, and apply the homomorphism $1 \hat{\otimes} \sigma_{1 / b}$. For any homogeneous differential operator $D$ of finite degree $\operatorname{deg}(D)$, we have that

$$
\sigma_{\gamma} \circ D=\gamma^{\operatorname{deg}(D)} D \circ \sigma_{\gamma}
$$

It follows from Lemma A.2 and Lemma 3.3 that such a differential operator $D$ is continuous with respect to the topology of term-wise convergence, and thereby that it commutes with the limit in question. Using this fact, a direct computation yields the differential equation satisfied by ${ }_{1} \mathscr{F}_{1}$. After computing similar limits in the parameters $a$ and $c$, we arrive at the following proposition:

Proposition 3.4. Let $a, c \in \mathbf{F}$ be such that $(i-1) / \alpha-c \notin \mathbb{N}_{0}$ for all $i \in \mathbb{N}_{0}$. Then, ${ }_{1} \mathscr{F}_{1}\left(a, c ; \alpha, p_{0}\right)$ is a solution of

$$
\left(D^{1} \hat{\otimes} 1\right) F+\left(c-\frac{p_{0}-1}{\alpha}\right)\left(E^{0} \hat{\otimes} 1\right) F-\left(1 \hat{\otimes} E^{2}\right) F=a\left(1 \hat{\otimes} p_{1}\right) F,
$$

${ }_{0} \mathscr{F}_{1}\left(c ; \alpha, p_{0}\right)$ is a solution of

$$
\left(D^{1} \hat{\otimes} 1\right) F+\left(c-\frac{p_{0}-1}{\alpha}\right)\left(E^{0} \hat{\otimes} 1\right) F=\left(1 \hat{\otimes} p_{1}\right) F,
$$

and $\mathscr{F}_{0}\left(\alpha, p_{0}\right)$ is a solution of

$$
\left(E^{0} \hat{\otimes} 1\right) F=\left(1 \hat{\otimes} p_{1}\right) F \text {. }
$$

We conclude this section by briefly considering the hypergeometric series

$$
{ }_{p} F_{q}\left(a_{1}, \ldots, a_{p} ; b_{1}, \ldots, b_{q} ; \alpha, p_{0}\right)=\sum_{\lambda} \frac{1}{h_{\lambda}} \frac{\left[a_{1}\right]_{\lambda} \cdots\left[a_{p}\right]_{\lambda}}{\left[b_{1}\right]_{\lambda} \cdots\left[b_{q}\right]_{\lambda}} P_{\lambda},
$$

which can be obtained by applying the homomorphism $1 \otimes \epsilon_{p_{0}}$ to each term in ${ }_{p} \mathscr{F}_{q}$. In this equation, it is assumed that the indeterminates $\left(b_{1}, \ldots, b_{q}\right)$ comply with the conditions stated in Definition 3.1. The next Proposition generalises a result of Yan [48] and Kaneko [18] on the solution of a multivariable generalisation of Euler's hypergeometric equation. The proof is omitted since it closely parallels that of Proposition 3.2.

Proposition 3.5. Let $a, b, c \in \mathbf{F}$ be such that $(i-1) / \alpha-c \notin \mathbb{N}_{0}$ for all $i \in \mathbb{N}_{0}$. Then, ${ }_{2} F_{1}\left(a, b ; c ; \alpha, p_{0}\right)$ is the unique solution of the differential equation

$$
D^{1} F-D^{2} F+\left(c-\frac{p_{0}-1}{\alpha}\right) E^{0} F-\left(a+b+1-\frac{p_{0}-1}{\alpha}\right) E^{1} F=a b p_{0} F
$$

that is of the form

$$
F=\sum_{\lambda} \frac{A_{\lambda}}{h_{\lambda}} P_{\lambda}, \quad A_{\lambda} \in \mathbf{F}, \quad A_{0}=1 .
$$




\section{Hermite symmetric functions}

In this section we introduce and study Hermite symmetric functions as eigenfunctions of the differential operator

$$
\mathcal{L}^{H}=D^{0}-2 \nu^{2} E^{1}
$$

with the parameter $\nu \in \mathbf{F}$. As in the finite variable case, one can essentially remove the dependence on the parameter $\nu$. More precisely, since $D^{0}$ and $E^{1}$ are of degree -2 and 0 , respectively, we have that

$$
\sigma_{1 / \nu} \circ\left(D^{0}-2 \nu^{2} E^{1}\right)=\nu^{2}\left(D^{0}-2 E^{1}\right) \circ \sigma_{1 / \nu}
$$

c.f., (3.4). Using this fact, we can reduce most of the statements below to that for a fixed value of $\nu$. However, $\nu$ will play an important role in our discussion of a particular duality of the Hermite symmetric functions. For the moment we therefore refrain from specifying a fixed value for $\nu$.

It is readily inferred from Lemma B.4 that

$$
\mathcal{L}^{H} P_{\lambda}=-2 \nu^{2}|\lambda| P_{\lambda}+\sum_{\mu \subset \lambda} c_{\lambda_{\mu}} P_{\mu}
$$

for some coefficients $c_{\lambda \mu} \in \mathbf{F}$. By Theorem B.6, it is thus clear that we can make the following definition:

Definition 4.1. Let $\lambda$ be a partition. We then define the Hermite symmetric function $H_{\lambda}\left(\alpha, p_{0}, \nu^{2}\right)$ as the unique symmetric function such that

1. $H_{\lambda}=P_{\lambda}+\sum_{\mu \subset \lambda} u_{\lambda \mu} P_{\mu}$ for some $u_{\lambda \mu} \in \mathbf{F}$,

2. $\mathcal{L}^{H} H_{\lambda}=-2 \nu^{2}|\lambda| H_{\lambda}$.

Remark 4.2. The generalised Hermite polynomials are recovered by setting $p_{0}=n$ and restricting to $n$ indeterminates $x=\left(x_{1}, \ldots, x_{n}\right)$. Indeed, using Proposition 2.5 it is readily verified that the resulting symmetric polynomials satisfy definitions given by Lassalle [26] and Macdonald [28].

Before proceeding to further investigate the properties of the Hermite symmetric functions, we detail a constructive definition in terms of the Jack symmetric functions; c.f., (3.21) in Baker and Forrester [2] for the corresponding result in the finite variable case. This requires the following notation: given a differential operator $D$ on $\Lambda_{\mathbf{F}}$ and $L \in \mathbb{N}$, we let

$$
\exp _{L}(D)=1+\sum_{k=1}^{L} \frac{1}{k !}(D)^{k} .
$$

Clearly, $\exp _{L}(D)$ is a differential operator on $\Lambda_{\mathbf{F}}$. Furthermore, if $D$ has finite degree (see the paragraph preceding Lemma A.2), then so has $\exp _{L}(D)$, which, by Lemma A.2, implies continuity. We stress the importance of truncating the series in the right-hand side of (4.3) at some positive integer $L$. Indeed, if this is not done, then we do not obtain a differential operator on $\Lambda_{\mathbf{F}}$, c.f. the paragraph containing (A.3).

Proposition 4.3. For any $L \geq\lfloor|\lambda| / 2\rfloor$, we have that

$$
H_{\lambda}=\exp _{L}\left(-\frac{1}{4 \nu^{2}} D^{0}\right)\left(P_{\lambda}\right)
$$


Proof. For simplicity of exposition, we let $\Delta=-\frac{1}{4 \nu^{2}} D^{0}$. It follows immediately from (B.6f) in Lemma B.4 that $\exp _{L}(\Delta)\left(P_{\lambda}\right)$ satisfies property (1) in Definition 4.1. Since $E^{1} P_{\lambda}=|\lambda| P_{\lambda}$ and $\left[E^{1}, \Delta\right]=-2 \Delta$, we have

$$
E^{1}\left(\Delta^{k}\left(P_{\lambda}\right)\right)=(|\lambda|-2 k) \Delta^{k}\left(P_{\lambda}\right) .
$$

Consequently,

$$
\begin{aligned}
\mathcal{L}^{H}\left(\exp _{L}(\Delta)\left(P_{\lambda}\right)\right)= & -2 \nu^{2}\left(E^{1}+2 \Delta\right)\left(P_{\lambda}+\Delta\left(P_{\lambda}\right)+\frac{1}{2 !} \Delta^{2}\left(P_{\lambda}\right)+\cdots+\frac{1}{L !} \Delta^{L}\left(P_{\lambda}\right)\right) \\
= & -2 \nu^{2}\left(|\lambda| P_{\lambda}+(|\lambda|-2) \Delta\left(P_{\lambda}\right)+\frac{(|\lambda|-4)}{2 !} \Delta^{2}\left(P_{\lambda}\right)+\cdots\right. \\
& \left.+\frac{|\lambda|-2 L}{L !} \Delta^{L}\left(P_{\lambda}\right)+2 \Delta\left(P_{\lambda}\right)+2 \Delta^{2}\left(P_{\lambda}\right)+\cdots+\frac{2}{(L-1) !} \Delta^{L}\left(P_{\lambda}\right)\right) \\
= & -2 \nu^{2}|\lambda| \exp _{L}(\Delta)\left(P_{\lambda}\right),
\end{aligned}
$$

i.e., also property $(2)$ is satisfied by $\exp _{L}(\Delta)\left(P_{\lambda}\right)$.

\subsection{A duality relation}

We proceed to establish a particular duality relation for the Hermite symmetric functions that is not present at the level of the corresponding symmetric polynomials. To this end, we recall the standard automorphism $\omega_{\gamma}, \gamma \in \mathbb{F}$, of $\Lambda_{\mathbb{F}}$, given by

$$
\omega_{\gamma}\left(p_{r}\right)=(-1)^{r-1} \gamma p_{r}, \quad r \in \mathbb{N} .
$$

It is well known that, for a given value of the parameter $\alpha$, Jack's symmetric functions corresponding to the inverse parameter value $1 / \alpha$ can be obtained by the following duality relation:

$$
\omega_{\alpha}\left(P_{\lambda}(\alpha)\right)=Q_{\lambda^{\prime}}(1 / \alpha)
$$

where

$$
Q_{\lambda}=b_{\lambda} P_{\lambda}, \quad b_{\lambda}=\prod_{(i, j) \in \lambda} \frac{l_{\lambda}(i, j)+1+\alpha a_{\lambda}(i, j)}{l_{\lambda}(i, j)+\alpha+\alpha a_{\lambda}(i, j)}
$$

see, e.g., Section VI.10 in Macdonald [29]. This duality relation can be inferred from the identity

$$
-\alpha\left(\omega_{\alpha} \circ D(\alpha)\right)=D(1 / \alpha), \quad D=D^{2}-\frac{2}{\alpha}\left(p_{0}-1\right) E^{1},
$$

and the fact that Jack's symmetric functions can be defined as the unique eigenfunctions of $D$ that are of the form (2.8); c.f., (2.7); and see Lemma 4.4 below and note that $D$ is independent of $p_{0}$.

In order to deduce an analogous duality relation for the Hermite symmetric functions, we must consider also the parameter $p_{0}$. The reason being that these symmetric functions have no eigenoperators that are independent of $p_{0}$. We therefore extend the automorphism $\omega_{\gamma}$ to $\Lambda_{\mathbf{F}}$ by setting

$$
\omega_{\gamma}\left(p_{0}\right)=-\gamma p_{0},
$$

or, equivalently, by replacing $\mathbb{N}$ by $\mathbb{N}_{0}$ in (4.5). With this extension in force, it is straightforward to determine the effect of $\omega_{\alpha}$ on the CMS operators $E^{\ell}$ and $D^{k} \equiv D^{k}\left(\alpha, p_{0}\right)$. 
Lemma 4.4. We have that

$$
\omega_{\alpha} \circ E^{\ell}=(-1)^{\ell-1} E^{\ell} \circ \omega_{\alpha}
$$

and

$$
(-1)^{k-1} \alpha\left(\omega_{\alpha} \circ D^{k}\left(\alpha, p_{0}\right)\right)=D^{k}\left(1 / \alpha,-\alpha p_{0}\right) \circ \omega_{\alpha}-(\alpha+1) k\left(E^{k-1} \circ \omega_{\alpha}\right) .
$$

Proof. It follows immediately from (4.5) that

$$
\begin{aligned}
& \omega_{\alpha} \circ p_{r}=(-1)^{r-1} \alpha\left(p_{r} \circ \omega_{\alpha}\right), \quad r \in \mathbb{N}_{0}, \\
& \omega_{\alpha} \circ \partial\left(p_{r}\right)=\frac{(-1)^{r-1}}{\alpha}\left(\partial\left(p_{r}\right) \circ \omega_{\alpha}\right), \quad r \in \mathbb{N} .
\end{aligned}
$$

Using these relations, a direct computation yields the statement for $E^{\ell}$. We continue by observing that

$$
\begin{aligned}
D^{k}+\frac{k}{\alpha} E^{k-1}= & \sum_{r, q=1}^{\infty} r q p_{r+q+k-2} \partial\left(p_{r}\right) \partial\left(p_{q}\right) \\
& +\sum_{r=2}^{\infty} r(r-1)\left(1-\frac{1}{\alpha}\right) p_{r+k-2} \partial\left(p_{r}\right)+\frac{1}{\alpha} \sum_{r=1}^{\infty} r \sum_{m=0}^{r+k-2} p_{r+k-2-m} p_{m} \partial\left(p_{r}\right) .
\end{aligned}
$$

Using again $(4.8 \mathrm{a})$, it is readily seen that

$$
\omega_{\alpha} \circ\left(D^{k}\left(\alpha, p_{0}\right)+\frac{k}{\alpha} E^{k-1}\right)=\frac{(-1)^{k-1}}{\alpha}\left(D^{k}\left(1 / \alpha,-\alpha p_{0}\right)-k \alpha E^{k-1}\right) \circ \omega_{\alpha}
$$

which clearly implies the statement for $D^{k}$.

There are now (at least) two different methods by which we can establish a duality relation for the Hermite symmetric functions. Firstly, we can follow the method sketched above for Jack's symmetric functions; and, secondly, we can make use of the representation (4.4). Here, we shall employ the latter method, since it yields a somewhat shorter proof.

Proposition 4.5. We have the duality relation

$$
\omega_{\alpha}\left(H_{\lambda}\left(\alpha, p_{0}, \nu^{2}\right)\right)=b_{\lambda^{\prime}}(1 / \alpha) H_{\lambda^{\prime}}\left(1 / \alpha,-\alpha p_{0},-\alpha \nu^{2}\right) .
$$

Proof. Starting from (4.4), we infer from Lemma 4.4 that

$$
\omega_{\alpha}\left(H_{\lambda}\left(\alpha, p_{0}, \nu^{2}\right)\right)=\exp _{L}\left(\frac{1}{4 \alpha \nu^{2}} D^{0}\left(1 / \alpha,-\alpha p_{0}\right)\right)\left(Q_{\lambda^{\prime}}(1 / \alpha)\right) .
$$

The statement is now a direct consequence of (4.7).

We stress that the duality relation (4.5) has no direct analogue in the finite variable case. Indeed, the 'restriction' homomorphism $\varphi_{n}$, which maps $H_{\lambda}$ to $H_{\lambda}\left(x_{1}, \ldots, x_{n}\right)$, fixes $p_{0}=n$, whereas $\omega_{\alpha}$ maps $p_{0}$ to $-\alpha p_{0}$, and thus can not be restricted to $\Lambda_{\mathbb{F}, n}=\varphi_{n}\left(\Lambda_{\left.\mathbf{F}^{(n)}\right)}\right)$. However, this duality relation does have a natural analogue for the super Hermite polynomials, introduced in Section 6.3.

In the remainder of this section the parameter $\nu$ will not play any particular role. From hereon, we shall therefore assume that $\nu=1$. If needed, then this parameter can be reintroduced by applying the automorphism $\sigma_{\nu}$; c.f. the paragraph containing (4.2). 


\subsection{A generating function}

We proceed to establish a generating function for the Hermite symmetric functions. As a first example of its usefulness, we shall then use this generating function to construct higher-order eigenoperators for the Hermite symmetric functions. These results will be obtained as rather direct generalisations of corresponding results due to Baker and Forrester [2] on the generalised Hermite polynomials - in turn based on an unpublished manuscript by Lassalle.

Proposition 4.6. We have that

$$
\sum_{\lambda} \frac{1}{h_{\lambda} \epsilon_{p_{0}}\left(P_{\lambda}\right)} H_{\lambda} \otimes P_{\lambda}={ }_{0} \mathscr{F}_{0} e^{-\frac{1}{4}\left(1 \otimes p_{2}\right)}
$$

with

$$
e^{-\frac{1}{4}\left(1 \otimes p_{2}\right)}:=\sum_{n=0}^{\infty} \frac{1 \otimes\left(-p_{2} / 4\right)^{n}}{n !} .
$$

Proof. Let $x=\left(x_{1}, \ldots, x_{n}\right)$ and $y=\left(y_{1}, \ldots, y_{n}\right)$ be two sequences of indeterminates. By writing $\varphi_{n, x}$ and $\varphi_{n, y}$ we indicate that the homomorphism map $\Lambda_{\mathbf{F}^{(m)}}$ onto the algebra of symmetric polynomials in the indeterminates $x$ and $y$, respectively. We have that

$$
\left(\varphi_{n, x} \hat{\otimes} \varphi_{n, y}\right)_{0} \mathscr{F}_{0} e^{-\frac{1}{4}\left(1 \otimes p_{2}\right)}={ }_{0} \mathscr{F}_{0}(x, y) e^{-\frac{1}{4}\left(1 \otimes p_{2}(y)\right)},
$$

where

$$
{ }_{0} \mathscr{F}_{0}(x ; y)=\sum_{\lambda} \frac{1}{h_{\lambda} P_{\lambda}\left(1^{n}\right)} P_{\lambda}(x) \otimes P_{\lambda}(y)
$$

and $e^{-\frac{1}{4}\left(1 \otimes p_{2}(y)\right)}$ is defined in the obvious way. As shown by Baker and Forrester [2] (see their Proposition 3.1),

$$
{ }_{0} \mathscr{F}_{0}(x ; y) e^{-\frac{1}{4}\left(1 \otimes p_{2}(y)\right)}=\sum_{\lambda} \frac{1}{h_{\lambda} P_{\lambda}\left(1^{n}\right)} H_{\lambda}(x) \otimes P_{\lambda}(y),
$$

where the sum is over all partitions $\lambda$ such that $\ell(\lambda) \leq n$. We note that Baker and Forrester use the normalisation $C_{\lambda}(x)=|\lambda| ! P_{\lambda}(x) / h_{\lambda}$ for the Jack polynomials, and that their generalised Hermite polynomials are equal to $2^{|\lambda|} H_{\lambda}(x) / P_{\lambda}\left(1^{n}\right)$. As a consequence, the generating function expansion (4.12) differs slightly from that stated by Baker and Forrester.

By a direct expansion of the right hand side of (4.10) in terms of Jack symmetric functions we obtain

$$
{ }_{0} \mathscr{F}_{0} e^{-\frac{1}{4}\left(1 \otimes p_{2}\right)}=\sum_{\lambda} \frac{1}{h_{\lambda} \epsilon_{p_{0}}\left(P_{\lambda}\right)} U_{\lambda} \otimes P_{\lambda}
$$

for some $U_{\lambda} \in \Lambda_{\mathbf{F}}$. Using (B.1e) for $k=2$ and $\ell=0$, (B.6a) and Proposition B.3, it is readily verified that these symmetric functions are of the form

$$
U_{\lambda}=\sum_{\mu \subset \lambda} v_{\lambda \mu} P_{\lambda}, \quad v_{\lambda \mu} \in \mathbf{F}
$$

If we compare the two expansions (4.12) and (4.13), then we find that

$$
\varphi_{n, x}\left(U_{\lambda}\right)=H_{\lambda}(x) \equiv \varphi_{n, x}\left(H_{\lambda}\right), \quad \forall n \geq \ell(\lambda) .
$$

Since both $U_{\lambda}$ and $H_{\lambda}$ depend rationally on $p_{0}$, i.e., when expanded in, e.g., Jack's symmetric functions, the coefficients are rational functions of $p_{0}$, it follows that $U_{\lambda}=H_{\lambda}$. 
Proposition 4.6 can also be established from first principles by essentially the same method used by Baker and Forrester [2] to prove (4.12).

The generating function (4.10) is an effective tool for establishing a number of basic properties of the Hermite symmetric functions. For example, the effect of multiplication by $p_{1}$ and application of the differential operator $E_{0}$. However, since these results can be obtained in complete analogy with the proofs of Corollaries 3.4 and 3.5 in Baker and Forrester [2], we leave it to the interested reader to work out the details. Furthermore, the recurrence relation corresponding to multiplication by $p_{1}$ is the simplest special case of the complete set of recurrence relations we shall obtain in Section 4.4; see Proposition 4.18.

We proceed to use Proposition 4.6 to obtain higher-order eigenoperators for the Hermite symmetric functions. Also this result can be deduced in close analogy with Proposition 3.2 in Baker and Forrester [2]. At this point it might be helpful to recall the discussion of eigenoperators for Jack's symmetric functions in Section 2.3.

Let $D$ be a differential operator of order $k$ on $\Lambda_{\mathbf{F}} \hat{\otimes} \Lambda_{\mathbf{F}}$ (see Appendix A for the definition of order). It follows from the Baker-Campbell-Hausdorff formula that, for any $f \in \Lambda_{\mathbf{F}} \hat{\otimes} \Lambda_{\mathbf{F}}$,

$$
\begin{aligned}
D f e^{-\frac{1}{4}\left(1 \otimes p_{2}\right)}=e^{-\frac{1}{4}\left(1 \otimes p_{2}\right)}( & D+\frac{1}{4}\left[\left(1 \hat{\otimes} p_{2}\right), D\right]+\frac{1}{4^{2} 2 !}\left[\left(1 \hat{\otimes} p_{2}\right),\left[\left(1 \hat{\otimes} p_{2}\right), D\right]\right] \\
& \left.+\cdots+\frac{1}{4^{k} k !}\left[\left(1 \hat{\otimes} p_{2}\right), \ldots,\left[\left(1 \hat{\otimes} p_{2}\right), D\right] \cdots\right]\right) f
\end{aligned}
$$

where $1 \hat{\otimes} p_{2}$ denotes the operator of multiplication by $1 \otimes p_{2}$. From Definition 3.1 (for $p=q=0$ ) we can directly infer that

$$
\left(\mathcal{L}_{f} \hat{\otimes} 1\right)_{0} \mathscr{F}_{0}=\left(1 \hat{\otimes} \mathcal{L}_{f}\right)_{0} \mathscr{F}_{0}, \quad \forall f \in \Lambda_{\mathbb{F}, \alpha},
$$

where $\mathcal{L}_{f}$ denotes the eigenoperator for Jack's symmetric functions given by (2.9). Moreover, since $p_{2}=\left[E^{2}, p_{1}\right]$, we can infer from Proposition B.3 and Lemma B.4 that

$$
\left(D^{0} \hat{\otimes} 1\right)_{0} \mathscr{F}_{0}=\left(1 \hat{\otimes} p_{2}\right)_{0} \mathscr{F}_{0} .
$$

Using these facts, as well as (4.15) for $D=1 \hat{\otimes} \mathcal{L}_{f}$, it is a matter of straightforward computations to verify that the following Proposition holds true:

Proposition 4.7. Let $f \in \Lambda_{\mathbb{F}, \alpha}$, and let $k$ be the degree of $f$. Then, we have that

$$
\left(1 \hat{\otimes} \mathcal{L}_{f}\right)_{0} \mathscr{F}_{0} e^{-\frac{1}{4}\left(1 \otimes p_{2}\right)}=\left(\mathcal{L}_{f}^{H} \hat{\otimes} 1\right)_{0} \mathscr{F}_{0} e^{-\frac{1}{4}\left(1 \otimes p_{2}\right)},
$$

where

$$
\mathcal{L}_{f}^{H}=\mathcal{L}_{f}-\frac{1}{4}\left[D^{0}, \mathcal{L}_{f}\right]+\frac{1}{4^{2} 2 !}\left[D^{0},\left[D^{0}, \mathcal{L}_{f}\right]\right]+\cdots+\frac{(-1)^{k}}{4^{k} k !}\left[D^{0}, \ldots,\left[D^{0}, \mathcal{L}_{f}\right] \cdots\right] .
$$

In particular, the set of differential operators $\mathcal{L}_{f}^{H}, f \in \Lambda_{\mathbb{F}, \alpha}$, contains the CMS operator $\mathcal{L}^{H}$. Indeed, it is readily verified that

$$
\mathcal{L}^{H}=\mathcal{L}_{-2 \pi_{1, \alpha}}^{H}
$$

note (4.1) and (B.1c), and use the fact that $\mathcal{L}_{-2 \pi_{1, \alpha}}=-2 E^{1}$. We also note that if we substitute $\mathcal{L}_{f, n}$ for $\mathcal{L}_{f}$ and $D_{n}^{0}$ for $D^{0}$ in $(4.16)$, then we obtain an eigenoperator $\mathcal{L}_{f, n}^{H}$ for the generalised Hermite polynomials, which satisfies the intertwining relation

$$
\varphi_{n} \circ \mathcal{L}_{f}=\mathcal{L}_{f, n} \circ \varphi_{n}
$$


Now, in any differential operator in $n$ variables, let l.o. denote terms of lower order. Given that the Cherednik operators $\xi_{i}$ satisfy $\xi_{i}=x_{i} \frac{\partial}{\partial x_{i}}+$ l.o. (see for instance [43, Section 4]), it is clear from (2.9) that

$$
\mathcal{L}_{f, n}=f\left(x_{1} \frac{\partial}{\partial x_{1}}, \ldots, x_{n} \frac{\partial}{\partial x_{n}}\right)+\text { l.o.. }
$$

Given that $D_{n}^{0}$ is a differential operator of order two, this implies that the order of $\left[D_{n}^{0}, \mathcal{L}_{f, n}\right]$ is $k+1$, that of $\left[D_{n}^{0},\left[D_{n}^{0}, \mathcal{L}_{f, n}\right]\right]$ is $k+2$, etc. In particular, this means that the order of $\mathcal{L}_{f, n}^{H}$, and therefore also of $\mathcal{L}_{f}^{H}$, is $2 k$. As a consequence of Propositions 4.6 and 4.7, we thus obtain the following corollary:

Corollary 4.8. Let $f$ be as in Proposition 4.7. Then, $\mathcal{L}_{f}^{H}$ is a differential operator on $\Lambda_{\mathbf{F}}$ of order $2 k$. Moreover, it is the unique operator on $\Lambda_{\mathbf{F}}$ such that

$$
\mathcal{L}_{f}^{H} H_{\lambda}=f(\lambda) H_{\lambda}
$$

for all partitions $\lambda$.

Proof. There remains only to prove uniqueness, but this is immediate from the fact that the Hermite symmetric functions span $\Lambda_{\mathbf{F}}$; c.f., Corollary B.7.

Referring again to the fact that the Hermite symmetric functions form a basis for $\Lambda_{\mathbf{F}}$, we can conclude that the eigenoperators $\mathcal{L}_{f}^{H}$ pairwise commute.

Corollary 4.9. We have that

$$
\left[\mathcal{L}_{f}^{H}, \mathcal{L}_{g}^{H}\right]=0
$$

for all $f, g \in \Lambda_{\mathbb{F}, \alpha}$.

We also note that the set of eigenoperators $\mathcal{L}_{f}^{H}, f \in \Lambda_{\mathbb{F}, \alpha}$, separate the Hermite symmetric functions.

Lemma 4.10. For any two partitions $\lambda$ and $\mu$ such that $\lambda \neq \mu$, there exists $f \in \Lambda_{\mathbb{F}, \alpha}$ such that $f(\lambda) \neq f(\mu)$.

Proof. Let

$$
\lambda^{\alpha}=\left(\lambda_{1}, \lambda_{2}-1 / \alpha, \ldots, \lambda_{i}-(i-1) / \alpha, \ldots, \lambda_{\ell(\lambda)}-(\ell(\lambda)-1) / \alpha\right)
$$

and similarly for $\mu^{\alpha}$. For any $f \in \Lambda_{\mathbb{F}, \alpha}$, there exists a unique $p_{f} \in \Lambda_{\mathbb{F}}$ such that $f(\lambda)=p_{f}\left(\lambda^{\alpha}\right)$, and vice versa. If we expand $p_{f}(\lambda)$ in powers of $\alpha$, then we obtain

$$
p_{f}\left(\lambda^{\alpha}\right)=p_{f}(\lambda)+\text { l.d. }
$$

where l.d. stands for terms of lower degree in $\alpha$. Since $\alpha$ is an indeterminate, we can conclude that $f(\lambda)=f(\mu)$ if and only if $p_{f}(\lambda)=p_{f}(\mu)$. The fact that the symmetric functions separate partitions thus implies the statement. 


\subsection{A limit from the Jacobi symmetric functions}

As indicated in the introduction, Sergeev and Veselov [41] introduced and studied Jacobi symmetric functions as eigenfunctions of the differential operator

$$
\mathcal{L}^{J}=D^{2}+2 D^{1}-(p+2 q-1) E^{1}-(2 p+2 q-1) E^{0} .
$$

To make matters precise, let $\lambda$ be a partition. By Theorem B.6, we can then define a corresponding Jacobi symmetric function $\mathcal{J}_{\lambda}\left(\alpha, p_{0}, p, q\right)$ as the unique eigenfunction of the differential operator $\mathcal{L}^{J}$ that is of the form

$$
\mathcal{J}_{\lambda}=P_{\lambda}+\sum_{\mu \subset \lambda} u_{\lambda \mu} P_{\mu}, \quad u_{\lambda \mu} \in \Lambda_{\mathbf{F}(p, q)} .
$$

The associated eigenvalue is given by

$$
e^{J}(\lambda)=\sum_{i} \lambda_{i}\left(\lambda_{i}+\frac{2}{\alpha}\left(p_{0}-i\right)\right)-(p+2 q-1)|\lambda|
$$

We recall that Sergeev and Veselov used the parameter $k=-1 / \alpha$, specified the form of the Jacobi symmetric functions in terms of the symmetric monomials $m_{\lambda}$, and fixed the leading coefficient to $2^{|\lambda|}$. However, it is readily inferred from the triangular expansion (2.8) and the fact that the dominance order is compatible with the order given by inclusion of diagrams (see the discussion succeeding Theorem B.6) that the definition given above is, up to a difference in normalisation, equivalent to that given by Sergeev and Veselov.

We note that the one-variable polynomials

$$
\mathcal{J}_{n}(x)=\varphi_{1}\left(\mathcal{J}_{(n)}\right), \quad n \in \mathbb{N}_{0},
$$

have a somewhat non-standard form. Indeed,

$$
\varphi_{1} \circ \mathcal{L}^{J}=\left(x(x+2) \frac{d^{2}}{d x^{2}}-((p+2 q-1) x+2 p+2 q-1) \frac{d}{d x}\right) \circ \varphi_{1},
$$

and the $\mathcal{J}_{n}(x)$ can be seen to form a sequence of orthogonal polynomials on the interval $[-2,0]$ with respect to the weight function

$$
w(x)=x^{-p-q-1 / 2}(2+x)^{-q-1 / 2}
$$

for appropriate parameter values. In order to obtain a more standard one-variable restriction, we can instead start from the symmetric functions

$$
J_{\lambda}:=\frac{1}{(-2)^{|\lambda|}} \sigma_{-2}\left(\mathcal{J}_{\lambda}\right)
$$

which are (monic) eigenfunctions of $\sigma_{-2} \circ \mathcal{L}^{J} \circ \sigma_{-1 / 2}$. Using (3.4), we find that

$$
\begin{aligned}
\varphi_{1} \circ\left(\sigma_{-2} \circ \mathcal{L}^{J} \circ \sigma_{-1 / 2}\right) & =\varphi_{1}\left(D^{2}-D^{1}-(p+2 q-1) E^{1}+(p+q-1 / 2) E^{0}\right) \\
& =\left(x(x-1) \frac{d^{2}}{d x^{2}}-((p+2 q-1) x-p-q+1 / 2) \frac{d}{d x}\right) \circ \varphi_{1},
\end{aligned}
$$

and it is readily inferred that the polynomials $J_{n}(x):=\varphi_{1}\left(J_{(n)}\right)$ are orthogonal on the interval $[0,1]$ with respect to the weight function

$$
\tilde{w}(x)=x^{-p-q-1 / 2}(x-1)^{-q-1 / 2} .
$$


Moreover, after a suitable reparameterisation and renormalisation, the symmetric polynomials $J_{\lambda}\left(x_{1}, \ldots, x_{n}\right):=\varphi_{n}\left(J_{\lambda}\right)$ coincide with the generalised Jacobi polynomials, as considered by Lassalle [25], Macdonald [28] and also by Baker and Forrester [2].

We shall now use a standard method to obtain the Hermite symmetric functions as a particular limit of the Jacobi symmetric functions. In order to do so, we shall again work with symmetric functions over real numbers, i.e., with $\Lambda_{\mathbb{R}}$, and thus assume that $\alpha, p_{0}, p, q \in \mathbb{R}$. The starting point is the representation (B.9), which in this case yields

$$
\mathcal{J}_{\lambda}=\prod_{\mu \subset \lambda} \frac{\mathcal{L}^{J}-e^{J}(\mu)}{e^{J}(\lambda)-e^{J}(\mu)} P_{\lambda} .
$$

If we replace $\mathcal{L}^{J}$ by $\mathcal{L}^{H}$ and $e^{J}(\lambda)$ by $-2|\lambda|$, then we obtain the corresponding representation for the Hermite symmetric functions $H_{\lambda}$.

We now introduce a homomorphism $t_{\gamma}: \Lambda_{\mathbf{F}} \rightarrow \Lambda_{\mathbf{F}}, \gamma \in \mathbb{F}$, by setting

$$
t_{\gamma}\left(p_{r}\right)=\sum_{m=0}^{r} \gamma^{r-m}\left(\begin{array}{c}
r \\
m
\end{array}\right) p_{m}, \quad r \geq 1 .
$$

For a finite number of indeterminates $x=\left(x_{1}, \ldots, x_{n}\right)$, this simply yields the translation of each $x_{i}$ by $\gamma$. It follows from Lemma B.8 and (3.4), that

$$
\left(\sigma_{(-q)^{-1 / 2}} \circ t_{-1}\right) \circ \mathcal{L}^{J}=\left(D^{2}+q D^{0}-(p+2 q-1) E^{1}-(-q)^{1 / 2} p E^{0}\right) \circ\left(\sigma_{(-q)^{-1 / 2}} \circ t_{-1}\right) .
$$

We note that, by Lemma 2.4, Lemma A.2 and (the obvious analogue for $\Lambda_{\mathbb{R}}$ of) Lemma 3.3, the differential operator $\mathcal{L}^{J}$ is continuous with respect to the topology of term-wise convergence. Combining the observations above with the binomial formula in Proposition B.2, as well as the fact that Jack's symmetric functions $P_{\lambda}$ are homogeneous of degree $|\lambda|$, we readily deduce the following proposition:

Proposition 4.11. Let $\lambda$ be a partition. Then, for generic parameter values, we have that

$$
H_{\lambda}\left(\alpha, p_{0}\right)=\lim _{q \rightarrow \infty}(-q)^{|\lambda| / 2}\left(\sigma_{(-q)^{-1 / 2}} \circ t_{-1}\right)\left(\mathcal{J}_{\lambda}\left(\alpha, p_{0}, p, q\right)\right)
$$

in the sense of term-wise convergence.

Remark 4.12. By generic we mean on a dense set in parameter space with respect to the Zariski topology. The validity of this part of the statement is a direct consequence of the fact that the Jacobi symmetric functions $J_{\lambda}\left(\alpha, p_{0}, p, q\right)$ depend rationally on all parameters.

\subsection{Structure of Pieri formulae and invariant ideals}

Throughout this section we shall assume $p_{0} \in \mathbb{F}$ fixed. The main purpose is to obtain the ideals $I \subset \Lambda_{\mathbb{F}}$ that are invariant under the action of all differential operators $\mathcal{L}_{f}^{H}, f \in \Lambda_{\mathbb{F}, \alpha}$. This is the case if and only if $I$ has a basis consisting of Hermite symmetric functions. The first part of this claim is trivial, while the second part is a consequence of Lemma 4.10. For future reference, we state this fact in the form of a lemma.

Lemma 4.13. Let $I \subset \Lambda_{\mathbb{F}}$ be an ideal such that $\mathcal{L}_{f}^{H} I \subset I$ for all $f \in \Lambda_{\mathbb{F}, \alpha}$. Then, we have that

$$
I=\mathbb{F}\left\langle H_{\lambda}: \lambda \in \operatorname{Par}_{I}\right\rangle
$$

for some set of partitions $\operatorname{Par}_{I}$. 
We proceed to deduce Pieri type recurrence relations for the Hermite symmetric functions. In the Jacobi case, Sergeev and Veselov [41] (see Theorem 4.4) obtained such recurrence relations by generalising corresponding recurrence relations for generalised Jacobi polynomials due to van Diejen [47] (see Theorem 6.4). However, in this generalisation part of the explicit nature of van Diejen's formulae were lost. In fact, for our purposes, we require a more explicit version of Sergeev and Veselov's result, stated below in Theorem 4.15. By applying the limit transition in Proposition 4.11 we shall then obtain the desired recurrence relations for the Hermite symmetric functions.

An important ingredient is the specialisation formula of the Jacobi symmetric functions at $p_{r}=0, r \in \mathbb{N}$, as deduced by Sergeev and Veselov [41, Proposition 4.3] from the analogue formula for the finite-dimensional case (i.e., $p_{0}=n$ ). The latter can be obtained from Corollary 5.2 [36] by specialising to the root system $B C_{n}$.

Proposition 4.14 (Sergeev and Veselov [41]). For any partition $\lambda$, let

$$
\begin{aligned}
& C_{\lambda}^{+}(z ; \alpha)=\prod_{(i, j) \in \lambda}\left(\lambda_{i}+j-\left(\lambda_{j}^{\prime}+i\right) / \alpha+z\right), \\
& C_{\lambda}^{-}(z ; \alpha)=\prod_{(i, j) \in \lambda}\left(\lambda_{i}-j+\left(\lambda_{j}^{\prime}-i\right) / \alpha+z\right), \\
& C_{\lambda}^{0}(z ; \alpha)=\prod_{(i, j) \in \lambda}(j-1-(i-1) / \alpha+z) .
\end{aligned}
$$

Then, we have that

$$
\epsilon_{0}\left(\mathcal{J}_{\lambda}\left(\alpha, p_{0}, p, q\right)\right)=2^{|\lambda|} \frac{C_{\lambda}^{0}\left(p_{0} / \alpha\right) C_{\lambda}^{0}\left(\left(p_{0}-1\right) / \alpha-p-q+1 / 2\right)}{C_{\lambda}^{-}(1 / \alpha) C_{\lambda}^{+}\left(2 p_{0} / \alpha-p-2 q-1\right)} .
$$

For $m \in \mathbb{N}$, we let $I(m)$ denote the set consisting of the $m$ smallest non-negative integers, i.e.,

$$
I(m)=\{1, \ldots, m\} \subset \mathbb{N} .
$$

Given any subset $J \subseteq \mathbb{N}$, and corresponding sequence $\epsilon(J)=\left\{\epsilon_{j}\right\}_{j \in J}$ of signs $\epsilon_{j}= \pm 1, j \in J$, we let $\lambda+e_{\epsilon(J)}$ denote the sequence defined by

$$
\left(\lambda+e_{\epsilon(J)}\right)_{i}=\lambda_{i}+\epsilon_{i}, \quad i \in \mathbb{N}
$$

where we have set $\epsilon_{i}=0$ if $i \notin J$. With this notation in mind, we are now ready to state the recurrence relations for the Jacobi symmetric functions in a form that is convenient for our purposes.

Theorem 4.15. Let $J \subseteq I \subset \mathbb{N}$ be two finite subsets of the set of (positive) natural numbers $\mathbb{N}$, and fix a sequence $\epsilon(J)=\left\{\epsilon_{j}\right\}_{j \in J}$ of signs $\epsilon_{j}= \pm 1, j \in J$. Introduce the rational function

$$
R_{\epsilon(J)}(z ; m)=\prod_{j \in J} \frac{\left(\epsilon_{j} z_{j}+z_{m}+1 / \alpha\right)\left(\epsilon_{j} z_{j}+p / 2+q+1 / \alpha\right)}{\left(\epsilon_{j} z_{j}-p / 2-q\right)\left(\epsilon_{j} z_{j}-z_{m}\right)} .
$$

Let, furthermore,

$$
\hat{v}^{J}(z)=\frac{z+1 / \alpha}{z}, \quad \hat{w}^{J}(z)=\frac{(z-p / 2-q)(z+(1-p) / 2)}{z(z+1 / 2)},
$$


and introduce the following two rational functions:

$$
\begin{aligned}
\hat{V}_{I, \epsilon(J)}^{(+)}(z)= & \prod_{j \in J} \hat{w}^{J}\left(\epsilon_{j} z_{j}\right) \prod_{\substack{j, j^{\prime} \in J \\
j<j^{\prime}}} \hat{v}^{J}\left(\epsilon_{j} z_{j}+\epsilon_{j^{\prime}} z_{j^{\prime}}\right) \hat{v}^{J}\left(\epsilon_{j} z_{j}+\epsilon_{j^{\prime}} z_{j^{\prime}}+1\right) \\
& \times \prod_{\substack{j \in J \\
i \in I \backslash J}} \hat{v}^{J}\left(\epsilon_{j} z_{j}+z_{i}\right) \hat{v}^{J}\left(\epsilon_{j} z_{j}-z_{i}\right), \\
\hat{V}_{I, \epsilon(J)}^{(-)}(z)= & \prod_{j \in J} \hat{w}^{J}\left(\epsilon_{j} z_{j}\right) \prod_{\substack{j, j^{\prime} \in J \\
j<j^{\prime}}} \hat{v}^{J}\left(\epsilon_{j} z_{j}+\epsilon_{j^{\prime}} z_{j^{\prime}}\right) \hat{v}^{J}\left(-\epsilon_{j} z_{j}-\epsilon_{j^{\prime}} z_{j^{\prime}}-1\right) \\
& \times \prod_{\substack{j \in J \\
i \in I \backslash J}} \hat{v}^{J}\left(\epsilon_{j} z_{j}+z_{i}\right) \hat{v}^{J}\left(\epsilon_{j} z_{j}-z_{i}\right) .
\end{aligned}
$$

To each $z \in \mathbb{F}$, associate the sequence

$$
\rho^{J}(z)=\{(z-i) / \alpha-p / 2-q\}_{i \in \mathbb{N}} .
$$

For each $r \in \mathbb{N}$, let

$$
E_{r}=2^{r} m_{\left(1^{r}\right)},
$$

that is, $E_{r}$ is equal to $2^{r}$ times the rth elementary symmetric function. Then, the re-normalised Jacobi symmetric functions $\mathcal{J}_{\lambda} / \epsilon_{0}\left(\mathcal{J}_{0}\right)$ satisfy, for generic values of $p_{0}$, the recurrence relations

$$
\begin{aligned}
E_{r} \frac{\mathcal{J}_{\lambda}}{\epsilon_{0}\left(\mathcal{J}_{\lambda}\right)}= & \sum_{\epsilon(J), \epsilon(K)}(-1)^{|K|} \hat{V}_{I(\ell(\lambda)+r), \epsilon(J)}^{(+)}\left(\rho^{J}\left(p_{0}\right)+\lambda\right) \hat{V}_{I(\ell(\lambda)+r) \backslash J, \epsilon(K)}^{(-)}\left(\rho^{J}\left(p_{0}\right)+\lambda\right) \\
& \times R_{\epsilon(J)}\left(\rho^{J}\left(p_{0}\right)+\lambda ; \ell(\lambda)+r+1\right) R_{\epsilon(K)}\left(\rho^{J}\left(p_{0}\right)+\lambda ; \ell(\lambda)+r+1\right) \frac{\mathcal{J}_{\lambda+e_{\epsilon(J)}}}{\epsilon_{0}\left(\mathcal{J}_{\lambda+e_{\epsilon(J)}}\right)},
\end{aligned}
$$

where the sum extends over all sequences of signs $\epsilon(J)$ and $\epsilon(K)$ with $J, K \subset I(\ell(\lambda)+r)$ such that $J \cap K=\varnothing,|J|+|K|=r$, and $\lambda+e_{\epsilon(J)}$ is a partition.

Remark 4.16. It is clear from the representation (B.9) that $\mathcal{J}_{\lambda}$, and thereby also $\epsilon_{p_{0}}\left(\mathcal{J}_{\lambda}\right)$, is a rational function of $p_{0}$; c.f., (4.17) and (4.18). It follows that $\epsilon_{p_{0}}\left(\mathcal{J}_{\lambda}\right) \neq 0$ on a dense (open) set in the Zariski topology. It is for these 'generic' values of $p_{0}$ that the recurrence relations (4.24) are valid.

Remark 4.17. As discussed above, Theorem 4.15 is the infinite-dimensional generalisation of a result of van Diejen [47, Theorem 6.4] and is essentially due to Sergeev and Veselov [41] (see Theorem 4.4) - with the difference that the latter authors did not provide an explicit formula for the coefficients in (4.24). We shall require this explicit information in order to obtain corresponding recurrence relations for the Hermite symmetric functions. For the convenience of the reader, we have included a full proof of Theorem 4.15 in Appendix C, expanding on the proof of Theorem 4.4 in Sergeev and Veselov [41].

It is important to note that we can not just simply apply $\sigma_{(-q)^{-1 / 2}} \circ t_{-1}$ to (4.24), and then take the limit $q \rightarrow \infty$, as in Proposition 4.11. Indeed, for $r>1$, the symmetric function $(-q)^{(\ell(\lambda)+r) / 2}\left(\sigma_{(-q)^{-1 / 2}} \circ t_{-1}\right)\left(E_{r} \mathcal{J}_{\lambda}\left(\alpha, p_{0}, p, q\right)\right)$ contains terms which diverge as $q \rightarrow \infty$. However, this problem can be resolved by considering instead appropriate linear combinations of the recurrence relations (4.24). For example, if we are interested in the case $r=2$, then we should observe that

$$
t_{-1}\left(E_{2}+2\left(p_{0}-1\right) E_{1}+2 p_{0}\left(p_{0}-1\right)\right)=E_{2},
$$


and consider the corresponding linear combination of recurrence relations (4.24). For a detailed discussion of this point, in the context of a finite number of variables, see van Diejen [46].

Another issue, which is one of convenience rather than necessity, is the choice of normalisation of the Hermite symmetric functions. In order to find the normalisation for which the corresponding recurrence relations take the simplest possible form, we note the $q \rightarrow \infty$ limit of the normalisation factors $\epsilon_{0}\left(\mathcal{J}_{\lambda}\right)$ :

$$
\lim _{q \rightarrow \infty} \epsilon_{0}\left(\mathcal{J}_{\lambda}\left(\alpha, p_{0}, p, q\right)\right)=\frac{C_{\lambda}^{0}\left(p_{0} / \alpha\right)}{C_{\lambda}^{-}(1 / \alpha)}=\epsilon_{p_{0}}\left(P_{\lambda}\right),
$$

where the second equality follows from a direct comparison of $(2.10)$ and $(4.21 b),(4.21 \mathrm{c})$. As will become clear below, it will be convenient to extract from this limit the factor $C_{\lambda}^{0}\left(p_{0} / \alpha\right)$, which contains all the dependence on the parameter $p_{0}$, and re-normalise the Hermite symmetric functions by the factor $C_{\lambda}^{-}(1 / \alpha)$ only.

We shall make use of the following notation: given a subset $J \subset \mathbb{N}$, and a corresponding sequence of signs $\epsilon(J)$, we shall write $J_{+}$and $J_{-}$for the subsets of $J$ given by

$$
J_{+}=\left\{j \in J: \epsilon_{j}=+1\right\}, \quad J_{-}=\left\{j \in J: \epsilon_{j}=-1\right\} .
$$

With the above remarks in mind, we continue by stating and proving the analogy of Theorem 4.15 for the Hermite symmetric functions.

Proposition 4.18. The re-normalised Hermite symmetric functions

$$
\mathcal{H}_{\lambda}:=C_{\lambda}^{-}(1 / \alpha) H_{\lambda}
$$

satisfy recurrence relations of the form

$$
e_{r} \mathcal{H}_{\lambda}=\sum_{J_{+}, J_{-}} \hat{W}_{I(\ell(\lambda)+r) ; J_{+}, J_{-}}(\lambda) \mathcal{H}_{\lambda+e_{J_{+}}-e_{J_{-}}},
$$

where the sum is over all subsets $J_{+}, J_{-} \subset \mathbb{N}$ such that $J_{+} \cap J_{-}=\varnothing,\left|J_{+}\right|+\left|J_{-}\right| \leq r, r-\left|J_{+}\right|-\left|J_{-}\right|$ is even, and $\lambda+e_{J_{+}}-e_{J_{-}}$is a partition.

Moreover, the coefficients $\hat{W}_{I(\ell(\lambda)+r) ; J_{+}, J_{-}}$are of the form

$$
\hat{W}_{I(\ell(\lambda)+r) ; J_{+}, J_{-}}(\lambda)=\frac{1}{2^{\mid J_{-}}} \prod_{j \in J_{-}}\left(\frac{p_{0}-j+1}{\alpha}+\lambda_{j}-1\right) \hat{U}_{I(\ell(\lambda)+r) ; J_{+}, J_{-}}(\lambda),
$$

where $U_{I(\ell(\lambda)+r) ; J_{+}, J_{-}}$is a polynomial in $p_{0}$, and if $\left|J_{+}\right|+\left|J_{-}\right|=r$, then

$$
\begin{aligned}
\hat{U}_{I(\ell(\lambda)+r) ; J_{+}, J_{-}}(\lambda)= & \prod_{j \in J_{+}, j^{\prime} \in J_{-}}\left(1+\frac{1}{j^{\prime}-j+\alpha\left(\lambda_{j}-\lambda_{j^{\prime}}\right)}\right)\left(1+\frac{1}{j^{\prime}-j+\alpha\left(\lambda_{j}-\lambda_{j^{\prime}}+1\right)}\right) \\
& \times \prod_{j \in J_{-}}\left(\ell(\lambda)+r-j+\alpha \lambda_{j}\right) \prod_{i \in I(\ell(\lambda)+r) \backslash J}\left(1+\frac{1}{j-i+\alpha\left(\lambda_{i}-\lambda_{j}\right)}\right)(4.28) \\
& \times \prod_{j \in J_{+}} \frac{1}{\ell(\lambda)+r+1-j+\alpha \lambda_{j}} \prod_{i \in I(\ell(\lambda)+r) \backslash J}\left(1-\frac{1}{j-i+\alpha\left(\lambda_{i}-\lambda_{j}\right)}\right) .
\end{aligned}
$$

Proof. As noted above, for a unique set of coefficients $c_{r}, \ldots, c_{0} \in \Lambda_{\mathbb{F}}$, we have that

$$
t_{-1}\left(c_{r} E_{r}+c_{r-1} E_{r-1}+\cdots+c_{0}\right)=E_{r} .
$$


Consider the corresponding linear combination of recurrence relations (4.24). For the left-hand side of the resulting relation, Proposition 4.11 and (4.25) yield the limit

$$
\lim _{q \rightarrow \infty}(-q)^{(\ell(\lambda)+r) / 2}\left(\sigma_{(-q)^{-1 / 2}} \circ t_{-1}\right)\left(\left(c_{r} E_{r}+c_{r-1} E_{r-1}+\cdots+c_{0}\right) \frac{\mathcal{J}_{\lambda}}{\epsilon_{0}\left(\mathcal{J}_{\lambda}\right)}\right)=E_{r} \frac{H_{\lambda}}{\epsilon_{p_{0}}\left(P_{\lambda}\right)} .
$$

Furthermore, it is clear that the limit of the right-hand side is of the form

$$
2^{r} \sum_{J_{+}, J_{-}} \hat{W}_{I(\ell(\lambda)+r) ; J_{+}, J_{-}}(\lambda) \frac{H_{\lambda+e_{J_{+}}-e_{J_{-}}}}{\epsilon_{p_{0}}\left(P_{\lambda+e_{J_{+}}-e_{J_{-}}}\right)}
$$

with the coefficients $\hat{W}_{I(\ell(\lambda)+r) ; J_{+}, J_{-}}$given by

$$
\begin{aligned}
\lim _{q \rightarrow \infty} & \frac{(-q)^{\left(r-\left|J_{+}\right|+\left|J_{-}\right|\right) / 2}}{2^{r}} \frac{C_{\lambda}^{0}\left(p_{0} / \alpha\right)}{C_{\lambda+e_{J_{+}}-e_{J_{-}}}^{0}\left(p_{0} / \alpha\right)} \sum_{K_{+}, K_{-}}(-1)^{|K|} \\
& \times \hat{V}_{I(\ell(\lambda)+r), \epsilon(J)}^{(+)}\left(\rho^{J}\left(p_{0}\right)+\lambda\right) R_{\epsilon(J)}\left(\rho^{J}\left(p_{0}\right)+\lambda ; \ell(\lambda)+r+1\right) \\
& \times \hat{V}_{I(\ell(\lambda)+r) \backslash J, \epsilon(K)}^{(-)}\left(\rho^{J}\left(p_{0}\right)+\lambda\right) R_{\epsilon(K)}\left(\rho^{J}\left(p_{0}\right)+\lambda ; \ell(\lambda)+r+1\right) .
\end{aligned}
$$

As a direct computation shows, we have that

$$
\lim _{q \rightarrow \infty} R_{\epsilon(L)}\left(\rho^{J}\left(p_{0}\right)+\lambda ; m\right)=\prod_{j \in L_{+}} \frac{p_{0}-j+1+\alpha \lambda_{j}}{m-j+\alpha\left(\lambda_{j}-\lambda_{m}\right)} \prod_{j \in L_{-}} \frac{m-j-1+\alpha\left(\lambda_{j}-\lambda_{m}\right)}{p_{0}-j+\alpha \lambda_{j}},
$$

and that

$$
\lim _{q \rightarrow \infty}(-q)^{\left|L_{-}\right|} \prod_{j \in L} \hat{w}^{J}\left(\epsilon_{j}\left(\rho^{J}\left(p_{0}\right)+\lambda\right)_{j}\right)=2^{\left|L_{-}\right|} \prod_{j \in L_{-}}\left(\frac{p_{0}-j}{\alpha}+\lambda_{j}\right)
$$

for $L=J, K$. We observe that, for all arguments $z$ appearing in (4.24), $\hat{v}(z)$ is a bounded function of $q$; c.f., (4.23). It follows that a given term in (4.29) provides a non-zero contribution only if

$$
\frac{r-\left|J_{+}\right|-\left|J_{-}\right|}{2}-\left|K_{-}\right|=0,
$$

which clearly can hold true only if $r-\left|J_{+}\right|-\left|J_{-}\right|$is even. This concludes the proof of the first part of the statement.

In order to establish the stated structure of the coefficients $\hat{W}_{I(\ell(\lambda)+r) ; J_{+}, J_{-}}$, we observe that

$$
\frac{C_{\lambda}^{0}\left(p_{0} / \alpha\right)}{C_{\lambda+e_{J_{+}}-e_{J_{-}}}^{0}\left(p_{0} / \alpha\right)}=\frac{\prod_{j \in J_{-}}\left(p_{0}-j+1+\alpha\left(\lambda_{j}-1\right)\right)}{\prod_{j \in J_{+}}\left(p_{0}-j+1+\alpha \lambda_{j}\right)} .
$$

If we now set $m=\ell(\lambda)+r+1$ (c.f., (4.24)), and combine the observations made thus far, we readily deduce (4.27). Moreover, in case $\left|J_{+}\right|+\left|J_{-}\right|=r$, we have $K=\emptyset$. It follows that the sum in (4.29) contains only one term, and a direct computation yields (4.28).

Remark 4.19. From the representation (B.9) we can directly infer that $H_{\lambda}$, and thereby also $\mathcal{H}_{\lambda}$, is a polynomial in $p_{0}$; c.f., Definition 4.1. In contrast to the Jacobi case, this entails that the recurrence relations (4.26) are valid not only for generic but indeed all values of the parameter $p_{0}$. 
If we restrict our attention to $r=1$, then the statement can be simplified considerably. In particular, all coefficients can be specified explicitly.

Corollary 4.20. The re-normalised Hermite symmetric functions $\mathcal{H}_{\lambda}$ satisfy the recurrence relation

$$
e_{1} \mathcal{H}_{\lambda}=\sum_{j=1}^{\ell(\lambda)+1}\left(\hat{W}_{j}(\lambda) \mathcal{H}_{\lambda+e_{j}}+\hat{W}_{-j}(\lambda) \mathcal{H}_{\lambda-e_{j}}\right)
$$

with the coefficients

$$
\begin{aligned}
\hat{W}_{j}(\lambda)= & \frac{1}{\ell(\lambda)+2-j+\alpha \lambda_{j}} \prod_{\substack{1 \leq i \leq \ell(\lambda)+1 \\
i \neq j}}\left(1-\frac{1}{j-i+\alpha\left(\lambda_{i}-\lambda_{j}\right)}\right), \\
\hat{W}_{-j}(\lambda)= & \frac{1}{2}\left(\frac{p_{0}-j+1}{\alpha}+\lambda_{j}-1\right)\left(\ell(\lambda)+1-j+\alpha \lambda_{j}\right) \\
& \times \prod_{\substack{1 \leq i \leq \ell(\lambda)+1 \\
i \neq j}}\left(1+\frac{1}{j-i+\alpha\left(\lambda_{i}-\lambda_{j}\right)}\right) .
\end{aligned}
$$

Remark 4.21. When restricted to the polynomial case, Corollary 4.20 and Proposition 4.27 respectively reduce to Propositions 2.5 and 2.6 of [46]. The finite-dimensional analogue of Corollary 4.20 can also be found in [2, Proposition 3.5].

We proceed to consider how the recurrence relation (4.31) is related to the question of existence of invariant ideals. To this end, let $I \subset \Lambda_{\mathbb{F}}$ be an ideal invariant under the differential operators $\mathcal{L}_{f}^{H}, f \in \Lambda_{\mathbb{F}, \alpha}$. By Lemma 4.13, there exists at least one partition $\lambda$ such that $H_{\lambda} \in I$. In case $\lambda=(0)$, we have that $H_{\lambda}=1$, and consequently that $I=\Lambda_{\mathbb{F}}$. Suppose that $\lambda \neq 0$. Then, we can always find an integer $j=1, \ldots,|\lambda|$ such that $\lambda-e_{j}$ is a partition. For example, $j=|\lambda|$. It is clear from Corollary 4.20 that

$$
\hat{W}_{-j}(\lambda)=0
$$

if and only if

$$
p_{0}=j-1-\alpha\left(\lambda_{j}-1\right) .
$$

Hence, if $p_{0}$ is not of this form, then we can conclude that also $H_{\lambda-e_{j}} \in I$. Moreover, assuming this to be the case for all partitions $\mu \subset \lambda$, it follows by induction on the weight $|\lambda|$ of $\lambda$ that again $1 \in I$. This observation forms one part of the main result of this section, as stated in the following theorem:

Theorem 4.22. $\Lambda_{\mathbb{F}}$ contains a non-trivial ideal invariant under all differential operators $\mathcal{L}_{f}^{H}$, $f \in \Lambda_{\mathbb{F}, \alpha}$, for and only for non-zero $p_{0}$ of the form

$$
p_{0}=n-\alpha m, \quad n, m \in \mathbb{N}_{0} .
$$

If that is the case, then there is a unique such ideal, spanned by the Hermite symmetric functions $H_{\lambda}$ labelled by the partitions $\lambda$ such that $(n+1, m+1) \in \lambda$.

Proof. There remains only to prove that, for $p_{0}=n-\alpha m$, there exists a unique non-trivial invariant ideal

$$
I=\mathbb{F}\left\langle H_{\lambda}:(n+1, m+1) \in \lambda\right\rangle \subset \Lambda_{\mathbb{F}} .
$$


Suppose that a partition $\lambda$ and subsets $J_{+}, J_{-} \subset \mathbb{N}$ are such that $(n+1, m+1) \in \lambda$ but $(n+1, m+1) \notin \lambda+e_{J_{+}}-e_{J_{-}}$. Clearly, this is only possible if $\lambda_{n+1}=m+1$, and $n+1 \in J_{-}$. Fix $r \in \mathbb{N}$, and consider the corresponding recurrence relation (4.26). Since $\hat{W}_{I(\ell(\lambda)+r) ; J_{+}, J_{-}}$is a polynomial in $p_{0}$, it follows from (4.27) that

$$
\hat{W}_{I(\ell(\lambda)+r) ; J_{+}, J_{-}}(\lambda)=0 .
$$

Hence, $I$ is indeed an ideal, which, by construction, is invariant. In order to establish uniqueness, we let $I^{\prime} \subset \Lambda_{\mathbb{F}}$ be any non-trivial invariant ideal. Suppose that $H_{\lambda} \in I^{\prime}$ for some partition $\lambda$ such that $(n+1, m+1) \notin \lambda$. Then, by following the discussion preceding the Theorem, we obtain that $1 \in I^{\prime}$. We must therefore have $I^{\prime} \subseteq I$. In addition, we can exclude the possibility that $I^{\prime} \neq I$ by observing that, starting from any $\mathcal{H}_{\lambda} \in I$, we can obtain any other Hermite symmetric function $\mathcal{H}_{\mu}$ by applying the recurrence relation (4.31).

\section{$5 \quad$ Laguerre symmetric functions}

In this section we shall introduce and study Laguerre symmetric functions as eigenfunctions of the differential operator

$$
\mathcal{L}^{L}=D^{1}+(a+1) E^{0}-\nu E^{1}
$$

with $a$ an indeterminate, and $\nu \in \mathbf{F}$. We shall follow closely our treatment of the Hermite symmetric functions in Section 4. To avoid unnecessary repetitions, the discussion will therefore be brief, and statements that can be obtained as straightforward generalisations from the Hermite case will be stated without proofs.

It is clear from Lemma B.4 that

$$
\mathcal{L}^{L} P_{\lambda}=-\nu|\lambda| P_{\lambda}+\sum_{\mu \subset \lambda} c_{\lambda \mu} P_{\mu}
$$

for some coefficients $c_{\lambda \mu} \in \mathbf{K}$; c.f., (2.6). Theorem B.6 thus guarantees that we can make the following definition:

Definition 5.1. Let $\lambda$ be a partition. We then define the Laguerre symmetric function $L_{\lambda}\left(\alpha, p_{0}, a, \nu\right)$ as the unique symmetric function such that

1. $L_{\lambda}=P_{\lambda}+\sum_{\mu \subset \lambda} u_{\lambda \mu} P_{\mu}$ for some $u_{\lambda \mu} \in \mathbf{K}$,

2. $\mathcal{L}^{L} L_{\lambda}=-\nu|\lambda| L_{\lambda}$.

Following the proof of Proposition 4.3, we can establish a constructive definition.

Proposition 5.2. Let $\lambda$ be a partition and set $L=|\lambda|$. Then, we have that

$$
L_{\lambda}=\exp _{L}\left(-\frac{1}{\nu}\left(D^{1}+(a+1) E^{0}\right)\right)\left(P_{\lambda}\right) .
$$

\subsection{A symmetry property}

Here, we obtain a symmetry property of the Laguerre symmetric functions that does not have a counterpart in the case of symmetric polynomials. This fact follows easily once we have shown that the second-order CMS operator (5.1) posess the symmetry in question.

We write $D^{1}\left(\alpha, p_{0}\right)$ and $E^{0}\left(p_{0}\right)$ for the operators $D^{1}$ and $E^{0}$ of Definition 2.3 , and let

$$
a^{\prime}=2 / \alpha-a-2, \quad p_{0}^{\prime}=p_{0}-1+\alpha(a+1) .
$$


Then, by expanding the series defining $D^{1}\left(\alpha, p_{0}^{\prime}\right)$ and $E^{0}\left(\alpha, p_{0}^{\prime}\right)$ and concentrating on the terms depending on $p_{0}$, one readily verifies that

$$
\begin{aligned}
& D^{1}\left(\alpha, p_{0}^{\prime}\right)=D^{1}\left(\alpha, p_{0}\right)+\frac{2}{\alpha}(\alpha(a+1)-1) E^{0}\left(p_{0}\right)+\frac{1}{\alpha}(\alpha(a+1)-1)(\alpha(a+1)-2) \partial\left(p_{1}\right), \\
& E^{0}\left(p_{0}^{\prime}\right)=E^{0}\left(p_{0}\right)+(\alpha(a+1)-1) \partial\left(p_{1}\right) .
\end{aligned}
$$

These equations, together with the independence of $E^{1}$ upon $p_{0}$, imply the following symmetry property:

$$
D^{1}\left(\alpha, p_{0}^{\prime}\right)+\left(a^{\prime}+1\right) E^{0}\left(p_{0}^{\prime}\right)-\nu E^{1}=D^{1}\left(\alpha, p_{0}\right)+(a+1) E^{0}\left(p_{0}\right)-\nu E^{1} .
$$

Finally, a direct comparison of this equation with Definition 5.1 or Proposition 5.2 establishes the desired symmetry relation.

Proposition 5.3. Let $a^{\prime}$ and $p_{0}^{\prime}$ be as in (5.3). Then, we have that

$$
L_{\lambda}\left(\alpha, p_{0}^{\prime}, a^{\prime}, \nu\right)=L_{\lambda}\left(\alpha, p_{0}, a, \nu\right) .
$$

\subsection{A duality relation}

We continue by considering the action of the homomorphism $\omega_{\alpha}$ on the Laguerre symmetric functions; c.f., (4.5). Starting from the representation (5.2), a direct application of Lemma 4.4 yields

$$
\omega_{\alpha}\left(L_{\lambda}\left(\alpha, p_{0}, a, \nu\right)\right)=\exp _{L}\left(-\frac{1}{\alpha \nu}\left(D^{1}\left(1 / \alpha,-\alpha p_{0}\right)+(1-\alpha a) E^{0}\right)\right)\left(Q_{\lambda^{\prime}}(1 / \alpha)\right) .
$$

We thus arrive at the following proposition:

Proposition 5.4. We have that

$$
\omega_{\alpha}\left(L_{\lambda}\left(\alpha, p_{0}, a, \nu\right)\right)=b_{\lambda^{\prime}}(1 / \alpha) L_{\lambda^{\prime}}\left(1 / \alpha,-\alpha p_{0},-\alpha a,-\alpha \nu\right) .
$$

For the remainder of this section, we shall assume that $\nu=1$. This parameter can be reintroduced by using the fact that

$$
\left(D^{1}+(a+1) E^{0}-\nu E^{1}\right) \circ \sigma_{\nu}=\nu \sigma_{\nu} \circ\left(D^{1}+(a+1) E^{0}-E^{1}\right) ;
$$

c.f., (3.4). In particular, this intertwining relation implies that

$$
L_{\lambda}\left(\alpha, p_{0}, a, \nu\right)=\nu^{-|\lambda|} \sigma_{\nu}\left(L_{\lambda}\left(\alpha, p_{0}, a, 1\right)\right) .
$$

\subsection{A generating function}

Proceeding as in the proof of Proposition 4.6, but using Proposition 4.1 rather than 3.1 in Baker and Forrester [2], it is straightforward to verify the generating function expansion given in the following proposition:

Proposition 5.5. Let $q=1+\left(p_{0}-1\right) / \alpha$. Then, we have that

$$
\sum_{\lambda} \frac{1}{h_{\lambda}[a+q]_{\lambda}} \frac{L_{\lambda} \otimes P_{\lambda}}{\epsilon_{p_{0}}\left(P_{\lambda}\right)}={ }_{0} \mathscr{F}_{1}(a+q) e^{-1 \otimes p_{1}} .
$$


As detailed in Section 4.2 in the case of the Hermite symmetric functions (see the paragraph containing (4.15)), by exploiting the Baker-Campbell-Hausdorff formula, we can deduce an infinite-dimensional family of eigenoperators for the Laguerre symmetric functions, parameterised by the shifted symmetric functions.

Proposition 5.6. Fix $f \in \Lambda_{\mathbb{F}, \alpha}$, and let $k$ be the degree of $f$. Let, furthermore,

$$
\begin{aligned}
\mathcal{L}_{f}^{L}= & \mathcal{L}_{f}-\left[D^{1}+(a+1) E^{0}, \mathcal{L}_{f}\right]+\frac{1}{2 !}\left[D^{1}+(a+1) E^{0},\left[D^{1}+(a+1) E^{0}, \mathcal{L}_{f}\right]\right] \\
& \left.+\cdots+\frac{(-1)^{k}}{k !}\left[D^{1}+(a+1) E^{0}\right), \ldots,\left[D^{1}+(a+1) E^{0}, \mathcal{L}_{f}\right] \cdots\right] .
\end{aligned}
$$

Then, $\mathcal{L}^{L}$ is a differential operator on $\Lambda_{\mathbf{K}}$ of order $2 k$. Moreover, it is the unique operator on $\Lambda_{\mathbf{K}}$ such that

$$
\mathcal{L}_{f}^{L} L_{\lambda}=f(\lambda) L_{\lambda}
$$

for all partitions $\lambda$.

\subsection{Limit transition from the Laguerre to the Hermite symmetric functions}

As is well known for the one-variable case (see for instance [20, Section 2.22]), there is a simple limit transition from the Laguerre polynomials to the Hermite polynomials. Explicitly,

$$
\lim _{a \rightarrow \infty}\left(\frac{2}{a}\right)^{\frac{n}{2}} L^{a}(\sqrt{2 a} x+a)=\frac{(-1)^{n}}{n !} H_{n}(x),
$$

where $L_{n}$ and $H_{n}$ respectively denote the standard (and consequently, non-monic) Laguere and Hermite polynomials.

Computer simulations suggest that a very similar transition relation holds between the Laguerre and Hermite symmetric functions. It seems that the corresponding transition for any finite number of variables greater than one, has been overlooked by the authors cited in the list of references.

Conjecture. For any partition $\lambda$ and generic values of the parameters $\alpha$ and $\nu$, we have

$$
\lim _{a \rightarrow \infty}\left(\frac{1}{2 a}\right)^{\frac{|\lambda|}{2}} \sigma_{\sqrt{2 a}} \circ t_{a / \nu}\left(L_{\lambda}(\alpha, a, \nu)\right)=H_{\lambda}\left(\alpha, \nu^{2}\right) .
$$

One easily checks that the translation and the scale change induced by $\sigma_{\sqrt{2 a}} \circ t_{a / \nu}$ allow to map, as $a \rightarrow \infty$, the Laguerre operator $\mathcal{L}^{L}$ and the Hermite operator $\mathcal{L}^{H}$. This is not sufficient however for proving the conjecture. The difficulty resides in the fact that the Laguerre symmetric function $L_{\Lambda}(\alpha, a, \nu)$ has degree $|\lambda|$ in the parameter $a$, while $t_{a / \nu} L_{\Lambda}(\alpha, a, \nu)$ appears to have degree $|\lambda| / 2$. Proving the latter property would require some new identities about the generalised binomial coefficients.

\subsection{A limit from the Jacobi symmetric functions}

Also the Laguerre symmetric functions $L_{\lambda}\left(\alpha, p_{0}, a\right)$ can be viewed as limits of the Jacobi symmetric functions $\mathcal{J}_{\lambda}\left(\alpha, p_{0}, p, q\right)$. To make this precise, we set $a=-p-q-1 / 2$, and observe that

$$
q^{-1} \sigma_{2 q^{-1}} \circ \mathcal{L}^{J}=\left(D^{1}+(a+1) E^{0}-E^{1}\right) \circ \sigma_{2 q^{-1}}+q^{-1}\left(D^{2}+(a+3 / 2) E^{1}\right) \circ \sigma_{2 q^{-1}} .
$$

From the representation (B.9) we can thus infer the following proposition: 
Proposition 5.7. Let $\lambda$ be a partition. Then, for generic parameter values, we have that

$$
L_{\lambda}\left(\alpha, p_{0}, a\right)=\lim _{q \rightarrow \infty}(q / 2)^{|\lambda|} \sigma_{2 q^{-1}}\left(\mathcal{J}_{\lambda}\left(\alpha, p_{0},-a-q-1 / 2, q\right)\right)
$$

in the sense of term-wise convergence.

\subsection{Structure of Pieri formulae and invariant ideals}

The Laguerre case is in many ways easier to handle than the Hermite case. The reason being that, if we apply $\sigma_{2 q^{-1}}$ to (4.24), then the limit $q \rightarrow \infty$ is well-defined. By Proposition 5.7, this directly yields Pieri type recurrence relations for the Laguerre symmetric functions.

We first note the appropriate limit of the normalisation factors $\epsilon_{0}\left(\mathcal{J}_{\lambda}\right)$ :

$$
\lim _{q \rightarrow \infty}(-q / 2)^{|\lambda|} \epsilon_{0}\left(\mathcal{J}_{\lambda}\left(\alpha, p_{0},-q-a-1 / 2, q\right)\right)=\frac{C_{\lambda}^{0}\left(p_{0} / \alpha\right) C_{\lambda}^{0}\left(\left(p_{0}-1\right) / \alpha+a+1\right)}{C_{\lambda}^{-}(1 / \alpha)} .
$$

In particular, this means that the left-hand side of the recurrence relations (4.24) for the Jacobi symmetric functions have the limits

$$
\lim _{q \rightarrow \infty}\left(\sigma_{2 q^{-1}}\right)\left(\frac{\mathcal{J}_{\lambda}\left(\alpha, p_{0},-q-a-1 / 2, q\right)}{\epsilon_{0}\left(\mathcal{J}_{\lambda}\left(\alpha, p_{0},-q-a-1 / 2, q\right)\right)}\right)=(-1)^{|\lambda|} \frac{\mathcal{L}_{\lambda}\left(\alpha, p_{0}, a\right)}{C_{\lambda}^{0}\left(p_{0} / \alpha\right) C_{\lambda}^{0}\left(\left(p_{0}-1\right) / \alpha+a+1\right)}
$$

where we have introduced the re-normalised Laguerre symmetric functions

$$
\mathcal{L}_{\lambda}\left(\alpha, p_{0}, a\right)=C_{\lambda}^{-}(1 / \alpha) L_{\lambda}\left(\alpha, p_{0}, a\right)
$$

It is straightforward to verify that the $q \rightarrow \infty$ limit of $R_{\epsilon(L)}\left(\rho^{J}\left(p_{0}\right)+\lambda\right)$ is, just as in the Hermite case, given by (4.30), while

$$
\lim _{q \rightarrow \infty}(-q)^{|L|} \prod_{j \in L} \hat{w}^{J}\left(\epsilon_{j}\left(\rho^{J}\left(p_{0}\right)+\lambda\right)_{j}\right)=4^{|L|} \prod_{j \in L_{+}}\left(\frac{p_{0}-j}{\alpha}+\lambda_{j}+a+1\right) \prod_{j \in L_{-}}\left(\frac{p_{0}-j}{\alpha}+\lambda_{j}\right)
$$

for $L=J, K$. In addition, we have that

$$
\begin{gathered}
\frac{C_{\lambda}^{0}\left(p_{0} / \alpha\right) C_{\lambda}^{0}\left(\left(p_{0}-1\right) / \alpha+a+1\right)}{C_{\lambda+e_{J_{+}}-e_{J_{-}}}^{0}\left(p_{0} / \alpha\right) C_{\lambda+e_{J_{+}}-e_{J_{-}}}^{0}\left(\left(p_{0}-1\right) / \alpha+a+1\right)} \\
=\frac{\prod_{j \in J_{-}}\left(p_{0}-j+1+\alpha\left(\lambda_{j}-1\right)\right)\left(p_{0}-j+\alpha\left(\lambda_{j}+a\right)\right)}{\prod_{j \in J_{+}}\left(p_{0}-j+1+\alpha \lambda_{j}\right)\left(p_{0}-j+\alpha\left(\lambda_{j}+1+a\right)\right)} .
\end{gathered}
$$

Now, proceeding in analogy with the proof of Proposition 4.18, we arrive at the corresponding recurrence relations for the re-normalised Laguerre symmetric functions.

Proposition 5.8. The re-normalised Laguerre symmetric functions $\mathcal{L}_{\lambda}$ satisfy recurrence relations of the form

$$
(-1 / 2)^{r} e_{r} \mathcal{L}_{\lambda}=\sum_{J_{+}, J_{-}} \hat{W}_{I(\ell(\lambda)+r) ; J_{+}, J_{-}}(\lambda) \mathcal{L}_{\lambda+e_{J_{+}}-e_{J_{-}}},
$$

where the sum extends over all subsets $J_{+}, J_{-} \subset \mathbb{N}$ such that $J_{+} \cap J_{-}=\varnothing,\left|J_{+}\right|+\left|J_{-}\right| \leq r$, and $\lambda+e_{J_{+}}-e_{J_{-}}$is a partition. 
Moreover, the coefficients $\hat{W}_{I(\ell(\lambda)+r) ; J_{+}, J_{-}}$are given explicitly by

$$
\hat{W}_{I(\ell(\lambda)+r) ; J_{+}, J_{-}}(\lambda)=(-1)^{r-p} \hat{V}_{I(\ell(\lambda)+r) ; J_{+}, J_{-}}(\lambda) \sum_{K_{+}, K_{-}} \hat{V}_{I(\ell(\lambda)+r) \backslash\left(J_{+} \cup J_{-}\right) ; K_{+}, K_{-}}(\lambda)
$$

with

$$
\begin{aligned}
& \hat{V}_{I(\ell(\lambda)+r) ; J_{+}, J_{-}}(\lambda)=\prod_{j \in J_{-}}\left(\frac{p_{0}-j+1}{\alpha}+\lambda_{j}-1\right)\left(\frac{p_{0}-j}{\alpha}+\lambda_{j}+a\right) \\
& \quad \times \prod_{j \in J_{+}, j^{\prime} \in J_{-}}\left(1+\frac{1}{j^{\prime}-j+\alpha\left(\lambda_{j}-\lambda_{j^{\prime}}\right)}\right)\left(1+\frac{1}{j^{\prime}-j+\alpha\left(\lambda_{j}-\lambda_{j^{\prime}}+1\right)}\right) \\
& \quad \times \prod_{j \in J_{-}}\left(\ell(\lambda)+r-j+\alpha \lambda_{j}\right) \prod_{i \in I(\ell(\lambda)+r) \backslash J}\left(1+\frac{1}{j-i+\alpha\left(\lambda_{i}-\lambda_{j}\right)}\right) \\
& \quad \times \prod_{j \in J_{+}} \frac{1}{\ell(\lambda)+r+1-j+\alpha \lambda_{j}} \prod_{i \in I(\ell(\lambda)+r) \backslash J}\left(1-\frac{1}{j-i+\alpha\left(\lambda_{i}-\lambda_{j}\right)}\right),
\end{aligned}
$$

and where the sum runs over all subsets $K_{+}, K_{-} \subset I(\ell(\lambda)+r) \backslash\left(J_{+} \cup J_{-}\right)$such that $K_{+} \cap K_{-}=\varnothing$ and $\left|K_{+}\right|+\left|K_{-}\right|=r-\left|J_{+}\right|-\left|J_{-}\right|$.

As a direct consequence, we have the following corollary:

Corollary 5.9. The re-normalised Laguerre symmetric functions $\mathcal{L}_{\lambda}$ satisfy the recurrence relation

$$
(-1 / 2) e_{1} \mathcal{L}_{\lambda}=\sum_{j=1}^{|\lambda|+1}\left(\hat{W}_{j}(\lambda) \mathcal{L}_{\lambda+e_{j}}+\hat{W}_{-j}(\lambda) \mathcal{L}_{\lambda-e_{j}}\right)
$$

with the coefficients

$$
\begin{aligned}
\hat{W}_{j}(\lambda)= & \frac{1}{\ell(\lambda)+2-j+\alpha \lambda_{j}} \prod_{\substack{1 \leq i \leq|\lambda|+1 \\
i \neq j}}\left(1-\frac{1}{j-i+\alpha\left(\lambda_{i}-\lambda_{j}\right)}\right), \\
\hat{W}_{-j}(\lambda)= & \left(\frac{p_{0}-j+1}{\alpha}+\lambda_{j}-1\right)\left(\frac{p_{0}-j}{\alpha}+\lambda_{j}+a\right)\left(\ell(\lambda)+1-j+\alpha \lambda_{j}\right) \\
& \times \prod_{\substack{1 \leq i \leq|\lambda|+1 \\
i \neq j}}\left(1+\frac{1}{j-i+\alpha\left(\lambda_{i}-\lambda_{j}\right)}\right) .
\end{aligned}
$$

Remark 5.10. Corollary 5.9 generalises results that were known in the polynomial case, namely Proposition 4.7 of [2]. Similarly, by restricting the symmetric functions in Proposition 5.8 to a finite number of variables, one recovers Propositions 2.6 and 2.7 of [46].

It is a straightforward exercise to adapt the discussion succeeding Corollary 4.20, as well as the proof of Theorem 4.22, to the Laguerre case. In this way, one arrives at the main result of this section.

Theorem 5.11. $\Lambda_{\mathbb{F}}$ contains a non-trivial ideal invariant under all differential operators $\mathcal{L}_{f}^{L}$, $f \in \Lambda_{\mathbb{F}, \alpha}$, for and only for non-zero $p_{0}$ of the form

$$
p_{0}=n-\alpha m \quad \text { or } \quad p_{0}=n+1-\alpha(m+a+1), \quad n, m \in \mathbb{N}_{0} .
$$

If that is the case, then there is a unique such ideal, spanned by the Laguerre symmetric functions $L_{\lambda}$ labelled by the partitions $\lambda$ such that $(n+1, m+1) \in \lambda$.

We note that the presence of the second family of ideals can be explained by the symmetry property in Proposition 5.3. Indeed, we have that $p_{0}^{\prime}=n+1-\alpha\left(m+a^{\prime}+1\right)$. 


\section{Deformed CMS operators and super polynomials}

We set the parameter $p_{0}=n-\alpha m$ for some $n, m \in \mathbb{N}_{0}$, and let $I_{n, m} \subset \Lambda_{\mathbb{F}}$ be the subspace given by

$$
I_{n, m}=\mathbb{F}\left\langle P_{\lambda}:(n+1, m+1) \in \lambda\right\rangle .
$$

Although it is not obvious from its definition, $I_{n, m}$ is an ideal in $\Lambda_{\mathbb{F}}$. This fact follows immediately from well-known Pieri formulae for Jack's symmetric functions; see, e.g., Chapter VI in Macdonald [29].

In this section we shall show that any CMS operator of the form (2.18) admits a restriction onto the quotient ring $\Lambda_{\mathbb{F}} / I_{n, m}$. Moreover, these restrictions will be given explicitly by differential operators of so-called deformed CMS type. Deformed CMS operators were first introduced by Chalykh, Feigen, Veselov [6] and later studied by many authors [11, 12, 21, 40, 42, 43]. We shall consider the Hermite and Laguerre cases in some detail. In particular, this restriction procedure will lead to the introduction of super Hermite and super Laguerre polynomials. Furthermore, the results we have obtained in the context of symmetric functions more or less immediately restrict to corresponding results for these 'super' polynomials.

At this point, it is interesting to recall that, in the Hermite and Laguerre cases, we know, by Theorems 4.22 and 5.11, that there exists a unique ideal invariant under the action of all eigenoperators. We can thus conclude that the corresponding restrictions are given precisely by the deformed CMS operators mentioned above. In terms of the ideal $I_{n, m}$ this fact is equivalent to the equalities

$$
I_{n, m}=\mathbb{F}\left\langle H_{\lambda}:(n+1, m+1) \in \lambda\right\rangle=\mathbb{F}\left\langle L_{\lambda}:(n+1, m+1) \in \lambda\right\rangle .
$$

We stress that these equalities are non-trivial. For example, by definition, the Hermite symmetric functions are of the form

$$
H_{\lambda}=P_{\lambda}+\sum_{\mu \subset \lambda} u_{\lambda \mu} P_{\mu}, \quad u_{\lambda \mu} \in \mathbb{F}
$$

for $p_{0}=n-\alpha m$. The former of the above equalities thus implies that the coefficient $u_{\lambda \mu}=0$ for all partitions $\lambda, \mu$ such that $(n+1, m+1) \in \lambda$ and $(n+1, m+1) \notin \mu$.

\subsection{Super Jack polynomials}

Our discussion below will involve a few results from the theory of super Jack polynomials which we now briefly recall. Let $x=\left(x_{1}, \ldots, x_{n}\right)$ and $y=\left(y_{1}, \ldots, y_{m}\right)$ be two sequences of indeterminates, and consider the subalgebra

$$
\Lambda_{\mathbb{F}, n, m} \subset \mathbb{F}\left[x_{1}, \ldots, x_{n}, y_{1}, \ldots, y_{m}\right]
$$

consisting of all polynomials $p(x, y)$ that are separately symmetric in the variables $x$ and $y$, and that satisfy the condition

$$
\left.\left(\frac{\partial p}{\partial x_{i}}+\frac{1}{\alpha} \frac{\partial p}{\partial y_{I}}\right)\right|_{x_{i}=y_{I}}=0
$$

for all $i=1, \ldots, n$ and $I=1, \ldots, m$. This algebra generalises that of so-called supersymmetric polynomials, which were first introduced as characters in the representation theory of the superalgebra $g l(n \mid m)$; see, e.g., Examples 23 and 24 in Section I.3 of Macdonald [29] or Moens 
and Van der Jeugt [30]. As shown by Sergeev and Veselov [42], the algebra $\Lambda_{\mathbb{F}, n, m}$ is generated by the 'deformed' power sums

$$
p_{r, \alpha}(x, y)=\sum_{i=1}^{n} x_{i}^{r}-\alpha \sum_{I=1}^{m} y_{I}^{r}, \quad r \in \mathbb{N} .
$$

This yields a surjective homomorphism $\varphi_{n, m}=\Lambda_{\mathbb{F}} \rightarrow \Lambda_{\mathbb{F}, n, m}$ by

$$
\varphi_{n, m}\left(p_{r}\right)=p_{r, \alpha}(x, y), \quad r \in \mathbb{N} \text {. }
$$

Moreover, the kernel of this homomorphism is known to be spanned by the Jack's symmetric functions $P_{\lambda}$ with $(n+1, m+1) \in \lambda$; see Theorem 2 in [43].

It is clear from the facts listed above that $\varphi_{n, m}$ yields an isomorphism

$$
\varphi_{n, m}: \Lambda_{\mathbb{F}} / I_{n, m} \stackrel{\sim}{\longrightarrow} \Lambda_{\mathbb{F}, n, m},
$$

and that the super Jack polynomials, defined, as in Kerov et al. [19], by

$$
S P_{\lambda}(x, y)=\varphi_{n, m}\left(P_{\lambda}\right)
$$

form a basis for $\Lambda_{\mathbb{F}, n, m}$ as $\lambda$ runs through all partitions such that $(n+1, m+1) \notin \lambda$. It is also clear that $\varphi_{n, m}$ intertwines between eigenoperators of Jack's symmetric functions and super Jack polynomials: for each $f \in \Lambda_{\mathbb{F}, \alpha}$ there exists a unique differential operator $\mathcal{L}_{f, n, m}$ on $\Lambda_{\mathbb{F}, n, m}$ such that the diagram

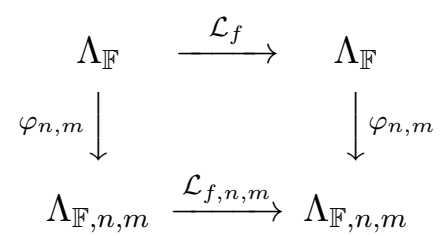

is commutative. Indeed, the differential operators $\mathcal{L}_{f, n, m}$ are given by their action on the super Jack polynomials:

$$
\mathcal{L}_{f, n, m} S P_{\lambda}(x, y)=f(\lambda) S P_{\lambda}(x, y) .
$$

For further details see, in particular, Sergeev and Veselov [43].

We continue by observing that the duality relation (4.6) for Jack's symmetric functions has an interesting analogue for super Jack polynomials. This observation, which is made precise in the proposition below, is essentially due to the fact that

$$
-\alpha p_{r, 1 / \alpha}(y, x)=p_{r, \alpha}(x, y) .
$$

Proposition 6.1. The super Jack polynomials satisfy the duality relation

$$
S P_{\lambda}(\alpha ; x, y)=(-1)^{|\lambda|} S Q_{\lambda^{\prime}}(1 / \alpha ; y, x)
$$

for all partitions $\lambda$ such that $(n+1, m+1) \notin \lambda$, where

$$
S Q_{\lambda}(x, y)=b_{\lambda} S P_{\lambda}(x, y) ;
$$

c.f. (4.7). 
Proof. For clarity of exposition, we shall make explicit the dependence on $\alpha$ by writing $\varphi_{n, m}^{(\alpha)}$ for $\varphi_{n, m}$. In particular, this means that

$$
\varphi_{m, n}^{(1 / \alpha)}\left(p_{r}\right)=p_{r, 1 / \alpha}(y, x), \quad r \in \mathbb{N} .
$$

We proceed to consider the action of the homomorphism $\varphi_{m, n}^{(1 / \alpha)} \circ \omega_{\alpha} \circ \sigma_{-1}$ on one of Jack's symmetric function $P_{\lambda}$. Since $\omega_{\alpha}\left(p_{r}\right)=(-1)^{r-1} \alpha p_{r}$ and $\sigma_{-1}\left(p_{r}\right)=(-1)^{r} p_{r}$, we have that

$$
\left(\varphi_{m, n}^{(1 / \alpha)} \circ \omega_{\alpha} \circ \sigma_{-1}\right)\left(p_{r}\right)=p_{r, \alpha}(x, y), \quad r \in \mathbb{N} .
$$

We can thus conclude that

$$
\varphi_{m, n}^{(1 / \alpha)} \circ \omega_{\alpha} \circ \sigma_{-1}=\varphi_{n, m}^{(\alpha)}
$$

This yields the left-hand side of (6.3). On the other hand, it follows from (4.6), and the fact that $P_{\lambda}$ is homogeneous of degree $|\lambda|$, that

$$
\left(\varphi_{m, n}^{(1 / \alpha)} \circ \omega_{\alpha} \circ \sigma_{-1}\right)\left(P_{\lambda}\right)=(-1)^{|\lambda|} \varphi_{m, n}^{1 / \alpha}\left(Q_{\lambda^{\prime}}(1 / \alpha)\right),
$$

and we arrive at the right-hand side of (6.3).

We conclude this section by showing that the duality relation for the super Jack polynomials implies a similar duality relation for the following 'super' version of the hypergeometric series introduced in (3.8):

$$
{ }_{p} S F_{q}\left(a_{1}, \ldots, a_{p} ; b_{1}, \ldots, b_{q} ; \alpha ; x, y\right)=\sum_{\lambda} \frac{1}{h_{\lambda}} \frac{\left[a_{1}\right]_{\lambda} \cdots\left[a_{p}\right]_{\lambda}}{\left[b_{1}\right]_{\lambda} \cdots\left[b_{q}\right]_{\lambda}} S P_{\lambda}(\alpha ; x, y),
$$

where the sum extends over all partition $\lambda$ such that $(n+1, m+1) \notin \lambda$.

Proposition 6.2. Let

$$
\alpha^{\prime}=1 / \alpha, \quad x^{\prime}=(-\alpha)^{1+q-p} x, \quad y^{\prime}=(-\alpha)^{1+q-p} y,
$$

and let

$$
a_{i}^{\prime}=-\alpha a_{i}, \quad i=1, \ldots, p, \quad b_{j}^{\prime}=-\alpha b_{j}, \quad j=1, \ldots, q .
$$

Then, we have that

$$
{ }_{p} S F_{q}\left(a_{1}, \ldots, a_{p} ; b_{1}, \ldots, b_{q} ; \alpha ; x, y\right)={ }_{p} S F_{q}\left(a_{1}^{\prime}, \ldots, a_{p}^{\prime} ; b_{1}^{\prime}, \ldots, b_{q}^{\prime} ; \alpha^{\prime} ; y^{\prime}, x^{\prime}\right) .
$$

Proof. Applying Proposition 6.1 to the definition of ${ }_{p} S F_{q}$ yields

$$
{ }_{p} S F_{q}\left(a_{1}, \ldots, a_{p} ; b_{1}, \ldots, b_{q} ; \alpha ; x, y\right)=\sum_{\lambda} \frac{(-1)^{|\lambda|} b_{\lambda^{\prime}}\left(\alpha^{\prime}\right)}{h_{\lambda}(\alpha)} \frac{\left[a_{1}\right]_{\lambda}^{(\alpha)} \cdots\left[a_{p}\right]_{\lambda}^{(\alpha)}}{\left[b_{1}\right]_{\lambda}^{(\alpha)} \cdots\left[b_{q}\right]_{\lambda}^{(\alpha)}} S P_{\lambda^{\prime}}\left(\alpha^{\prime} ; y, x\right) .
$$

From (4.7) we infer that

$$
b_{\lambda}(\alpha)=\frac{h_{\lambda^{\prime}}\left(\alpha^{\prime}\right)}{\alpha^{|\lambda|} h_{\lambda}(\alpha)}=\frac{1}{b_{\lambda^{\prime}}\left(\alpha^{\prime}\right)} .
$$

Moreover, it is easily verified that

$$
[x]_{\lambda}^{(\alpha)}=(-\alpha)^{-|\lambda|}[-\alpha x]_{\lambda^{\prime}}^{\left(\alpha^{\prime}\right)} .
$$

By applying the last two equations to the series above, we find that

$$
\begin{aligned}
{ }_{p} S F_{q} & \left(a_{1}, \ldots, a_{p} ; b_{1}, \ldots, b_{q} ; \alpha ; x, y\right) \\
& =\sum_{\lambda} \frac{(-\alpha)^{(1+q-p)|\lambda|}}{h_{\lambda^{\prime}}\left(\alpha^{\prime}\right)} \frac{\left[-\alpha a_{1}\right]_{\lambda}^{\left(\alpha^{\prime}\right)} \cdots\left[-\alpha a_{p}\right]_{\lambda}^{\left(\alpha^{\prime}\right)}}{\left[-\alpha b_{1}\right]_{\lambda}^{\left(\alpha^{\prime}\right)} \cdots\left[-\alpha b_{q}\right]_{\lambda}^{\left(\alpha^{\prime}\right)}} S P_{\lambda^{\prime}}\left(\alpha^{\prime} ; y, x\right),
\end{aligned}
$$

which is clearly equivalent to the statement. 


\subsection{Deformed CMS operators}

We proceed to show that all CMS operators of the form (2.18) admit a restriction onto the quotient ring $\Lambda_{\mathbb{F}} / I_{n, m} \simeq \Lambda_{\mathbb{F}, n, m}$, and that these restrictions are given by deformed CMS operators

$$
\mathcal{L}_{n, m}=\sum_{k=0}^{\infty} a_{k} D_{n, m}^{k}+\sum_{\ell=0}^{\infty} b_{\ell} E_{n, m}^{\ell}
$$

with only finitely many coefficients $a_{k}, b_{\ell} \in \mathbb{F}$ non-zero, and where

$$
E_{n, m}^{\ell}=\sum_{i=1}^{n} x_{i}^{\ell} \frac{\partial}{\partial x_{i}}+\sum_{I=1}^{m} y_{I}^{\ell} \frac{\partial}{\partial y_{I}}
$$

and

$$
\begin{aligned}
D_{n, m}^{k}= & \sum_{i=1}^{n} x_{i}^{k} \frac{\partial^{2}}{\partial x_{i}^{2}}+\frac{2}{\alpha} \sum_{i \neq j} \frac{x_{i}^{k}}{x_{i}-x_{j}} \frac{\partial}{\partial x_{i}}-\frac{1}{\alpha} \sum_{I=1}^{m} y_{I}^{k} \frac{\partial^{2}}{\partial y_{I}^{2}}-2 \sum_{I \neq J} \frac{y_{I}^{k}}{y_{I}-y_{J}} \frac{\partial}{\partial y_{I}} \\
& -2 \sum_{i, I} \frac{1}{x_{i}-y_{I}}\left(x_{i}^{k} \frac{\partial}{\partial x_{i}}+\frac{1}{\alpha} y_{I}^{k} \frac{\partial}{\partial y_{I}}\right)-k\left(1+\frac{1}{\alpha}\right) \sum_{I=1}^{m} y_{I}^{k-1} \frac{\partial}{\partial y_{I}}
\end{aligned}
$$

Clearly, it is sufficient to consider the differential operators $E^{\ell}$ and $D^{k}$; c.f. Lemma 2.4 and Proposition 2.5.

Proposition 6.3. Let $p_{0}=n-\alpha m$ for some $n, m \in \mathbb{N}_{0}$. Then, for all $\ell, k \in \mathbb{N}_{0}$, the differential operators $E_{n, m}^{\ell}$ and $D_{n, m}^{k}$ preserve the algebra $\Lambda_{\mathbb{F}, n, m}$. Moreover, they are the unique operators on $\Lambda_{\mathbb{F}, n, m}$ such that the diagrams

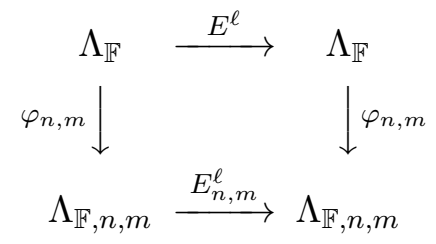

and

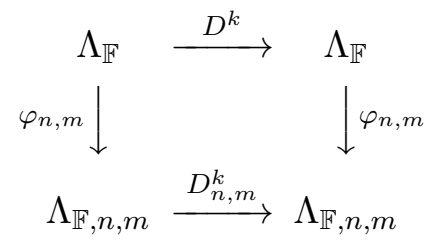

are commutative.

Proof. In order to simplify the proof somewhat it is convenient to collect $x$ and $y$ into a sequence $z=\left(z_{1}, \ldots, z_{n+m}\right)$ by setting

$$
z_{i}= \begin{cases}x_{i}, & 1 \leq i \leq n, \\ y_{i-n}, & n+1 \leq i \leq m .\end{cases}
$$

Also, we introduce a map $\rho:\{1, \ldots, n+m\} \rightarrow \mathbb{F}$ by specifying the value of $\rho(i)$ according to

$$
\rho(i)= \begin{cases}1, & 1 \leq i \leq n, \\ -\alpha, & n+1 \leq i \leq m .\end{cases}
$$


It is clear that

$$
p_{r, \alpha}(x, y)=\sum_{i=1}^{n+m} \rho(i) z_{i}
$$

Furthermore, it is readily verified that $E_{n, m}^{\ell}$ and $D_{n, m}^{k}$ are given by

$$
E_{n, m}^{\ell}=\sum_{i=1}^{n+m} z_{i}^{\ell-1} \frac{\partial}{\partial z_{i}}
$$

and

$$
D_{n, m}^{k}=\sum_{i=1}^{n+m} \frac{1}{\rho(i)} z_{i}^{k} \frac{\partial^{2}}{\partial z_{i}^{2}}+\frac{2}{\alpha} \sum_{i \neq j} \rho(j) \frac{z_{i}^{k}}{z_{i}-z_{j}} \frac{\partial}{\partial z_{i}}-k \sum_{i=1}^{n+m}\left(1-\frac{1}{\rho(i)}\right) z_{i}^{k-1} \frac{\partial}{\partial z_{i}}
$$

respectively.

Proceeding in analogy with the proof of Lemma 2.4, we observe that

$$
\varphi_{n, m}\left(E^{\ell} p_{r}\right)=r p_{r, \alpha}(x, y)=E_{n, m}^{\ell}\left(\varphi_{n, m} p_{r}\right)
$$

if we employ the convention $p_{0, \alpha}(x, y) \equiv n-\alpha m$. This yields (6.6a). Turning now to $D_{n, m}^{k}$, we note that

$$
D_{n, m}^{k} p_{r, \alpha}(z) p_{s, \alpha}(z)=2 r s p_{r+s+k-2, \alpha}(z)+p_{r, \alpha}(z)\left(D_{n, m}^{k} p_{s, \alpha}(z)\right)+p_{s, \alpha}(z)\left(D_{n, m}^{k} p_{r, \alpha}(z)\right),
$$

and deduce by direct computations that

$$
\begin{aligned}
2 \sum_{i \neq j} \rho(j) \frac{z_{i}^{k}}{z_{i}-z_{j}} \frac{\partial}{\partial z_{i}} p_{r, \alpha}(z) & =r \sum_{i \neq j} \sum_{m=0}^{r+k-2} \rho(i) \rho(j) z_{i}^{r+k-2} z_{j}^{m} \\
& =r\left(\sum_{m=0}^{r+k-2} p_{r+k-2-m, \alpha}(z) p_{m, \alpha}(z)-\sum_{i=1}^{n+m} \rho(i)^{2} z_{i}^{r+k-2}\right) .
\end{aligned}
$$

Using the fact that

$$
r(r-1)-r(r+k-1) \frac{\rho(i)^{2}}{\alpha}+r k(1-\rho(i))=r(r-1) \rho(i)-\frac{1}{\alpha} r(r+k-1) \rho(i),
$$

it is now straightforward to deduce that

$$
\begin{aligned}
& D_{n, m}^{k} p_{r, \alpha}(z) p_{s, \alpha}(z)=r(r-1) p_{r+k-2, \alpha}(z) p_{s, \alpha}(z)+2 r s p_{r+q+k-2, \alpha}(z) \\
& \quad+s(s-s) p_{r, \alpha}(z) p_{s+k-2, \alpha}(z)+\frac{r}{\alpha} \sum_{m=0}^{r+k-2}\left(p_{r+k-2-m, \alpha}(z) p_{m, \alpha}(z)-p_{r+k-2, \alpha}(z)\right) .
\end{aligned}
$$

Comparing this result with Definition 2.3 we obtain $(6.6 \mathrm{~b})$.

Finally, uniqueness is clear since $\varphi_{n, m}$ is surjective.

The following lemma will be useful in establishing duality relations for the super Hermite and Laguerre polynomials:

Lemma 6.4. We have that

$$
D_{n, m}^{k}(\alpha)=-\frac{1}{\alpha}\left(D_{m, n}^{k}(1 / \alpha)+k(1+\alpha) E_{m, n}^{k-1}\right) .
$$


Proof. We first define

$$
\Delta_{n, m}(\alpha)=\sum_{i, I} \frac{1}{x_{i}-y_{I}}\left(x_{i}^{k} \frac{\partial}{\partial x_{i}}+\frac{1}{\alpha} y_{I}^{k} \frac{\partial}{\partial y_{I}}\right) .
$$

Then, we can decompose $D_{n, m}^{k}$ as follows:

$$
D_{n, m}^{k}(\alpha)=D_{n, x}^{k}(\alpha)-\frac{1}{\alpha} D_{m, y}^{k}(1 / \alpha)-2 \Delta_{n, m}(\alpha)-k\left(1+\frac{1}{\alpha}\right) E_{m, y}^{k-1},
$$

where the subscript $x$ indicates that the operator in questions acts in the indeterminates $x$, and similarly for the subscript $y$. We observe that $\Delta_{n, m}(\alpha)=-(1 / \alpha) \Delta_{m, n}(1 / \alpha)$. Consequently, we can rewrite the operator $D_{n, m}^{k}(\alpha)$ in the form

$$
-\frac{1}{\alpha}\left(D_{m, x}^{k}(1 / \alpha)-\alpha D_{n, y}^{k}(\alpha)-2 \Delta_{m, n}(1 / \alpha)+k(1+\alpha) E_{m, y}^{k-1}\right) .
$$

The desired formula now follows from the fact that $E_{m, n}^{\ell}=E_{n, m}^{\ell}$ and the fact that $E_{m, y}^{k-1}=$ $E_{n, m}^{k-1}-E_{n, x}^{k-1}$.

\subsection{Super Hermite polynomials}

As before, we assume that $p_{0}=n-\alpha m$ for some $n, m \in \mathbb{N}_{0}$. It directly follows from Proposition 6.3 that

$$
\varphi_{n, m} \circ \mathcal{L}^{H}=\mathcal{L}_{n, m}^{H} \circ \varphi_{n, m}
$$

with $\mathcal{L}^{H}$ as in (4.1) (for $\nu=1$ ) and $\mathcal{L}_{n, m}^{H}$ obtained by substituting $D_{n, m}^{k}$ for $D^{k}$ and $E_{n, m}^{\ell}$ for $E^{\ell}{ }^{2}$ This observation suggests the following definition:

Definition 6.5. Let $\lambda$ be a partition such that $(n+1, m+1) \notin \lambda$. We then define the super Hermite polynomial $S H_{\lambda}(\alpha ; x, y)$ by

$$
S H_{\lambda}(x, y)=\varphi_{n, m}\left(H_{\lambda}\right) \text {. }
$$

Clearly, the super Hermite polynomials satisfy the eigenvalue equation

$$
\mathcal{L}_{n, m}^{H} S H_{\lambda}(x, y)=-2|\lambda| S H_{\lambda}(x, y) .
$$

We note that the super Hermite polynomials coincide with the (reduced) eigenfunctions constructed for Case I in Section 4 of [12]. This follows from the fact that, for fixed $\lambda$, the eigenvalue equation above has a unique solution with a triangular expansion in super Jack polynomials; c.f. Theorem 4 in Appendix B and the discussion in Section 5.3 of [12].

Using the results obtained in Section 4 we can also produce higher-order eigenoperators. For $f \in \Lambda_{\mathbb{F}, \alpha}$, we let $\mathcal{L}_{f, n, m}^{H}$ denote the deformed CMS operator obtained from (4.16) by substituting $\mathcal{L}_{f, n, m}$ for $\mathcal{L}_{f}$ and $D_{n, m}^{0}$ for $D^{0}$.

\footnotetext{
${ }^{2}$ The operator $\mathcal{L}_{n, m}^{H}$ corresponds to the operator $-\tilde{H}_{n, m}$ defined in [12] by equation (44) and Case I of Table 2 together with $\alpha=1 / \kappa, \nu^{2}=\omega, x_{i}=z_{i}$, and $y_{i}=\tilde{z}_{i} \cdot \mathcal{L}_{n, m}^{H}$ can also be obtained from the operator in Theorem 9 in [11] given by equation (15) by first performing a similarity transformation by the function $\exp \left(-\omega \sum_{i=1}^{n+m} y_{i}^{2}\right)$, then setting $y_{i}=x_{i}, i=1, \ldots, n, y_{n+i}=y_{i} / \sqrt{k}, i=1, \ldots, m$, and finally $k=-1 / \alpha$ and $\omega=\nu^{2}$.
} 
Proposition 6.6. Let $p_{0}=n-\alpha m$ for some $n, m \in \mathbb{N}_{0}$, and let $f \in \Lambda_{\mathbb{F}, \alpha}$. Then, the deformed $C M S$ operator $\mathcal{L}_{f, n, m}^{H}$ is the unique operator on $\Lambda_{\mathbb{F}, n, m}$ such that the diagram

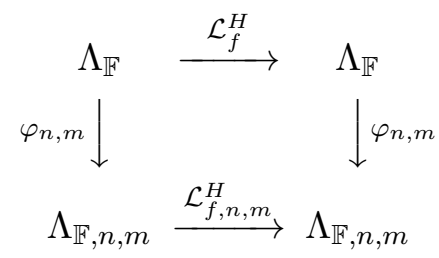

is commutative. Moreover, we have that

$$
\mathcal{L}_{f, n, m}^{H} S H_{\lambda}(x, y)=f(\lambda) S H_{\lambda}(x, y) .
$$

Proof. The commutativity of the diagram, as well as uniqueness, follows immediately from (6.2), Proposition 6.3, and the definition of $\mathcal{L}_{f, n, m}^{H}$. The fact that the super Hermite polynomials are eigenfunctions of this operator, with the stated eigenvalues, is then a direct consequence of Corollary 4.8 .

An immediate consequence of the previous proposition is the integrability of the deformed CMS model in the Hermite case (also called rational CMS model with harmonic confinement). In the special case $n=1$ or $m=1$, the integrability of this model was already known [6, Theorem 3], and for $1 / \alpha \in \mathbb{Z}$, but general values of $n$ and $m$, a different proof can be found in [11].

It also follows from Proposition 6.6 that the kernel $\operatorname{ker}\left(\varphi_{n, m}\right)$ of $\varphi_{n, m}$ is invariant under all differential operators $\mathcal{L}_{f}^{H}, f \in \Lambda_{\mathbb{F}, \alpha}$. The uniqueness result in Theorem 4.22 implies that

$$
\operatorname{ker}\left(\varphi_{n, m}\right)=\mathbb{F}\left\langle H_{\lambda}:(n+1, m+1) \in \lambda\right\rangle,
$$

and we thus arrive at the first equality in (6.1).

We proceed to deduce a duality relation for the super Hermite polynomials, analogous to that given in Proposition 6.1 for the super Jack polynomials. To this end, we should re-introduce the parameter $\nu^{2}$ via the homomorphism $\sigma_{\nu}$ :

$$
S H_{\lambda}\left(\alpha, \nu^{2} ; x, y\right):=\varphi_{n, m}\left(\sigma_{\nu}\left(H_{\lambda}(\alpha)\right)\right)
$$

c.f., the paragraph containing (4.2). As for the Hermite symmetric functions, this duality relation can be directly inferred from a constructive definition of the super Hermite polynomials. If we apply the homomorphism $\varphi_{n, m}$ to both sides of (4.4), then Proposition 6.3 implies the following result:

Proposition 6.7. Let $\lambda$ be a partition such that $(n+1, m+1) \notin \lambda$, and set $L=\lfloor|\lambda| / 2\rfloor$. Then, we have that

$$
S H_{\lambda}(x, y)=\exp _{L}\left(-\frac{1}{4 \nu^{2}} D_{n, m}^{0}\right) S P_{\lambda}(x, y) .
$$

Since $-\alpha D_{n, m}^{0}(\alpha)=D_{m, n}^{0}(1 / \alpha)$ (c.f., Lemma 6.4$)$, this immediately yields the desired duality relation for the super Hermite polynomials.

Proposition 6.8. The super Hermite polynomials satisfy the duality relation

$$
S H_{\lambda}\left(\alpha, \nu^{2} ; x, y\right)=(-1)^{|\lambda|} b_{\lambda^{\prime}}^{(1 / \alpha)} S H_{\lambda^{\prime}}\left(1 / \alpha,-\alpha \nu^{2} ; y, x\right) .
$$


By applying the homomorphism $\varphi_{n, m}$, we can restrict further results from Section 4 to the super Hermite polynomials. In particular, in this way we obtain the generating function expansion

$$
\sum_{\lambda} \frac{1}{h_{\lambda} \epsilon_{n-\alpha m}\left(P_{\lambda}\right)} S H_{\lambda}(x, y) \otimes S P_{\lambda}(z, w)={ }_{0} \mathscr{S} \mathscr{F}_{0}(x, y ; z, w) e^{-\frac{1}{4} p_{2, \alpha}(z, w)}
$$

with

$$
{ }_{0} \mathscr{S} \mathscr{F}_{0}(x, y ; z, w):=\sum_{\lambda} \frac{1}{h_{\lambda} \epsilon_{n-\alpha m}\left(P_{\lambda}\right)} S P_{\lambda}(x, y) S P_{\lambda}(z, w),
$$

where, in both formulae, the sums extend over all partitions $\lambda$ such that $(n+1, m+1) \notin \lambda$. We leave the straightforward deduction of further such properties of the super Hermite polynomials to the interested reader.

\subsection{Super Laguerre polynomials}

The theory of super Laguerre polynomials can be developed in complete analogy with that of the super Hermite polynomials. First of all, we have that

$$
\varphi_{n, m} \circ \mathcal{L}^{L}=\mathcal{L}_{n, m}^{L} \circ \varphi_{n, m}
$$

with $\mathcal{L}^{L}$ as in (5.1) (for $\nu=1$ ) and $\mathcal{L}_{n, m}^{L}$ obtained by substituting $D_{n, m}^{k}$ for $D^{k}$ and $E_{n, m}^{\ell}$ for $E^{\ell}{ }^{3}$ This fact suggests the following definition:

Definition 6.9. Let $\lambda$ be a partition such that $(n+1, m+1) \notin \lambda$. We then define the super Laguerre polynomial $S L_{\lambda}(\alpha, a ; x, y)$ by

$$
S L_{\lambda}(x, y)=\varphi_{n, m}\left(L_{\lambda}\right) .
$$

In a similar manner to the Hermite case one can verify that the super Laguerre polynomials coincide with the (reduced) eigenfunctions constructed for Case IV in Section 4 of [12].

Higher-order eigenoperators can be constructed using Proposition 5.6.

Proposition 6.10. Let $p_{0}=n-\alpha m$ for some $n, m \in \mathbb{N}_{0}$, and fix $f \in \Lambda_{\mathbb{E}, \alpha}$. Then, the deformed $C M S$ operator $\mathcal{L}_{f, n, m}^{L}$, obtained from (5.4) by substituting $\mathcal{L}_{f, n, m}$ for $\mathcal{L}_{f}, D_{n, m}^{1}$ for $D^{1}$ and $E_{n, m}^{0}$ for $E^{0}$, is the unique operator on $\Lambda_{\mathbb{F}, n, m}$ such that the diagram

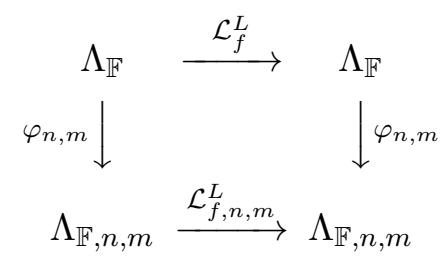

is commutative. Moreover, we have that

$$
\mathcal{L}_{f, n, m}^{L} S L_{\lambda}(x, y)=f(\lambda) S L_{\lambda}(x, y) .
$$

\footnotetext{
${ }^{3}$ In [12], take the operator $-\tilde{H}_{n, m} / 4$ given in equation (44), then choose the Case IV of Table 2 , set $z_{i}=x_{i}$, $\tilde{z}_{i}=y_{i}, \omega=\nu$, and $\kappa=1 / \alpha$. This gives the operator $\mathcal{L}_{n, m}^{L}$ defined above. One can also obtain the same operator from the operator in Theorem 9 in [11] given by equation (18) in a similar manner to the Hermite case after first performing a change of variables $y_{i} \rightarrow \sqrt{y_{i}}$.
} 
We note that integrability of the deformed CMS model in Laguerre case (i.e., type B), which now directly follows from the above proposition, had been previously proved for the case with $n=m=1$ [6, Theorem 5], and for $1 / \alpha \in \mathbb{Z}$, but general values of $n$ and $m$, in [11].

Reintroducing the parameter $\nu$, we can easily establish a duality relation using the following constructive definition:

Proposition 6.11. Let $\lambda$ be a partition and set $L=|\lambda|$. Then, we have that

$$
S L_{\lambda}=\exp _{L}\left(-\frac{1}{\nu}\left(D_{n, m}^{1}+(a+1) E_{n, m}^{0}\right)\right) S P_{\lambda} .
$$

A duality relation for the super Laguerre polynomials is now straightforward to infer.

Proposition 6.12. We have that

$$
S L_{\lambda}(\alpha, a, \nu ; x, y)=(-1)^{|\lambda|} b_{\lambda^{\prime}}^{(1 / \alpha)} S L_{\lambda^{\prime}}(1 / \alpha,-\alpha a,-\alpha \nu ; y, x) .
$$

Further results can be obtained by applying $\varphi_{n, m}$ to results obtained in Section 5 .

\section{A Differential operators on the symmetric functions}

To establish a wider context for our results, we proceed to consider the notion of differential operators on the ring of symmetric functions. In particular, this will make it clear that the infinite-dimensional CMS operators we have considered indeed are differential operators on this ring.

Throughout this section, $\mathbb{F}$ will denote an arbitrary, but fixed, field of characteristic zero. Correspondingly, we have $\Lambda_{\mathbb{F}} \equiv \mathbb{F} \otimes_{\mathbb{Z}} \Lambda$.

For a module over a commutative algebra, there is a natural notion of differential operators on said module; see, e.g., Chapter 2 of Heyneman and Sweedler [15]. In the present case of $\Lambda_{\mathbb{F}}$, this general notion results in the following definition: we first set $\mathscr{D}_{-1}\left(\Lambda_{\mathbb{F}}\right)=\{0\}$, and then let

$$
\mathscr{D}_{k}\left(\Lambda_{\mathbb{F}}\right)=\left\{u \in \operatorname{End}\left(\Lambda_{\mathbb{F}}\right): u \circ p-p \circ u \in \mathscr{D}_{k-1}\left(\Lambda_{\mathbb{F}}\right) \forall p \in \Lambda_{\mathbb{F}}\right\}
$$

for $k \geq 0$. Following standard terminology, we shall refer to the elements in the space $\mathscr{D}_{k}\left(\Lambda_{\mathbb{F}}\right) \backslash$ $\mathscr{D}_{k-1}\left(\Lambda_{\mathbb{F}}\right)$ as the (linear and homogeneous) differential operators on $\Lambda_{\mathbb{F}}$ of order $k$. Moreover, we shall write $\mathscr{D}\left(\Lambda_{\mathbb{F}}\right)$ for the space of all such differential operators, i.e., $\mathscr{D}\left(\Lambda_{\mathbb{F}}\right)=\cup_{k \geq-1} \mathscr{D}_{k}\left(\Lambda_{\mathbb{F}}\right)$.

We record the following two elementary facts: $\mathscr{D}_{0}\left(\Lambda_{\mathbb{F}}\right)=\Lambda_{\mathbb{F}}$ (if we identify any $p \in \Lambda_{\mathbb{F}}$ with the corresponding operator $q \mapsto p q)$, for any $u \in \mathscr{D}_{k}\left(\Lambda_{\mathbb{F}}\right)$ and $v \in \mathscr{D}_{k^{\prime}}\left(\Lambda_{\mathbb{F}}\right)$, the composition $u \circ v \in \mathscr{D}_{k+k^{\prime}}\left(\Lambda_{\mathbb{F}}\right)$. Both of these facts are easy to verify directly, and can also be inferred from the general theory mentioned above.

For our purposes, we require a more explicit realisation of $\mathscr{D}\left(\Lambda_{\mathbb{F}}\right)$, given by a set of generators. We thus note that, since the power sums $p_{r}$ freely generate $\Lambda_{\mathbb{F}}$, we can, for each $r \in \mathbb{N}$, define $\partial\left(p_{r}\right) \in \mathscr{D}_{1}\left(\Lambda_{\mathbb{F}}\right)$ by $\partial\left(p_{r}\right) 1=0$ and

$$
\partial\left(p_{r}\right) p_{s}= \begin{cases}1, & r=s \\ 0, & r \neq s\end{cases}
$$

The assignment $p_{r} \mapsto \partial\left(p_{r}\right)$ extends uniquely to an $\mathbb{F}$-algebra homomorphism $\partial: \Lambda_{\mathbb{F}} \rightarrow \mathscr{D}\left(\Lambda_{\mathbb{F}}\right)$, given by

$$
\partial\left(\sum_{\lambda} a_{\lambda} p_{\lambda}\right)=\sum_{\lambda} a_{\lambda} \partial\left(p_{\lambda_{1}}\right) \partial\left(p_{\lambda_{2}}\right) \cdots .
$$


More generally, we can consider the subring of $\mathscr{D}\left(\Lambda_{\mathbb{F}}\right)$ generated by the differential operators $\partial\left(p_{r}\right)$ over $\Lambda_{\mathbb{F}}$ :

$$
A:=\mathbb{F}\left[p_{1}, p_{2}, \ldots, \partial\left(p_{1}\right), \partial\left(p_{2}\right), \ldots\right] \subset \mathscr{D}\left(\Lambda_{\mathbb{F}}\right) .
$$

In the case of the finitely generated algebra $\Lambda_{\mathbb{F}, n}$ the corresponding subring would coincide with $\mathscr{D}\left(\Lambda_{\mathbb{F}, n}\right)$. However, this is not the case for $A$ and $\mathscr{D}\left(\Lambda_{\mathbb{F}}\right)$. In fact, none of the differential operators we focus on in this paper are contained in $A$. For example, it is easy to verify that the formal series

$$
E_{1}=\sum_{r=1}^{\infty} p_{r} \partial\left(p_{r}\right)
$$

defines a first-order differential operator on $\Lambda_{\mathbb{F}}$ by

$$
E_{1} q \equiv \sum_{r=1}^{\infty} p_{r} \partial\left(p_{r}\right) q, \quad q \in \Lambda_{\mathbb{F}}
$$

Indeed, since any $q \in \Lambda_{\mathbb{F}}$ can be written uniquely as a finite linear combination of terms of the form $p_{\lambda}=p_{\lambda_{1}} p_{\lambda_{2}} \cdots$, the infinite sum in (A.2) contains only a finite number of non-zero terms. Hence, $E_{1} q \in \Lambda_{\mathbb{F}}$, and the fact that $E_{1} \in \mathscr{D}_{1}\left(\Lambda_{\mathbb{F}}\right)$ is now clear from the definition of $\mathscr{D}_{1}\left(\Lambda_{\mathbb{F}}\right)$.

We proceed to enlarge the ring $A$ such that we obtain all of $\mathscr{D}\left(\Lambda_{\mathbb{F}}\right)$. The above example indicates that we should include also formal power series in the differential operators $\partial\left(p_{r}\right)$ with coefficients in $\Lambda_{\mathbb{F}}$. However, we should ensure that we do not include formal series of infinite order. To illustrate this point, let us consider

$$
D=\sum_{k=1}^{\infty} \partial\left(p_{1}\right)^{k}
$$

Interchanging the order of summation and differentiation, as in (A.2), this formal series does define an element in $\operatorname{End}\left(\Lambda_{\mathbb{F}}\right)$. But, suppose that $D \in \mathscr{D}_{l}\left(\Lambda_{\mathbb{F}}\right)$ for some $l \in \mathbb{N}_{0}$. By definition, this means that

$$
\left[\cdots\left[\left[D, q_{1}\right], q_{2}\right], \cdots, q_{l+1}\right]=0
$$

for any $q_{1}, \ldots, q_{l+1} \in \Lambda_{\mathbb{F}}$. However, if we set $q_{1}=\cdots=q_{l+1}=p_{1}$, then we obtain

$$
\sum_{k=0}^{\infty} \frac{(l+k) !}{k !} \partial\left(p_{1}\right)^{k}
$$

which is clearly non-zero. Hence, $D \notin \mathscr{D}\left(\Lambda_{\mathbb{F}}\right)$.

In order to make these remarks precise, we note that $A$ inherits a natural filtration from $\mathscr{D}\left(\Lambda_{\mathbb{F}}\right)$, given by the order of the differential operators:

$$
A^{0} \subset A^{1} \subset \cdots \subset A^{k} \subset \cdots
$$

with

$$
A^{k}=A \cap \mathscr{D}_{k}\left(\Lambda_{\mathbb{F}}\right)=\left\{\sum_{\ell(\lambda) \leq k} q_{\lambda} \partial\left(p_{\lambda}\right): q_{\lambda} \in \Lambda_{\mathbb{F}}\right\} .
$$


Indeed, we clearly have $A=\cup_{k \geq 0} A^{k}$ and $A^{k} A^{l} \subset A^{k+l}$ for all $k, l \in \mathbb{N}_{0}$. On each such submodule $A^{k} \subset A$, we can then introduce a filtration

$$
A^{k}=A_{0}^{k} \supset A_{1}^{k} \supset \cdots \supset A_{n}^{k} \supset \cdots,
$$

where

$$
A_{n}^{k}=\left\{\sum_{\substack{\ell(\lambda) \leq k \\|\lambda| \geq n}} q_{\lambda} \partial\left(p_{\lambda}\right): q_{\lambda} \in \Lambda_{\mathbb{F}}\right\} .
$$

By $\hat{A}^{k}$ we denote the corresponding completion of $A^{k}$; see, e.g., Chapter 10 in Atiyah and Macdonald [1] for a general discussion of the process of completion in the context of (commutative) algebra. For the discussion below, it will be important to note that each element in $\hat{A}^{k}$ can be uniquely identified with a formal power series in the first-order differential operators $\partial\left(p_{r}\right)$ of order not greater than $k$, and vice versa. We proceed to show that the filtered module

$$
\hat{A}=\bigcup_{k \geq 0} \hat{A}^{k}
$$

can be naturally identified with $\mathscr{D}\left(\Lambda_{\mathbb{F}}\right)$.

Proposition A.1. Any element $D=\sum_{\lambda} q_{\lambda} \partial\left(p_{\lambda}\right) \in \hat{A}$ defines a differential operator on $\Lambda_{\mathbb{F}}$ by

$$
D p \equiv \sum_{\lambda} q_{\lambda} \partial\left(p_{\lambda}\right) p, \quad p \in \Lambda_{\mathbb{F}} .
$$

In this sense, $\hat{A}^{k} \backslash \hat{A}^{k-1}=\mathscr{D}_{k}\left(\Lambda_{\mathbb{F}}\right) \backslash \mathscr{D}_{k-1}\left(\Lambda_{\mathbb{F}}\right)$ for all $k \in \mathbb{N}_{0}$, and consequently $\hat{A}=\mathscr{D}\left(\Lambda_{\mathbb{F}}\right)$.

Proof. Given $D \in \hat{A}$ as in the statement and $p \in \Lambda_{\mathbb{F}}$, it is clear that $D p$ is a finite linear combination of $q_{\lambda} \in \Lambda_{\mathbb{F}}$. Hence, $D \in \operatorname{End}\left(\Lambda_{\mathbb{F}}\right)$. By induction on $k$ it is now easy to verify that $\hat{A}^{k} \subset \mathscr{D}_{k}\left(\Lambda_{\mathbb{F}}\right)$.

We proceed to establish injectivity, i.e., that given $D, D^{\prime} \in \hat{A}$ we have $\left(D-D^{\prime}\right) p=0$ for all $p \in \Lambda_{\mathbb{F}}$ if and only if $D=D^{\prime}$. The first part of this claim clearly holds true. Suppose therefore that $D \neq D^{\prime}$. This means that

$$
D-D^{\prime}=\sum_{\lambda} q_{\lambda} \partial\left(p_{\lambda}\right)
$$

with at least one coefficient $q_{\lambda} \neq 0$. Among all such non-zero coefficients, fix one with the corresponding partition $\lambda$ having minimal length $\ell(\lambda)$. It follows that

$$
\left(D-D^{\prime}\right) p_{\lambda}=q_{\lambda} \neq 0
$$

which proves the second part of the claim.

In order to establish surjectivity, i.e., that any $D \in \mathscr{D}_{k}\left(\Lambda_{\mathbb{F}}\right) \backslash \mathscr{D}_{k-1}\left(\Lambda_{\mathbb{F}}\right)$ is of the form $D=$ $\sum_{\ell(\lambda)=k} q_{\lambda} \partial\left(p_{\lambda}\right) \in \hat{A}^{k} \backslash \hat{A}^{k-1}$. where $A_{-1}:=\{0\}$, we proceed by induction on the order $k$. For $k=0$, this claim is obvious. Suppose that $k>0$. Fix $D \in \mathscr{D}_{k}\left(\Lambda_{\mathbb{F}}\right) \backslash \mathscr{D}_{k-1}\left(\Lambda_{\mathbb{F}}\right)$, and let

$$
f_{r}=\left[D, p_{r}\right], \quad r \in \mathbb{N} .
$$

Then, by the induction assumption, we have that

$$
f_{r} \in \mathscr{D}_{k-1}\left(\Lambda_{\mathbb{F}}\right) \backslash \mathscr{D}_{k-2}\left(\Lambda_{\mathbb{F}}\right)=\hat{A}^{k-1} \backslash \hat{A}^{k-2} .
$$


It follows that

$$
D^{\prime}:=\frac{1}{k} \sum_{r} f_{r} \partial\left(p_{r}\right) \in \hat{A}_{k} \backslash \hat{A}_{k-1}
$$

We note that $\mathscr{D}\left(\Lambda_{\mathbb{F}}\right)$ is a Lie algebra (with Lie bracket given by $[u, v]=u \circ v-v \circ u$ ); c.f., Lemma 2.1.1. in Heyneman and Sweedler [15]. By the Jacobi identity, we thus infer that

$$
\left[f_{r}, p_{s}\right] \equiv\left[\left[D, p_{r}\right], p_{s}\right]=\left[\left[D, p_{s}\right], p_{r}\right] \equiv\left[f_{s}, p_{r}\right], \quad \forall r, s \in \mathbb{N} .
$$

We also note that, for any partition $\lambda$,

$$
\sum_{r}\left[\partial\left(p_{\lambda}\right), p_{r}\right]=\ell(\lambda)
$$

Since each $f_{r}=\sum_{\ell(\lambda)=k-1} q_{\lambda} \partial\left(p_{\lambda}\right)$ for some $q_{\lambda} \in \Lambda_{\mathbb{F}}$, these facts combine to yield

$$
\left[D-D^{\prime}, p_{s}\right]=f_{s}-\frac{1}{k} f_{s}-\frac{1}{k} \sum_{r}\left[f_{s}, p_{r}\right] \partial\left(p_{r}\right)=0
$$

Hence, $D-D^{\prime} \in \mathscr{D}_{0}\left(\Lambda_{\mathbb{F}}\right)=\hat{A}^{0}$, and surjectivity follows.

Finally, we provide a simple criterion for a differential operator in $\mathscr{D}\left(\Lambda_{\mathbf{F}}\right)$ to be continuous

with respect to the topology given by the ideal $U=\oplus_{k \geq 1} \Lambda_{\mathbf{F}}^{k}$; c.f. the paragraph preceding Proposition 3.2. To this end, we introduce a notion of degree, deg : $\mathscr{D}\left(\Lambda_{\mathbb{F}}\right) \rightarrow \mathbb{Z} \cup\{-\infty\}$, by requiring that

$$
\operatorname{deg}(D) \geq m \quad \text { if } \quad D U^{n} \subset U^{n+m} \quad \forall n \in \mathbb{N}_{0},
$$

where we make the identification $U^{n+m} \equiv U^{0}$ for $m+n<0$, and setting $\operatorname{deg}(D)=-\infty$ if no such integer $m$ exists. It is clear that we have the following lemma:

Lemma A.2. Let $D \in \mathscr{D}\left(\Lambda_{\mathbb{F}}\right)$. If $\operatorname{deg}(D)>-\infty$, then $D$ is continuous with respect to the $U$-adic topology.

As a simple example of a differential operator $D \in \mathscr{D}\left(\Lambda_{\mathbb{F}}\right)$ with $\operatorname{deg}(D)=-\infty$, we note

$$
D=\sum_{r=1}^{\infty} \partial\left(p_{r}\right) .
$$

Indeed, this follows directly from the fact that $D p_{r}=1$ for all $r \in \mathbb{N}_{0}$.

\section{B CMS operators on the symmetric functions}

In this appendix we record a few technical details on the differential operators $E^{\ell}$ and $D^{k}$ that are used throughout the paper. In addition, we shall isolate certain results that hold true not only in the Hermite and Laguerre cases, including the fact that a generic infinite-dimensional CMS operator of second order has a complete set of eigenfunctions in $\Lambda_{\mathbf{F}}$.

As in the case of a finite number of variables, these operators are not algebraically independent of each other. By direct computations, we deduce the following relations: 
Lemma B.1. For $\ell, k \in \mathbb{N}_{0}$,

$$
\begin{aligned}
& {\left[E^{k}, E^{\ell+1}\right]=(\ell+1) E^{k+\ell},} \\
& {\left[E^{0}, D^{k+1}\right]=(k+1) D^{k},} \\
& {\left[E^{1}, D^{k}\right]=(k-2) D^{k},} \\
& {\left[E^{2}, D^{k}\right]=(k-4) D^{k+1}+2\left(\frac{p_{0}-1}{\alpha}-1\right) E^{k},} \\
& {\left[E^{k}, p_{\ell+1}\right]=(\ell+1) p_{k+\ell},} \\
& {\left[D^{k}, p_{\ell+1}\right]=2(\ell+1) E^{k+\ell}+\ell(\ell+1) p_{k+\ell-1}+\frac{\ell+1}{\alpha} \sum_{m=0}^{k+\ell-1}\left(p_{k+\ell-m-1} p_{m}-p_{k+\ell-1}\right) .}
\end{aligned}
$$

When constructing eigenfunctions of the CMS operators

$$
\mathcal{L}=\sum_{k=0}^{2} a_{k} D^{k}+\sum_{\ell=0}^{1} b_{\ell} E^{\ell}, \quad a_{k}, b_{\ell} \in \mathbf{F},
$$

it is important to know their action on Jack's symmetric functions. (We could of course use another linear basis for $\Lambda_{\mathbf{F}}$, but the action on Jack's symmetric functions is particularly simple.) Clearly, it is sufficient to consider the operators $E^{\ell}$ and $D^{k}$.

To this end, we recall that Lassalle [27] defined generalised binomial coefficients $\left(\begin{array}{l}\lambda \\ \mu\end{array}\right)$ by the series expansion

$$
\frac{P_{\lambda}\left(x_{1}+1, \ldots, x_{n}+1\right)}{P_{\lambda}\left(1^{n}\right)}=\sum_{\mu \subseteq \lambda}\left(\begin{array}{l}
\lambda \\
\mu
\end{array}\right) \frac{P_{\mu}\left(x_{1}, \ldots, x_{n}\right)}{P_{\mu}\left(1^{n}\right)} .
$$

It was later shown that these binomial coefficients are independent of $n$; see, e.g., [32]. As a consequence, we can deduce the following generalisation to Jack's symmetric functions:

Proposition B.2. Let $t_{\gamma}$ be the translation homomorphism defined in (4.20). For any partition $\lambda$, we have

$$
\frac{t_{1}\left(P_{\lambda}\right)}{\epsilon_{p_{0}}\left(P_{\lambda}\right)}=\sum_{\mu \subseteq \lambda}\left(\begin{array}{l}
\lambda \\
\mu
\end{array}\right) \frac{P_{\mu}}{\epsilon_{p_{0}}\left(P_{\mu}\right)}
$$

Proof. We fix the partition $\lambda$, and let $k=|\lambda|$. Then, we expand the difference between the left- and right-hand side of (B.4) in terms of Jack's symmetric functions,

$$
\frac{t_{1}\left(P_{\lambda}\right)}{\epsilon_{p_{0}}\left(P_{\lambda}\right)}-\sum_{\mu \subseteq \lambda}\left(\begin{array}{l}
\lambda \\
\mu
\end{array}\right) \frac{P_{\mu}}{\epsilon_{p_{0}}\left(P_{\mu}\right)}=\sum_{|\mu| \leq|\lambda|} a_{\lambda \mu}\left(p_{0}\right) P_{\mu} .
$$

By applying the restriction homomorphism $\rho_{n}$ we infer from (B.3) that $a_{\lambda \mu}(n)=0$ as long as $n \geq k$. (If $n<k$, then $P_{\mu}\left(x_{1}, \ldots, x_{n}\right)$ migh be zero.) Moreover, it is clear from Stanley's specialisation formula (2.10) and the definition of $t_{1}$ that $a_{\lambda \mu}\left(p_{0}\right)$ is a rational function of $p_{0}$. Since it vanishes at infinitely many distinct points, we can thus conclude that $a_{\lambda \mu} \equiv 0$.

Similarly, starting from the formula in Section 3 of Lassalle [27], we can deduce an expression for the lowest degree Pieri formula for Jack's symmetric functions given in terms of binomial coefficients. 
Proposition B.3. For any partition $\lambda$, we have

$$
p_{1} \frac{P_{\lambda}}{h_{\lambda}}=\sum_{i}\left(\begin{array}{c}
\lambda^{(i)} \\
\lambda
\end{array}\right) \frac{P_{\lambda^{(i)}}}{h_{\lambda^{(i)}}}
$$

where the sum extends over all positive integers $i$ such that $\lambda^{(i)}$ is a partition.

Moreover, the coefficients in this formula are known explicitly: $h_{\lambda}$ is given by (2.1) and

$$
\left(\begin{array}{c}
\lambda^{(i)} \\
\lambda
\end{array}\right)=\left(\lambda_{i}+1+\frac{\ell\left(\lambda^{(i)}\right)-i}{\alpha}\right) \prod_{j \neq i} \frac{\alpha\left(\lambda_{i}+1-\lambda_{j}\right)+j-i-1}{\alpha\left(\lambda_{i}+1-\lambda_{j}\right)+j-i} ;
$$

see Section 14 in Lassalle [23] for the latter fact.

Using the two Propositions above it is now straightforward to compute the action of the operators $E^{\ell}$ and $D^{k}$ on Jack's symmetric functions. In particular, we have the following:

Lemma B.4. For any partition $\lambda$,

$$
\begin{aligned}
& E^{2} \frac{P_{\lambda}}{h_{\lambda}}=\sum_{i}\left(\begin{array}{c}
\lambda^{(i)} \\
\lambda
\end{array}\right)\left(\lambda_{i}-\frac{i-1}{\alpha}\right) \frac{P_{\lambda^{(i)}}}{h_{\lambda^{(i)}}}, \\
& E^{1} P_{\lambda}=|\lambda| P_{\lambda} \text {, } \\
& E^{0} \frac{P_{\lambda}}{\epsilon_{p_{0}}\left(P_{\lambda}\right)}=\sum_{i}\left(\begin{array}{c}
\lambda \\
\lambda_{(i)}
\end{array}\right) \frac{P_{\lambda_{(i)}}}{\epsilon_{p_{0}}\left(P_{\lambda_{(i)}}\right)} \\
& D^{2} P_{\lambda}=d_{\lambda} P_{\lambda}, \quad d_{\lambda}=\sum_{i} \lambda_{i}\left(\lambda_{i}-1+\frac{2}{\alpha}\left(p_{0}-i\right)\right), \\
& D^{1} \frac{P_{\lambda}}{\epsilon_{p_{0}}\left(P_{\lambda}\right)}=\sum_{i}\left(\begin{array}{c}
\lambda \\
\lambda_{(i)}
\end{array}\right)\left(\lambda_{i}-1+\frac{p_{0}-i}{\alpha}\right) \frac{P_{\lambda_{(i)}}}{\epsilon_{p_{0}}\left(P_{\lambda_{(i)}}\right)}, \\
& D^{0} \frac{P_{\lambda}}{\epsilon_{p_{0}}\left(P_{\lambda}\right)}=\sum_{i, j}\left(\begin{array}{c}
\lambda \\
\lambda_{(i)}
\end{array}\right)\left(\begin{array}{c}
\lambda_{(i)} \\
\lambda_{(i, j)}
\end{array}\right)\left(\lambda_{i}-\lambda_{j}+\frac{j-i}{\alpha}+\delta_{i j}\right) \frac{P_{\lambda_{(i, j)}}}{\epsilon_{p_{0}}\left(P_{\lambda_{(i, j)}}\right)} .
\end{aligned}
$$

Proof. We shall verify the equations in the order in which they are listed. According to (B.1f),

$$
E^{2}=\frac{1}{2}\left[D^{2}, p_{1}\right]-\frac{1}{\alpha}\left(p_{0}-1\right) p_{1} .
$$

Using this expression for $E^{2}$, (B.6a) is readily inferred from Proposition B.3 and (B.6d), which is established below. Equation (B.6b) is a direct consequence of the definition of $E^{1}$ and the fact that $P_{\lambda}$ is homogeneous of degree $|\lambda|$. We observe that

$$
t_{1}\left(P_{\lambda}\right)=P_{\lambda}+E^{0} P_{\lambda}+\text { l.d. }
$$

(where l.d. stands for terms of lower degree); c.f., (4.20) for $\gamma=1$. Inserting this expression into the left-hand side of (B.4), and then comparing coefficients with the right-hand side, yields (B.6c).

It is clear from the discussion in Section 2.3 and Lemma 2.4 that $D:=D^{2}-(2 / \alpha)\left(p_{0}-1\right) E^{1}$ is the inverse limit of $D_{n}$, as defined in (2.7). Consulting Example 3 in Section VI.4 of Macdonald [29], we thus conclude that $P_{\lambda}$ is an eigenfunction of $D$ with eigenvalue $\sum_{i=1}^{\ell(\lambda)} \lambda_{i}\left(\lambda_{i}-1-\right.$ $2(i-1) / \alpha)$. Hence, (B.6d) follows from (B.6b). The remaining two equations (B.6e), (B.6f) can now be deduced by a direct computation using (B.1b). 
Remark B.5. Combining the relations in Lemma B.1 with Pieri formulae for Jack's symmetric functions, we could, in principle, compute the action of the differential operators $E^{\ell}$ or $D^{k}$ for any $\ell, k \in \mathbb{N}$. However, the the resulting formulae become more and more complex as the values of $\ell$ and $k$ are increased. Since we shall only make use of the formulae obtained in Lemma B.4, we therefore refrain from any further such computations.

As a direct application of Lemma B.4, we have the following theorem:

Theorem B.6. In (B.2) fix $a_{k}, b_{\ell} \in \mathbf{F}$ such that $a_{2}$ and $b_{1}$ are not both zero. Then, for any partition $\lambda$, there exists a unique symmetric function $F_{\lambda} \in \Lambda_{\mathbf{F}}$ such that

1. $F_{\lambda}=P_{\lambda}+\sum_{\mu \subset \lambda} u_{\lambda \mu} P_{\lambda}$ for some $u_{\lambda \mu} \in \mathbf{F}$ (triangularity);

2. $\mathcal{L} F_{\lambda}=\varepsilon_{\lambda} F_{\lambda}$ for some $\epsilon_{\lambda} \in \mathbf{F}$ (eigenfunction);

Moreover, the eigenvalue $\epsilon_{\lambda}$ is given explicitly by

$$
\varepsilon_{\lambda}=a_{2} \sum_{i} \lambda_{i}\left(\lambda_{i}-1+\frac{2}{\alpha}\left(p_{0}-i\right)\right)+b_{1}|\lambda|
$$

Proof. According to Lemma B.4, we have that

$$
\mathcal{L}\left(P_{\lambda}\right)=\varepsilon_{\lambda} P_{\lambda}+\sum_{\mu \subset \lambda} c_{\lambda \mu} P_{\mu}, \quad \varepsilon_{\lambda}=a_{2} d_{\lambda}+b_{1}|\lambda|,
$$

for some coefficients $c_{\lambda \mu}$, and with $d_{\lambda}$ as specified in (B.6d). Furthermore, it follows from (2.1), (2.10) and (B.5) that $c_{\lambda \mu} \in \mathbf{F}$. Let us now make the ansatz $F_{\lambda}=\sum_{\mu \subseteq \lambda} u_{\lambda \mu} P_{\mu}$ with $u_{\lambda \lambda} \equiv 1$. By a direct computation, we then find that the eigenvalue equation $\mathcal{L} F_{\lambda}=\epsilon_{\lambda} F_{\lambda}$ holds true if and only if the coefficients $u_{\lambda \mu}$ satisfy the recurrence relation

$$
\left(\epsilon_{\lambda}-\epsilon_{\mu}\right) u_{\lambda \mu}=\sum_{\mu \subset \nu \subseteq \lambda} u_{\lambda \mu} c_{\nu \mu}
$$

We observe that

$$
\epsilon_{\lambda}-\epsilon_{\mu}=a_{2} \sum_{i}\left(\lambda_{i}^{2}-\mu_{i}^{2}\right)+\left(b_{1}-a_{2}\right)(|\lambda|-|\mu|)+\frac{2 a_{2}}{\alpha} \sum_{i}\left(\lambda_{i}-\mu_{i}\right)\left(p_{0}-i\right),
$$

which clearly is non-zero for all $\mu \subset \lambda$. Since we have fixed $u_{\lambda \lambda} \equiv 1$, this means that the coefficients $u_{\lambda \mu}$ are uniquely determined by the recurrence relation (B.8). Finally, the fact that $c_{\lambda \mu}, \epsilon_{\lambda} \in \mathbf{F}$ implies that also $u_{\lambda \mu} \in \mathbf{F}$.

Fix $m \in \mathbb{N}$, and let $\operatorname{Par}_{m}$ denote the set of partitions $\lambda$ of weight $|\lambda| \leq m$. It is clear from (2) in Theorem B.6 that there exists a transition matrix $\left(M_{\lambda \mu}\right)$ from the symmetric functions $\left\{F_{\lambda}\right\}_{\lambda \in \operatorname{Par}_{m}}$ to the set of Jack's symmetric functions $\left\{P_{\lambda}\right\}_{\lambda \in \operatorname{Par}_{m}}$, given by

$$
F_{\lambda}=\sum_{\mu} M_{\lambda \mu} P_{\mu}
$$

Fix a total order $<_{t}$ on $\operatorname{Par}_{m}$ that is compatible with the order given by inclusion of diagrams, i.e., if $\mu \subset \lambda$, then $\mu<_{t} \lambda$. If we order the entries of $\left(M_{\lambda \mu}\right)$ according to this total order, then Property (2) in Theorem B.6 implies that we obtain a lower triangular matrix with one's on the diagonal. Hence, $\left(M_{\lambda \mu}\right)$ is invertible, i.e., Jack's symmetric functions can be expressed as linear combinations of the $F_{\lambda}$. Since Jack's symmetric functions form a basis for $\Lambda_{\mathbb{F}}$, we thus arrive at the following corollary: 
Corollary B.7. Let $a_{k}$ and $b_{\ell}$ be as in Theorem B.6. Then, as $\lambda$ runs through the set of all partitions, the symmetric functions $F_{\lambda}$ form a basis for $\Lambda_{\mathbf{F}}$.

We note that the eigenfunctions in Theorem B.6 have the following useful representation:

$$
F_{\lambda}=\prod_{\mu \subset \lambda} \frac{\mathcal{L}-\varepsilon_{\mu}}{\varepsilon_{\lambda}-\varepsilon_{\mu}}\left(P_{\lambda}\right)
$$

To establish this formula, we first note that the triangularity property (1) can be directly inferred from Lemma B.4. In addition, from the proof of Theorem B.6 we recall the triangular action (B.7) of $\mathcal{L}$ on $P_{\lambda}$. It follows that the differential operator $\prod_{\mu \subset \lambda}\left(\mathcal{L}-\epsilon_{\mu}\right)$ annihilates the subspace spanned by Jack's symmetric functions $P_{\mu}$ with $\mu \subset \lambda$. Clearly, this fact implies the eigenfunction property (2).

Since we make use of it in Section 4, we also note the following lemma:

Lemma B.8. For $\ell, k \in \mathbb{N}_{0}$,

$$
t_{\gamma} \circ E^{\ell}=\left(\sum_{m=0}^{\ell} \gamma^{\ell-m}\left(\begin{array}{c}
\ell \\
m
\end{array}\right) E^{m}\right) \circ t_{\gamma}, \quad t_{\gamma} \circ D^{k}=\left(\sum_{m=0}^{k} \gamma^{k-m}\left(\begin{array}{c}
k \\
m
\end{array}\right) D^{m}\right) \circ t_{\gamma} .
$$

Proof. We first observe that the analogous statement for finitely many variables is easily verified. Fix $n \in \mathbb{N}$, and define a homomorphism $t_{\gamma, n}: \Lambda_{\mathbb{F}, n} \rightarrow \Lambda_{\mathbb{F}, n}$ by setting

$$
t_{\gamma, n}(p)\left(x_{1}, \ldots, x_{n}\right)=p\left(x_{1}+\gamma, \ldots, x_{n}+\gamma\right), \quad \forall p \in \Lambda_{\mathbb{F}, n} .
$$

We note the intertwining relation $\varphi_{n} \circ t_{\gamma}=t_{\gamma, n} \circ \varphi_{n}$; c.f., the discussion succeeding (4.20). Clearly, we have that

$$
t_{\gamma, n} \circ E_{n}^{\ell}=\left(\sum_{i=1}^{n}\left(x_{i}+\gamma\right)^{\ell} \frac{\partial}{\partial x_{i}}\right) \circ t_{\gamma, n}=\left(\sum_{m=0}^{\ell} \gamma^{\ell-m}\left(\begin{array}{c}
\ell \\
m
\end{array}\right) E_{n}^{m}\right) \circ t_{\gamma, n} .
$$

From Lemma 2.4 we thus infer that

$$
\begin{aligned}
\varphi_{n} \circ t_{\gamma} \circ E^{\ell} & =t_{\gamma, n} \circ E_{n}^{\ell} \circ \varphi_{n}=\left(\sum_{m=0}^{\ell} \gamma^{\ell-m}\left(\begin{array}{c}
\ell \\
m
\end{array}\right) E_{n}^{m}\right) \circ t_{\gamma, n} \circ \varphi_{n} \\
& =\varphi_{n} \circ\left(\sum_{m=0}^{\ell} \gamma^{\ell-m}\left(\begin{array}{c}
\ell \\
m
\end{array}\right) E^{m}\right) \circ t_{\gamma} .
\end{aligned}
$$

Finally the fact that this equation holds true for all $n \in \mathbb{N}$ implies the statement for $E^{\ell}$; c.f., the proof of Lemma 2.4. The differential operator $D^{k}$ can be treated similarly.

\section{Proof of Theorem 4.15}

The starting point is a sequence of recurrence relations for the Jacobi symmetric polynomials $\mathcal{J}_{\lambda}(x)$, as deduced by van Diejen [47] (see Theorem 6.4), which we now recall. In doing so, we shall essentially employ the formulation in Section 2 of Sergeev and Veselov [41].

Theorem C.1 (van Diejen [47]). For generic parameter values, the generalised Jacobi polynomials $\mathcal{J}_{\lambda}(x)$ satisfy the recurrence relations

$$
2^{r} e_{r}(x) \frac{\mathcal{J}_{\lambda}(x)}{\mathcal{J}_{\lambda}\left(0^{n}\right)}=\sum_{\epsilon(J), \epsilon(K)}(-1)^{|K|} \hat{V}_{I(n), \epsilon(J)}^{(+)}\left(\rho^{J}(n)+\lambda\right) \hat{V}_{J^{c}, \epsilon(K)}^{(-)}\left(\rho^{J}(n)+\lambda\right) \frac{\mathcal{J}_{\lambda+e_{\epsilon(J)}}(x)}{\mathcal{J}_{\lambda+e_{\epsilon(J)}}\left(0^{n}\right)},
$$

where the sum is over all sequences of signs $\epsilon(J)$ and $\epsilon(K)$ with $J, K \subseteq I(n) \equiv\{1, \ldots, n\}$ such that $J \cap K=\varnothing,|J|+|K|=r$, and $\lambda+e_{\epsilon(J)}$ is a partition. 
In order deduce the corresponding recurrence relations for the Jacobi symmetric functions it is important to know how (C.1) depends on the number of variables $n$. To this end, we first observe that the sum over $\epsilon(J)$ is limited by the requirement that $\lambda+e_{\epsilon(J)}$ should be a partition, and consequently does not depend in any essential way on $n$. However, there is no such obvious limitation on the sum over $\epsilon(K)$. In addition, it is not a priori clear how the coefficients in (C.1), as well as the specialization $\mathcal{J}_{\lambda}\left(0^{n}\right)$, depend on $n$. The first problem was resolved by Sergeev and Veselov [41] (see Lemma 4.1) through the following lemma:

Lemma C.2 (Sergeev and Veselov [41]). Let $J, K \subseteq I(m) \equiv\{1, \ldots, m\}$ for some positive integer $m \geq \ell(\lambda)$, and $\epsilon(J)$ a corresponding conf iguration of signs. If $\lambda+\epsilon(J)$ is a partition, and $\max (K) \geq \ell(\lambda)+|K|+1$ and $\max (K) \geq \max (J)+|K|+1$, then $\hat{V}_{J^{c}, \epsilon(K)}^{(-)}\left(\rho^{J}+\lambda\right)=0$.

Let $r=|J|+|K|$. In order for $\lambda+e_{\epsilon(J)}$ to be a partition, it is clear that we must have $\max (J) \leq \ell(\lambda)+|J|$. It follows that the inequalities in the Lemma are satisfied for all $K \subseteq I$ such that $\max (K) \geq \ell(\lambda)+r+1$. Hence, we can restrict our attention to $J, K \subseteq I(\ell(\lambda)+r)$ irrespective of the specific value of $n$.

We proceed to consider the second problem: the dependence of the coefficients $\hat{V}_{I, \epsilon(J)}^{(+)}\left(\rho^{J}+\lambda\right)$ and $\hat{V}_{J^{c}, \epsilon(K)}^{(-)}\left(\rho^{J}+\lambda\right)$ on $n$. We shall require a somewhat more detailed resolution of this problem than that stated by Sergeev and Veselov [41] in their Lemma 4.2.

Lemma C.3. Let $n \in \mathbb{N}$ be such that $n \geq \ell(\lambda)+r+1$. Then,

$$
\begin{aligned}
& \hat{V}_{I(n), \epsilon(J)}^{(+)}\left(\rho^{J}(n)+\lambda\right)=\hat{V}_{I(\ell(\lambda)+r), \epsilon(J)}^{(+)}\left(\rho^{J}(n)+\lambda\right) R_{\epsilon(J)}\left(\rho^{J}(n)+\lambda ; \ell(\lambda)+r\right), \\
& \hat{V}_{J^{c}, \epsilon(K)}^{(-)}\left(\rho^{J}(n)+\lambda\right)=\hat{V}_{I(\ell(\lambda)+r) \backslash J, \epsilon(K)}^{(-)}\left(\rho^{J}(n)+\lambda\right) R_{\epsilon(K)}\left(\rho^{J}(n)+\lambda ; \ell(\lambda)+r\right) .
\end{aligned}
$$

Proof. As previously observed, $\lambda+e_{\epsilon(J)}$ is a partition only if $J \subseteq I(\ell(\lambda)+r)$. It follows that

$$
I \backslash J=(I(\ell(\lambda)+r) \backslash J) \cup\{\ell(\lambda)+r+1, \ldots, n\} .
$$

We observe that, for any $m \in \mathbb{N}$ such that $\ell(\lambda)<m \leq n$,

$$
\begin{aligned}
& \prod_{j \in J} \prod_{i=m}^{n} \hat{v}^{J}\left(\epsilon_{j}(\rho(n)+\lambda)_{j}+(\rho(n)+\lambda)_{i}\right) \hat{v}^{J}\left(\epsilon_{j}(\rho(n)+\lambda)_{j}-(\rho(n)+\lambda)_{i}\right) \\
& \quad=R_{\epsilon(J)}(\rho(n)+\lambda ; m)
\end{aligned}
$$

which thus depends on $n$ only through $\rho(n)$. Using these facts, it is straightforward to infer equation (C.2a) from the explicit definition of the function $\hat{V}^{(+)}$in (4.23a). The validity of (C.2b) follows similarly once it is observed that, since also $K \subseteq I(\ell(\lambda)+r)$,

$$
J^{c} \backslash K=(I(\ell(\lambda)+r) \backslash J) \backslash K \cup\{\ell(\lambda)+r+1, \ldots, n\} .
$$

We recall that a formula for the specialisation of $\mathcal{J}_{\lambda}(x)$ at $x=0^{n}$ can be obtained from Corollary 5.2 in Opdam [36] by specialising to the root system $B C_{n}$ (and taking into account the relation between the $\mathcal{J}_{\lambda}$ and the multivariable Jacobi polynomials considered by Opdam; see Beerends and Opdam [3] and Sergeev and Veselov [41]). Although his formula does not directly generalise to the Jacobi symmetric functions, Sergeev and Veselov [41] (see Proposition 2.3) showed that it is given by the right hand side of $(4.22)$ for $p_{0}=n$. If we combine this observation with the two Lemmas above, then we can rewrite van Diejen's recurrence relations (C.1) in the form (4.24) for $p_{0}=n$. Since the sum does not depend on $n$, and the coefficients are rational functions of $n$, the validity of Theorem 4.15 follows. 


\section{Acknowledgements}

The work of P.D. was supported by FONDECYT grant \#1090034 and by CONICYT through the Anillos de Investigación RED4 y ACT56. M.H. would like to thank O. Chalykh, S.N.M. Ruijsenaars and A.P. Veselov for helpful discussions.

\section{References}

[1] Atiyah M.F., Macdonald I.G., Introduction to commutative algebra, Addison-Wesley Publishing Co., 1969.

[2] Baker T.H., Forrester P.J., The Calogero-Sutherland model and generalized classical polynomials, Comm. Math. Phys. 188 (1997), 175-216, solv-int/9608004.

[3] Beerends R.J., Opdam E.M., Certain hypergeometric series related to the root system BC, Trans. Amer. Math. Soc. 339 (1993), 581-609.

[4] Bernard D., Gaudin M., Haldane F.D.M., Pasquier V., Yang-Baxter equation in long-range interacting systems, J. Phys. A: Math. Gen. 26 (1993), 5219-5236, hep-th/9301084.

[5] Calogero F., Solution of the one-dimensional $N$-body problems with quadratic and/or inversely quadratic pair potentials, J. Math. Phys. 12 (1971), 419-436.

[6] Chalykh O., Feigin M., Veselov A., New integrable generalizations of Calogero-Moser quantum problem, J. Math. Phys. 39 (1998), 695-703.

[7] Cherednik I., Integration of quantum many-body problems by affine Knizhnik-Zamolodchikov equations, Adv. Math. 106 (1994), 65-95.

[8] Constantine A.G., The distribution of Hotelling's generalized $T_{0}^{2}$, Ann. Math. Statist. 37 (1966), $215-225$.

[9] Debiard A., Système différentiel hypergéométrique et parties radiales des opérateurs invariants des espaces symétriques de type $B C_{p}$, in Séminaire d'algèbre Paul Dubreil et Marie-Paule Malliavin (Paris, 1986), Lecture Notes in Math., Vol. 1296, Springer, Berlin, 1987, 42-124.

[10] Desrosiers P., Dang-Zheng L., Selberg integrals, super hypergeometric functions and applications to $\beta$ ensembles of random matrices, arXiv:1109.4659.

[11] Feigin M., Generalized Calogero-Moser systems from rational Cherednik algebras, Selecta Math. (N.S.) 18 (2012), 253-281, arXiv:0809.3487.

[12] Hallnäs M., Langmann E., A unified construction of generalized classical polynomials associated with operators of Calogero-Sutherland type, Constr. Approx. 31 (2010), 309-342, math-ph/0703090.

[13] Heckman G.J., Opdam E.M., Root systems and hypergeometric functions. I, Compositio Math. 64 (1987), $329-352$.

[14] Herz C.S., Bessel functions of matrix argument, Ann. of Math. (2) 61 (1955), 474-523.

[15] Heyneman R.G., Sweedler M.E., Affine Hopf algebras. I, J. Algebra 13 (1969), 192-241.

[16] James A.T., Special functions of matrix and single argument in statistics, in Theory and Application of Special Functions (Proc. Advanced Sem., Math. Res. Center, Univ. Wisconsin, Madison, Wis., 1975), Math. Res. Center, Univ. Wisconsin, Publ. No. 35, Academic Press, New York, 1975, 497-520.

[17] James A.T., Constantine A.G., Generalized Jacobi polynomials as spherical functions of the Grassmann manifold, Proc. London Math. Soc. (3) 29 (1974), 174-192.

[18] Kaneko J., Selberg integrals and hypergeometric functions associated with Jack polynomials, SIAM J. Math. Anal. 24 (1993), 1086-1110.

[19] Kerov S., Okounkov A., Olshanski G., The boundary of the Young graph with Jack edge multiplicities, Int. Math. Res. Not. 1998 (1998), no. 4, 173-199.

[20] Koekoek R., Swarttouw R.F., The Askey-scheme of hypergeometric orthogonal polynomials and its $q-$ analogue, Report 98-17, Faculty of Technical Mathematics and Informatics, Delft University of Technology, 1998, http://aw.twi.tudelft.nl/ koekoek/askey/.

[21] Kohler H., Guhr T., Supersymmetric extensions of Calogero-Moser-Sutherland-like models: construction and some solutions, J. Phys. A: Math. Gen. 38 (2005), 9891-9915, math-ph/0510039.

[22] Korányi A., Hua-type integrals, hypergeometric functions and symmetric polynomials, in International Symposium in Memory of Hua Loo Keng, Vol. II (Beijing, 1988), Springer, Berlin, 1991, 169-180. 
[23] Lassalle M., Coefficients binomiaux généralisés et polynômes de Macdonald, J. Funct. Anal. 158 (1998), 289-324.

[24] Lassalle M., Polynômes de Hermite généralisés, C. R. Acad. Sci. Paris Sér. I Math. 313 (1991), 579-582.

[25] Lassalle M., Polynômes de Jacobi généralisés, C. R. Acad. Sci. Paris Sér. I Math. 312 (1991), 425-428.

[26] Lassalle M., Polynômes de Laguerre généralisés, C. R. Acad. Sci. Paris Sér. I Math. 312 (1991), $725-728$.

[27] Lassalle M., Une formule du binôme généralisée pour les polynômes de Jack, C. R. Acad. Sci. Paris Sér. I Math. 310 (1990), 253-256.

[28] Macdonald I.G., Hypergeometric functions, unpublished.

[29] Macdonald I.G., Symmetric functions and Hall polynomials, 2nd ed., Oxford Mathematical Monographs, The Clarendon Press, Oxford University Press, New York, 1995.

[30] Moens E.M., Van der Jeugt J., On dimension formulas for $\mathfrak{g l}(m \mid n)$ representations, J. Lie Theory 14 (2004), $523-535$.

[31] Muirhead R.J., Aspects of multivariate statistical theory, Wiley Series in Probability and Mathematical Statistics, John Wiley \& Sons Inc., New York, 1982.

[32] Okounkov A., Olshanski G., Shifted Jack polynomials, binomial formula, and applications, Math. Res. Lett. 4 (1997), 69-78, q-alg/9608020.

[33] Olshanetsky M.A., Perelomov A.M., Quantum integrable systems related to Lie algebras, Phys. Rep. 94 (1983), 313-404.

[34] Olshanski G., Laguerre and Meixner orthogonal bases in the algebra of symmetric functions, Int. Math. Res. Not., to appear, arXiv:1103.5848.

[35] Olshanski G., Laguerre and Meixner symmetric functions, and infinite-dimensional diffusion processes, J. Math. Sci. 174 (2011), 41-57, arXiv:1009.2037.

[36] Opdam E.M., Some applications of hypergeometric shift operators, Invent. Math. 98 (1989), 1-18.

[37] Opdam E.M., Lecture notes on Dunkl operators for real and complex reflection groups, MSJ Memoirs, Vol. 8, Mathematical Society of Japan, Tokyo, 2000.

[38] Rains E.M., BC ${ }_{n}$-symmetric polynomials, Transform. Groups 10 (2005), 63-132, math.QA/0112035.

[39] Reed M., Simon B., Methods of modern mathematical physics. I. Functional analysis, 2nd ed., Academic Press Inc., New York, 1980.

[40] Sergeev A., Superanalogs of the Calogero operators and Jack polynomials, J. Nonlinear Math. Phys. 8 (2001), 59-64, math.RT/0106222.

[41] Sergeev A.N., Veselov A.P., $B C_{\infty}$ Calogero-Moser operator and super Jacobi polynomials, Adv. Math. 222 (2009), 1687-1726, arXiv:0807.3858.

[42] Sergeev A.N., Veselov A.P., Deformed quantum Calogero-Moser problems and Lie superalgebras, Comm. Math. Phys. 245 (2004), 249-278, math-ph/0303025.

[43] Sergeev A.N., Veselov A.P., Generalised discriminants, deformed Calogero-Moser-Sutherland operators and super-Jack polynomials, Adv. Math. 192 (2005), 341-375, math-ph/0307036.

[44] Sergeev A.N., Veselov A.P., Quantum Calogero-Moser systems: a view from infinity, in XVIth International Congress on Mathematical Physics, World Sci. Publ., Hackensack, NJ, 2010, 333-337, arXiv:0910.5463.

[45] Stanley R.P., Some combinatorial properties of Jack symmetric functions, Adv. Math. 77 (1989), $76-115$.

[46] van Diejen J.F., Confluent hypergeometric orthogonal polynomials related to the rational quantum Calogero system with harmonic confinement, Comm. Math. Phys. 188 (1997), 467-497, q-alg/9609032.

[47] van Diejen J.F., Properties of some families of hypergeometric orthogonal polynomials in several variables, Trans. Amer. Math. Soc. 351 (1999), 233-270, q-alg/9604004.

[48] Yan Z.M., A class of generalized hypergeometric functions in several variables, Canad. J. Math. 44 (1992), $1317-1338$. 